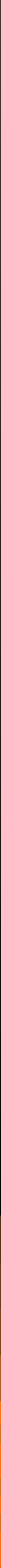




\section{Algebra \& Number Theory}

www.jant.org

\section{EDITORS}

\author{
MANAGING EDITOR \\ Bjorn Poonen \\ University of California \\ Berkeley, USA
}

\author{
EDITORIAL BOARD CHAIR \\ David Eisenbud \\ University of California \\ Berkeley, USA
}

\section{BOARD OF EDITORS}

Georgia Benkart

Dave Benson

Richard E. Borcherds

John H. Coates

J-L. Colliot-Thélène

Brian D. Conrad

Hélène Esnault

Hubert Flenner

Edward Frenkel

Andrew Granville

Joseph Gubeladze

Ehud Hrushovski

Craig Huneke

Mikhail Kapranov

Yujiro Kawamata

János Kollár

Hendrik W. Lenstra

Yuri Manin

Barry Mazur
University of Wisconsin, Madison, USA

University of Aberdeen, Scotland

University of California, Berkeley, USA

University of Cambridge, UK

CNRS, Université Paris-Sud, France

University of Michigan, USA

Universität Duisburg-Essen, Germany

Ruhr-Universität, Germany

University of California, Berkeley, USA

Université de Montréal, Canada

San Francisco State University, USA

Hebrew University, Israel

University of Kansas, USA

Yale University, USA

University of Tokyo, Japan

Princeton University, USA

Universiteit Leiden, The Netherlands

Northwestern University, USA

Harvard University, USA
Susan Montgomery

Shigefumi Mori

Andrei Okounkov

Raman Parimala

Victor Reiner

Karl Rubin

Peter Sarnak

Michael Singer

Ronald Solomon

Vasudevan Srinivas

J. Toby Stafford

Bernd Sturmfels

Richard Taylor

Ravi Vakil

Michel van den Bergh

Marie-France Vignéras

Kei-Ichi Watanabe

Andrei Zelevinsky

Efim Zelmanov
University of Southern California, USA

RIMS, Kyoto University, Japan

Princeton University, USA

Emory University, USA

University of Minnesota, USA

University of California, Irvine, USA

Princeton University, USA

North Carolina State University, USA

Ohio State University, USA

Tata Inst. of Fund. Research, India

University of Michigan, USA

University of California, Berkeley, USA

Harvard University, USA

Stanford University, USA

Hasselt University, Belgium

Université Paris VII, France

Nihon University, Japan

Northeastern University, USA

University of California, San Diego, USA

\section{PRODUCTION}

ant@mathscipub.org

Paulo Ney de Souza, Production Manager

Silvio Levy, Senior Production Editor

See inside back cover or www.jant.org for submission instructions.

Regular subscription rate for 2007: $\$ 180.00$ a year (\$120.00 electronic only).

Subscriptions, requests for back issues from the last three years and changes of subscribers address should be sent to Mathematical Sciences Publishers, Department of Mathematics, University of California, Berkeley, CA 94720-3840, USA.

Algebra \& Number Theory, ISSN 1937-0652, at Mathematical Sciences Publishers, Department of Mathematics, University of California, Berkeley, CA 94720-3840 is published continuously online. Periodical rate postage paid at Berkeley, CA 94704, and additional mailing offices.

\section{PUBLISHED BY \\ - mathematical sciences publishers \\ http://www.mathscipub.org \\ A NON-PROFIT CORPORATION \\ Typeset in LATEX}

Copyright $\odot 2008$ by Mathematical Sciences Publishers 


\title{
Contracted ideals and the Gröbner fan of the rational normal curve
}

\author{
Aldo Conca, Emanuela De Negri and Maria Evelina Rossi
}

\begin{abstract}
The paper has two goals: the study of the associated graded ring of contracted homogeneous ideals in $K[x, y]$ and the study of the Gröbner fan of the ideal $P$ of the rational normal curve in $\mathbb{P}^{d}$. These two problems are, quite surprisingly, very tightly related. We completely classify the contracted ideals with CohenMacaulay associated graded ring in terms of the numerical invariants arising from Zariski's factorization. We determine explicitly the initial ideals (monomial or not) of $P$, that are Cohen-Macaulay.
\end{abstract}

\section{Introduction}

The goal of the paper is twofold:

(1) to describe the Cohen-Macaulay initial ideals of the defining ideal $P$ of the rational normal curve in $\mathbb{P}^{d}$ in its standard coordinate system and for every positive integer $d$, and

(2) to identify the homogeneous contracted ideals in $K[x, y]$ whose associated graded ring is Cohen-Macaulay.

The two problems are closely related. Indeed they are essentially equivalent, as we proceed to explain. Let $K$ be a field, $R=K[x, y]$ and $I$ be a homogeneous ideal of $R$ with $\sqrt{I}=\mathbf{m}=(x, y)$. Denote by $\operatorname{gr}_{I}(R)$ the associated graded ring

$$
\bigoplus_{k} I^{k} / I^{k+1}
$$

of $I$. The ideal $I$ is said to be contracted if it is contracted from a quadratic extension, that is, if there exists a linear form $z$ in $R$ such that

$$
I=I R[\mathbf{m} / z] \cap R .
$$

Contracted ideals have been introduced by Zariski in his studies on the factorization property of integrally closed ideals; see [Zariski and Samuel 1960, App. 5]

MSC2000: primary 13A30; secondary 13P10, 13D40.

Keywords: Gröbner fan, contracted ideal, Rees algebra, rational normal curve, Cohen-Macaulay ring. 
or [Huneke and Swanson 2006, Chap. 14]. Every integrally closed ideal $I$ is contracted and has a Cohen-Macaulay associated graded $\operatorname{ring} \operatorname{gr}_{I}(R)$; see [Lipman and Teissier 1981; Huneke 1989]. On the contrary, the associated graded ring of a contracted ideal does not need to be Cohen-Macaulay. Zariski proved a factorization theorem for contracted ideals, asserting that every contracted ideal $I$ has a factorization $I=L_{1} \cdots L_{s}$ where $L_{i}$ are themselves contracted but of a very special kind. In the homogeneous case and with $K$ assumed to be algebraically closed, each $L_{i}$ is a lex-segment monomial ideal in a specific coordinate system depending on $i$. Recall that a monomial ideal $L$ in $R$ is a lex-segment ideal if that $x^{a} y^{b} \in L$ with $b>0$ implies that $x^{a+1} y^{b-1} \in L$ also. In [Conca et al. 2005, Corollary 3.14] it is shown that the Cohen-Macaulayness of $\operatorname{gr}_{I}(R)$ is equivalent to the CohenMacaulayness of $\operatorname{gr}_{L_{i}}(R)$ for every $i=1, \ldots, s$. Therefore to answer (2) one has to characterize the lex-segment ideals $L$ with Cohen-Macaulay associated graded ring. Any lex-segment ideal $L$ of initial degree $d$ can be encoded by a vector $a=\left(a_{0}, a_{1}, \ldots, a_{d}\right)$ with increasing integral coordinates and $a_{0}=0$. Given $L$ associated to $a$, we show that $\operatorname{gr}_{L}(R)$ is Cohen-Macaulay if and only if $\operatorname{in}_{a}(P)$ defines a Cohen-Macaulay ring. Here $\operatorname{in}_{a}(P)$ denotes the ideal of the initial forms of $P$ with respect to the vector $a$. Therefore (1) and (2) are indeed equivalent problems. In Section 4 we solve problem (1) by showing first that $P$ has exactly $2^{d-1}$ Cohen-Macaulay initial monomial ideals; see Theorem 4.11. Then we show that every Cohen-Macaulay initial ideal of $P$ has a Cohen-Macaulay monomial initial ideal; see Theorem 4.13. In terms of the Gröbner fan of $P$, Theorem 4.13 can be rephrased as that $\operatorname{in}_{a}(P)$ is Cohen-Macaulay if and only if $a$ belongs to the union of $2^{d-1}$ maximal closed cones. These cones are explicitly described by linear homogeneous inequalities. The fact that $P$ has exactly $2^{d-1}$ Cohen-Macaulay monomial initial ideals can be derived by combining the results of Hoşten and Thomas [2003] and those of O'Shea and Thomas [2005]; see Remark 4.20.

In Section 5 we give an explicit characterization, in terms of the numerical invariants arising from Zariski's factorization, of the Cohen-Macaulay property of the associated graded ring of a contracted homogeneous ideal in $K[x, y]$. In Section 6 we describe the relationship between the Hilbert series of $\operatorname{gr}_{L}(R)$ and the multigraded Hilbert series of $\operatorname{in}_{a}(P)$. We discuss also how the formulas for the Hilbert series and the polynomials of $\operatorname{gr}_{L}(R)$ change by varying the corresponding cones of the Gröbner fan of $P$. This has a conjectural relation with the hypergeometric Gröbner fan introduced by Saito, Sturmfels and Takayama in [Saito et al. 2000]. In Section 7 we show that the union of a certain subfamily of the $2^{d-1}$ Cohen-Macaulay cones is itself a cone. We call it the big Cohen-Macaulay cone. Indeed, the big Cohen-Macaulay cone is the union of $f_{d}$ Cohen-Macaulay cones of the Gröbner fan of $P$, where $f_{d}$ denotes the $(d+1)$-th Fibonacci number. In Section 8 we present some examples. 


\section{Notation and preliminaries}

Let $S$ be a polynomial ring over a field $K$ with maximal homogeneous ideal $\mathbf{m}$. For a homogeneous ideal $I$ of $S$ we denote by $\operatorname{gr}_{I}(S)$, Rees $(I)$ and $F(I)$ respectively the associated graded ring $\bigoplus_{k \in \mathbb{N}} I^{k} / I^{k+1}$, the Rees algebra $\bigoplus_{k \in \mathbb{N}} I^{k}$ and the fiber cone $\bigoplus_{k \in \mathbb{N}} I^{k} / \mathbf{m} I^{k}$ of $I$. By the very definition $F(I)$ is a standard graded $K$-algebra. Furthermore Rees $(I)$ can be identified with the $S$-subalgebra of the polynomial ring $S[t]$ generated by $f t$ with $f \in I$.

Let $I \subset S=K\left[x_{1}, \ldots, x_{n}\right]$ be a homogeneous ideal. We may consider the (standard) Hilbert function, Hilbert polynomial and Hilbert series of $S / I$. The Hilbert series of $S / I$ is $\sum_{i \geq 0} \operatorname{dim}_{K}(S / I)_{i} z^{i}$ and we denote it by $H_{S / I}(z)$. The series $H_{S / I}(z)$ has a rational expression $h(z) /(1-z)^{d}$ where $h(z) \in \mathbb{Z}[z]$ and $d$ is the Krull dimension of $S / I$. The polynomial $h(z)$ is called the (standard) $h$ polynomial of $S / I$. In particular, $h(0)=1$ and $h(1)$ is the ordinary multiplicity of $S / I$, denoted by $e(S / I)$.

If $I$ is $\mathbf{m}$-primary, we may consider also the (local) Hilbert functions, Hilbert polynomials and Hilbert series of $I$ (or of $\operatorname{gr}_{I}(S)$ ). There are two Hilbert functions associated with $I$ in this context. We denote them by $H(I, k)$ and $H^{1}(I, k)$ and they are defined by

$$
H(I, k)=\operatorname{dim}_{K}\left(I^{k} / I^{k+1}\right) \quad \text { and } \quad H^{1}(I, k)=\operatorname{dim}_{K}\left(S / I^{k+1}\right) .
$$

The corresponding Hilbert series are

$$
H_{I}(z)=\sum_{k \geq 0} H(I, k) z^{k} \quad \text { and } \quad H_{I}^{1}(z)=\sum_{k \geq 0} H^{1}(I, k) z^{k} .
$$

Obviously, $H_{I}(z)=(1-z) H_{I}^{1}(z)$. The series $H_{I}^{1}(z)$ has a rational expression

$$
H_{I}^{1}(z)=\frac{h(z)}{(1-z)^{n+1}}
$$

where $h(z)$ is a polynomial with integral coefficients and is called the (local) $h$ polynomial of $I$ or of $\operatorname{gr}_{I}(S)$. The Hilbert functions $H(I, k)$ and $H^{1}(I, k)$ agree for large $k$ with polynomials $P_{I}(z)$ and $P_{I}^{1}(z)$ at $z=k$. The polynomials $P_{I}(z)$ and $P_{I}^{1}(z)$ are called the Hilbert polynomials of $I$. Their coefficients, with respect to an appropriate binomial basis, are integers and are called Hilbert coefficients of $I$ and are denoted by $e_{i}(I)$. Precisely,

$$
P_{I}^{1}(z)=\sum_{i=0}^{n}(-1)^{i} e_{i}(I)\left(\begin{array}{c}
n-i+z \\
n-i
\end{array}\right) .
$$

In particular, $h(0)=\operatorname{dim}_{K} S / I$ and $h(1)=e_{0}(I)$ that is the multiplicity of $I$. 
Definition 2.1. Let $I \subset S=K\left[x_{1}, \ldots, x_{n}\right]$ be a homogeneous ideal of codimension $c$ and not containing linear forms. Then

(1) $S / I$ has minimal multiplicity if $e(S / I)=c+1$, and

(2) $S / I$ has a short $h$-vector if its $h$-polynomial is $1+c z$, that is, if the Hilbert series of $S / I$ is $(1+c z) /(1-z)^{n-c}$.

We denote the Castelnuovo-Mumford regularity of a graded $S$-module $M$ by $\operatorname{reg}(M)$. For results on the Castelnuovo-Mumford regularity and the minimal multiplicity we refer the readers to [Eisenbud and Goto 1984]. We just recall that if $S / I$ has a short $h$-vector, then it has minimal multiplicity. On the other hand, if $S / I$ is Cohen-Macaulay with minimal multiplicity, then it has a short $h$-vector. We will need the next lemma whose easy proof follows from the standard facts.

Lemma 2.2. Let I $\subset S$ be a homogeneous ideal. Assume $S / I$ has a short h-vector. Then $S / I$ is Cohen-Macaulay if and only if $\operatorname{reg}(I)=2$.

Every vector $a=\left(a_{0}, \ldots, a_{d}\right) \in \mathbb{Q}_{\geq 0}^{d+1}$ induces a graded structure on the polynomial ring $K\left[t_{0}, \ldots, t_{d}\right]$ by letting $\operatorname{deg} t_{i}=a_{i}$. Every monomial $t^{\alpha}$ is then homogeneous of degree

$$
\operatorname{deg}_{a} t^{\alpha}=a \alpha=\sum_{i=0}^{d} a_{i} \alpha_{i} .
$$

For every nonzero polynomial $f=\sum_{i=1}^{k} \lambda_{i} t^{\alpha_{i}}$ we set

$$
\operatorname{deg}_{a} f=\max \left\{a \alpha_{i}: \lambda_{i} \neq 0\right\} \quad \text { and } \quad \operatorname{in}_{a}(f)=\sum_{a \alpha_{i}=\operatorname{deg}_{a} f} \lambda_{i} t^{\alpha_{i}} .
$$

Then for every ideal $I$ one defines the initial ideal $\operatorname{in}_{a}(I)$ of $I$ with respect to $a$ to be

$$
\operatorname{in}_{a}(I)=\left(\operatorname{in}_{a}(f): f \in I, \quad f \neq 0\right) .
$$

Similarly, given a term order $\tau$, we denote by $\operatorname{in}_{\tau}(I)$ the ideal of the initial monomials of elements of $I$. Given $a \in \mathbb{Q}_{\geq 0}^{d+1}$ the term order defined by

$$
t^{\alpha} \geq t^{\beta} \text { if and only if } a \alpha>a \beta \text { or }\left(a \alpha=a \beta \text { and } t^{\alpha} \geq t^{\beta} \text { with respect to } \tau\right)
$$

is denoted by $\tau a$.

One easily shows that $\operatorname{in}_{\tau}\left(\operatorname{in}_{a}(I)\right)=\operatorname{in}_{\tau a}(I)$. Hence $\operatorname{in}_{a}(I)$ and $I$ have a common monomial initial ideal. This shows (1) of the following lemma.

Lemma 2.3. Let I be a homogeneous ideal with respect to the ordinary grading $\operatorname{deg} t_{i}=1$ and let $a \in \mathbb{Q}_{\geq 0}^{d+1}$. Then

(1) $S / I$ and $S / \operatorname{in}_{a}(I)$ have the same Hilbert function, and

(2) depth $S / \operatorname{in}_{a}(I) \leq \operatorname{depth} S / I$. 
Part (2) follows from the standard one-parameter flat family argument; for details see [Eisenbud 1995, Chap. 15] or [Bruns and Conca 2003].

Definition 2.4. Let $P$ be the ideal of the rational normal curve of $\mathbb{P}^{d}$ in its standard embedding. Namely, $P$ is the kernel of the $K$-algebra map

$$
S=K\left[t_{0}, t_{1}, \ldots, t_{d}\right] \rightarrow K[x, y]
$$

sending $t_{i}$ to $x^{d-i} y^{i}$.

The ideal $P$ is minimally generated by the 2 -minors of the matrix

$$
T_{d}=\left(\begin{array}{cccccc}
t_{0} & t_{1} & t_{2} & \ldots & \ldots & t_{d-1} \\
t_{1} & t_{2} & \ldots & \ldots & t_{d-1} & t_{d}
\end{array}\right)
$$

and it contains the binomials of the form $t_{i_{1}} t_{i_{2}} \cdots t_{i_{k}}-t_{j_{1}} t_{j_{2}} \cdots t_{j_{k}}$ with

$$
0 \leq i_{v}, j_{v} \leq d \quad \text { and } \quad i_{1}+i_{2}+\cdots+i_{k}=j_{1}+j_{2}+\cdots+j_{k} .
$$

The Hilbert series of $S / P$ is $(1+(d-1) z) /(1-z)^{2}$.

\section{Contracted ideals in dimension 2}

We briefly recall from [Zariski and Samuel 1960, App. 5], [Huneke and Swanson 2006, Chap. 14] and [Conca et al. 2005] a few facts about contracted ideals. As we deal only with homogeneous ideals, we will state the results in the graded setting.

Assume $K$ is an algebraically closed field. Let $R=K[x, y]$ and denote by $\mathbf{m}$ its maximal homogeneous ideal. An $\mathbf{m}$-primary homogeneous ideal $I$ of $R$ is said to be contracted if it is contracted from a quadratic extension, that is, if there exists a linear form $z \in R$ such that $I=I S \cap R$, where $S=R[\mathbf{m} / z]$. The property of being contracted can be described in several ways; for instance see [Conca et al. 2005, Prop. 3.3]. To a contracted ideal $I$ one associates a form, the characteristic form of $I$, defined as $\operatorname{GCD}\left(I_{d}\right)$ where $d$ is the initial degree of $I$. For our goals, it is important to recall the following definition and theorem.

Definition 3.1. Let $I$ be a homogeneous $\mathbf{m}$-primary ideal in $R$ and let $Q=I R_{\mathbf{m}}$. Let $J \subset R_{\mathbf{m}}$ be a minimal reduction of $Q$. The deviation of $I$ is the length of $Q^{2} / J Q$. It will be denoted by $V(I)$.

Theorem 3.2. Let I be a homogeneous $\mathbf{m}$-primary ideal in $R$. One has

(1) $\operatorname{gr}_{I}(R)$ is Cohen-Macaulay if and only if $V(I)=0$, and

(2) $V(I)=e_{0}(I)-\operatorname{dim}_{K}\left(R / I^{2}\right)+2 \operatorname{dim}_{K}(R / I)$.

See [Huckaba and Marley 1993, Prop. 2.6, Thm. A] for a proof of (1) and [Valla 1979, Lemma 1] for a proof of (2). Similar results are proved also in [Verma 1991]. 
We recall now Zariski's factorization theorem for contracted ideals and a related statement, [Conca et al. 2005, Cor. 3.14], concerning associated graded rings. In our setting they can be stated as follows.

Theorem 3.3. (1) Any contracted ideal I has a factorization $I=L_{1} \cdots L_{s}$ where $L_{i}$ are homogeneous $\mathbf{m}$-primary contracted ideals with characteristic form of type $\ell_{i}^{\alpha_{i}}$ for pairwise linearly independent linear forms $\ell_{1}, \ldots, \ell_{s}$.

(2) With respect to the factorization in (1) one has

$$
\operatorname{depth} \operatorname{gr}_{I}(R)=\min \left\{\operatorname{depth} \operatorname{gr}_{L_{i}}(R): i=1, \ldots, s\right\} .
$$

Lemma 3.4. The fiber cone $F(I)$ of a contracted ideal I has a short h-vector. Its Hilbert series is $(1+(d-1) z) /(1-z)^{2}$, where $d$ is the initial degree of $I$.

Proof. A contracted ideal of initial degree $d$ is minimally generated by $d+1$ elements and products of contracted ideals are contracted. The initial degree of $I^{k}$ is $k d$. Hence $I^{k}$ has $k d+1$ minimal generators. It follows that the Hilbert series of $F(I)$ is $(1+(d-1) z) /(1-z)^{2}$.

A monomial m-primary ideal $I$ of $R=K[x, y]$ can be encoded in various ways. We use the following. Let $d \in \mathbb{N}$ be such that $x^{d} \in I$ and, for $i=0, \ldots, d$, set $a_{i}(I)=\min \left\{j: x^{d-i} y^{j} \in I\right\}$. Then we have $0=a_{0}(I) \leq a_{1}(I) \leq \cdots \leq a_{d}(I)$. Obviously, the map

$$
I \rightarrow a=\left(a_{0}(I), \ldots, a_{d}(I)\right)
$$

establishes a bijective correspondence between the set of $\mathbf{m}$-primary monomial ideals containing $x^{d}$ and the set of weakly increasing sequences $a=\left(a_{0}, \ldots, a_{d}\right)$ of nonnegative integers with $a_{0}=0$. The inverse map is

$$
a=\left(0=a_{0} \leq a_{1} \cdots \leq a_{d}\right) \rightarrow\left(x^{d-i} y^{a_{i}}: i=0, \ldots, d\right) .
$$

It is easy to see that if $a$ corresponds to $I$, then $\operatorname{dim}_{K} R / I=\sum_{i=0}^{d} a_{i}$. Furthermore, if $a^{\prime}$ corresponds to $J$, then the sequence associated to the product $I J$ is $\left(c_{0}, c_{1}, c_{2}, \ldots\right)$ where $c_{i}=\min \left\{a_{j}+a_{k}^{\prime}: j+k=i\right\}$. In particular:

Lemma 3.5. Let I be a monomial ideal and $a=\left(a_{0}, \ldots, a_{d}\right)$ be the corresponding sequence. Then the Hilbert function of I is given by

$$
H^{1}(I, k)=\sum_{i=0}^{(k+1) d} \min \left\{a_{j_{1}}+\cdots+a_{j_{k+1}}: j_{1}+\cdots+j_{k+1}=i\right\} .
$$

We set $b_{i}(I)=a_{i}(I)-a_{i-1}(I)$ for $i=1, \ldots, d$ and observe that the ideal $I$ can be as well described via the sequence $b_{1}(I), \ldots, b_{d}(I)$ of nonnegative integers.

A monomial ideal $I$ is a lex-segment ideal if $x^{i} y^{j} \in I$ for some $j>0$ implies $x^{i+1} y^{j-1} \in I$. The $\mathbf{m}$-primary lex-segment ideals are contracted and correspond 
exactly to strictly increasing $a$-sequences (equivalently, positive $b$-sequences) in the above correspondence, provided one takes $d=\min \left\{j \in \mathbb{N}: x^{j} \in I\right\}$.

Remark 3.6. With respect to suitable coordinate systems the ideals $L_{i}$ in Theorem 3.3 are lex-segment ideals.

It follows from Theorem 3.3 and Remark 3.6 that the study of the depth of the associated graded ring of contracted ideals boils down to the study of the depth of $\operatorname{gr}_{L}(R)$ for a lex-segment ideal $L$. One has depth $\operatorname{gr}_{L}(R)=\operatorname{depth} \operatorname{Rees}(L)-1$ since $R$ is regular; see [Huckaba and Marley 1993, Cor. 2.7]. Therefore we can as well study the depth of Rees $(L)$ for lex-segment ideals $L$. Trung and Hoa gave in [Trung and Hoa 1986] a characterization of the Cohen-Macaulay property of affine semigroup rings. Since $\operatorname{Rees}(L)$ is an affine semigroup ring one could hope to use their results to describe the lex-segment ideals $L$ such that $\operatorname{Rees}(L)$ is CohenMacaulay. In practice, however, we have not been able to follow this idea.

Let $L$ be the lex-segment ideal with the associated $a$-sequence $a=\left(a_{0}, \ldots, a_{d}\right)$ and $b$-sequence $\left(b_{1}, \ldots, b_{d}\right)$. We present $\operatorname{Rees}(L)$ as a quotient of $R\left[t_{0}, \ldots, t_{d}\right]$ by the $R$-algebra map

$$
\psi: R\left[t_{0}, \ldots, t_{d}\right] \rightarrow \operatorname{Rees}(L) \subset R[t]
$$

obtained by sending $t_{i} \mapsto x^{d-i} y^{a_{i}} t$. Set

$$
\mathbf{1}=(1,1, \ldots, 1) \in \mathbb{N}^{d+1}, \quad \text { and } \quad \mathbf{d}=(d, d-1, d-2, \ldots, 0) \in \mathbb{N}^{d+1} .
$$

Lemma 3.7. With the above notation, $\operatorname{ker} \psi$ is generated by the binomials

(1) $x t_{i}-y^{b_{i}} t_{i-1}$ with $i=1, \ldots, d$, and

(2) $t^{\alpha}-y^{u} t^{\beta}$ where $\alpha, \beta \in \mathbb{N}^{d+1}$ satisfy

$$
\mathbf{1}(\alpha-\beta)=0, \quad \mathbf{d}(\alpha-\beta)=0, \quad u=a(\alpha-\beta) \geq 0 .
$$

Proof. Let $J$ be the ideal generated by the binomials of type (1) and (2). Obviously $J \subseteq \operatorname{ker} \psi$. Since ker $\psi$ is generated by the binomials it contains, it is enough to show that every binomial $M_{1}-M_{2} \in \operatorname{ker} \psi$ with $\operatorname{GCD}\left(M_{1}, M_{2}\right)=1$ belongs to $J$. Up to multiples of elements of type (1), we may assume that if $x$ divides one of the $M_{i}$, say $M_{1}$, then $M_{1}=x^{i} y^{j} t_{0}^{k}$. But this clearly contradicts the fact that $M_{1}-M_{2} \in \operatorname{ker} \psi$. In other words, every binomial in ker $\psi$ is, up to multiples of elements of type (1), a multiple of an element of type (2).

Lemma 3.8. Let $L$ be a lex-segment ideal and $a=\left(a_{0}, \ldots, a_{d}\right)$ its associated a-sequence, then we have

(1) $\operatorname{Rees}(L) /(y) \operatorname{Rees}(L)=K\left[x, t_{0}, \ldots, t_{d}\right] / x\left(t_{1}, \ldots, t_{d}\right)+\operatorname{in}_{a}(P)$,

(2) $F(L)=K\left[t_{0}, \ldots, t_{d}\right] / \operatorname{in}_{a}(P)$, and 
(3) $\operatorname{depth} \operatorname{gr}_{L}(R)=\operatorname{depth} \operatorname{Rees}(L)-1=\operatorname{depth} F(L)$,

where $P$ is the ideal introduced in Definition 2.4.

Proof. Set $F=F(L), G=\operatorname{gr}_{L}(R)$ and $\mathscr{R}=\operatorname{Rees}(L)$. First note that (2) follows from (1) since $F=\mathscr{R} /(x, y) \mathscr{R}$. To prove (1) we have to show that

$$
\operatorname{ker} \psi+(y)=x\left(t_{1}, \ldots, t_{d}\right)+\operatorname{in}_{a}(P)+(y) .
$$

For the inclusion $\subseteq$ we show that the generators of ker $\psi$ of type (1) and (2) in Lemma 3.7 belong to the ideal on the right hand side. This is obvious for those of type (1). For those of type (2), note that for any such $t^{\alpha}-y^{u} t^{\beta}$ one has $t^{\alpha}-t^{\beta} \in P$ and $\operatorname{in}_{a}\left(t^{\alpha}-t^{\beta}\right)=t^{\alpha}$ if $u>0$ and $\operatorname{in}_{a}\left(t^{\alpha}-t^{\beta}\right)=t^{\alpha}-t^{\beta}$ if $u=0$.

The inclusion $\supseteq$ for the elements of $x\left(t_{1}, \ldots, t_{d}\right)$ is obvious. Further, since $P$ is generated by binomials, one knows that $\operatorname{in}_{a}(P)$ is generated by $\operatorname{in}_{a}\left(t^{\alpha}-t^{\beta}\right)$ with $t^{\alpha}-t^{\beta} \in P$; see [Sturmfels 1996, Chap. 1]. If $a(\alpha-\beta)=0$, then $\operatorname{in}_{a}\left(t^{\alpha}-t^{\beta}\right)=$ $t^{\alpha}-t^{\beta}$ and $t^{\alpha}-t^{\beta} \in \operatorname{ker} \psi$. If instead $u=a(\alpha-\beta)>0$, then $\operatorname{in}_{a}\left(t^{\alpha}-t^{\beta}\right)=t^{\alpha}$ and $t^{\alpha}-y^{u} t^{\beta} \in \operatorname{ker} \psi$ so $t^{\alpha} \in \operatorname{ker} \psi+(y)$.

To prove (3) note that $P \subseteq\left(t_{1}, \ldots, t_{d}\right)$ and hence $\operatorname{in}_{a}(P) \subseteq\left(t_{1}, \ldots, t_{d}\right)$. It follows that

$$
\left(t_{1}, \ldots, t_{d}\right) \subseteq\left(x\left(t_{1}, \ldots, t_{d}\right)+\operatorname{in}_{a}(P)\right): x \subseteq\left(t_{1}, \ldots, t_{d}\right): x=\left(t_{1}, \ldots, t_{d}\right) .
$$

Hence

$$
\left(t_{1}, \ldots, t_{d}\right)=\left(x\left(t_{1}, \ldots, t_{d}\right)+\operatorname{in}_{a}(P)\right): x
$$

and we get a short exact sequence

$$
0 \rightarrow K\left[x, t_{0}\right](-1) \rightarrow \mathscr{R} /(y) \mathscr{R} \rightarrow F \rightarrow 0 .
$$

By Lemma 3.4 the ring $F$ is 2-dimensional with short $h$-vector. It follows that the same is true for $\mathscr{R} /(y) \mathscr{R}$ with respect to the standard grading. Using the depth formula for short exact sequences [Bruns and Herzog 1993, Prop. 1.2.9], we have that if depth $\mathscr{R} /(y) \mathscr{R}$ is 0 or 1 , then depth $F=\operatorname{depth} \mathscr{R} /(y) \mathscr{R}$. Finally, if $\mathscr{R} /(y) \mathscr{R}$ is Cohen-Macaulay then $\operatorname{reg}(\mathscr{R} /(y) \mathscr{R})=1$ and it follows that $\operatorname{reg} F=1$. Then from Lemma 2.2 we can conclude that $F$ is Cohen-Macaulay.

We have shown that depth $\mathscr{R}-1=\operatorname{depth} \mathscr{R} /(y) \mathscr{R}=\operatorname{depth} F$. Since by [Huckaba and Marley 1993, Cor. 2.7] depth $G=\operatorname{depth} \mathscr{R}-1$, the proof of (3) is complete.

Summing up, we have shown:

Proposition 3.9. Let $L$ be a lex-segment ideal in $R=K[x, y]$ with associated a-sequence $a=\left(a_{0}, \ldots, a_{d}\right)$. Then

$$
\text { depth } \operatorname{gr}_{L}(R)=\operatorname{depth} K\left[t_{0}, \ldots, t_{d}\right] / \operatorname{in}_{a}(P)
$$

where $P$ is the ideal in Definition 2.4. 


\section{Cohen-Macaulay initial ideals of the ideal of the rational normal curve}

The results of the previous sections show that the study of the contracted ideals of $K[x, y]$ whose associated graded ring is Cohen-Macaulay is equivalent to the study of the initial ideals of $P$ defining Cohen-Macaulay rings. In this section we describe the initial ideals of $P$ (with respect to vectors and in the given coordinates) defining Cohen-Macaulay rings. We will say that an ideal $I$ is Cohen-Macaulay if the quotient ring defined by $I$ is Cohen-Macaulay. The steps of the classification are

(1) to classify the 2-dimensional Cohen-Macaulay monomial ideals with minimal multiplicity,

(2) to identify those of the form $\operatorname{in}_{\tau}(P)$ for some term order $\tau$ among the ideals of (1), and

(3) to identify the vectors $a \in \mathbb{Q}_{\geq 0}^{d+1}$ such that $\operatorname{in}_{a}(P)$ is Cohen-Macaulay (monomial or not).

We start by classifying the 2-dimensional Cohen-Macaulay square-free monomial ideals with minimal multiplicity. Square-free monomial ideals are in bijective correspondence with simplicial complexes. In particular, square-free monomial ideals defining algebras of Krull dimension 2 are in bijective correspondence with simplicial complexes of dimension 1, that is, graphs. The correspondence goes like this: to any graph with vertex set $V$ and edge set $E$ one associates the monomial ideal on variables $V$, whose generators are the products $x y$ such that $\{x, y\}$ is not in $E$ and the square-free monomials of degree 3.

Recall that a graph $G$ with $n$ vertices and $e$ edges is a tree if it satisfies the following equivalent conditions:

(1) For every distinct vertices $x$ and $y$, there exists exactly one path in $G$ connecting $x$ and $y$;

(2) $G$ is connected and $n-e=1$;

(3) $G$ is connected and if we remove any edge the resulting graph is disconnected.

Lemma 4.1. The 2-dimensional Cohen-Macaulay square-free monomial ideals with minimal multiplicity correspond to trees.

Proof. Let $G$ be a graph with $n$ vertices and $e$ edges. Let $A$ be the corresponding quotient ring. The Hilbert series of $A$ is given by

$$
1+\frac{n z}{(1-z)}+\frac{e z^{2}}{(1-z)^{2}}
$$


see [Bruns and Herzog 1993, 5.1.7]. This implies immediately that $A$ has minimal multiplicity if and only if $n-e=1$. The Cohen-Macaulay property of $A$ corresponds to the connectedness of $G$; see [Bruns and Herzog 1993, 5.1.26]. So we are considering connected graphs with $n-e=1$, that is, trees.

Next we extend our characterization from the square-free monomial ideals to general monomial ideals. We will make use of the following lemma, whose easy proof belongs to the folklore of the subject.

Lemma 4.2. Let I be a monomial ideal generated in degree 2 and with linear syzygies. Let $x, y, z$ be variables. We have

(1) if $x^{2}, y^{2} \in I$ then $x y \in I$, and

(2) if $x^{2}, y z \in I$ then either $x y \in I$ or $x z \in I$.

Proof. Say $I$ is generated by monomials $m_{1}=x^{2}, m_{2}=y^{2}$ and other monomials $m_{3}, \ldots, m_{s}$. Take a free module $F$ with basis $e_{1}, e_{2}, \ldots e_{s}$ and map $e_{j}$ to $m_{j}$. The syzygy module $\operatorname{Syz}(I)$ is generated by the reduced Koszul relations $a e_{i}-b e_{j}$ with $a=m_{j} / \operatorname{GCD}\left(m_{i}, m_{j}\right)$ and $b=m_{i} / \operatorname{GCD}\left(m_{i}, m_{j}\right)$. By assumption we know that $\operatorname{Syz}(I)$ is generated by the elements $a e_{i}-b e_{j}$ with $\operatorname{deg} a=1$. Call this set $G$. Now $y^{2} e_{1}-x^{2} e_{2}$ is in $\operatorname{Syz}(I)$ and therefore can be written as $\sum v_{i j}\left(a e_{i}-b e_{j}\right)$ where the sum is extended to the elements $a e_{i}-b e_{j} \in G$. It follows that there must be in $G$ an element of the form $y e_{1}-b e_{i}$. We deduce that $m_{i} / \operatorname{GCD}\left(m_{i}, x^{2}\right)=y$, forcing $m_{i}$ to be $x y$. Similarly one proves (2).

The crucial inductive step is encoded in the following lemma.

Lemma 4.3. Let $I \subset S=K\left[x_{1}, \ldots, x_{n}\right]$ be a monomial ideal such that $S / I$ is Cohen-Macaulay of dimension 2 with minimal multiplicity. Let $x_{i}$ be a variable such that $x_{i}^{2} \in I$. Set $S^{\prime}=K\left[x_{j}: 1 \leq j \leq n, j \neq i\right]$.

(1) $I:\left(x_{i}\right)$ is generated by exactly $n-2$ variables.

(2) Write $I+\left(x_{i}\right)=J+\left(x_{i}\right)$ where $J$ is a monomial ideal of $S^{\prime}$. Then $S^{\prime} / J$ is a 2-dimensional Cohen-Macaulay ring with minimal multiplicity.

Proof. First we show that $I:\left(x_{i}\right)$ is generated by variables. Let $m$ be one of the generators of $I$. We have to show that if $x_{i}$ does not divide $m$ then there exists a variable $x_{j}$ such that $x_{j} \mid m$ and $x_{i} x_{j} \in I$. Let $V$ be the set of the variables whose square is not in $I$ and let $Q$ be the set of the variables whose square is in $I$. If $m$ is divisible by a variable in $Q$, then we are done by Lemma 4.2 (1). Otherwise, if $m$ is not divisible by a variable in $Q$, then $m=x_{j} x_{k}$ with $x_{j}, x_{k} \in V, j \neq k$. By Lemma 4.2 (2) we have that either $x_{i} x_{j}$ or $x_{i} x_{k}$ is in $I$. Knowing that $I:\left(x_{i}\right)$ is generated by variables we deduce that $I:(x)$ is a prime ideal, hence an associated prime of the Cohen-Macaulay ideal $I$. Thus the codimension of $I:\left(x_{i}\right)$ is $n-2$. This proves (1). The standard short exact sequence $0 \rightarrow S / I:\left(x_{i}\right)(-1) \rightarrow S / I \rightarrow S^{\prime} / J \rightarrow 0$ shows 
that the Hilbert series of $S^{\prime} / J$ is $1+(n-3) z /(1-z)^{2}$. Hence $S^{\prime} / J$ has a short $h$-vector. Furthermore, the exact sequence implies that the regularity of $S^{\prime} / J$ is 1 , that is, $\operatorname{reg}(J)=2$. By Lemma 2.2 we conclude that $S^{\prime} / J$ is Cohen-Macaulay.

Corollary 4.4. With the assumptions in Lemma 4.3 and the notation of its proof, there exist $x_{j}, x_{k} \in V$ such that $I:\left(x_{i}\right)=\left(x_{v}: 1 \leq v \leq n\right.$ and $\left.v \notin\{j, k\}\right)$ and $x_{j} x_{k} \notin I$.

Proof. We know by Lemma 4.3 that there are variables $x_{j}$ and $x_{k}$ so that

$$
I:\left(x_{i}\right)=\left(x_{v}: 1 \leq v \leq n \text { and } v \notin\{j, k\}\right) .
$$

If $x_{j} x_{k} \in I$, then $x_{j} x_{k} \in I:\left(x_{i}\right)$. This is a contradiction.

Definition 4.5. Let $V$ and $Q$ be disjoint sets of variables. Let $G$ be a tree with vertex set $V$ and edge set $E$. Let $\phi: Q \rightarrow E$ be a map. Let $J$ be the square-free monomial ideal associated with $G$. Let $H=(Q)^{2}+(x y: x \in Q, y \in V$ and $y \notin \phi(x))$. We define

$$
I(G, \phi)=J+H .
$$

Example 4.6. Let $V=\left\{v_{1}, v_{2}, v_{3}, v_{4}\right\}$ and $Q=\left\{q_{1}, q_{2}, q_{3}, q_{4}, q_{5}\right\}$. Let $G$ be the tree on $V$ with edges $E=\left\{e_{1}, e_{2}, e_{3}\right\}$ where

$$
e_{1}=\left\{v_{1}, v_{2}\right\}, e_{2}=\left\{v_{1}, v_{3}\right\}, e_{3}=\left\{v_{3}, v_{4}\right\} .
$$

Let $\phi: Q \rightarrow E$ be the map sending $q_{1}, q_{2}, q_{3}, q_{4}, q_{5}$ to $e_{2}, e_{1}, e_{1}, e_{3}, e_{2}$, respectively. Then $J=\left(v_{1} v_{4}, v_{2} v_{3}, v_{2} v_{4}\right)$ and

$$
\begin{aligned}
H= & \left(q_{1}, q_{2}, q_{3}, q_{4}, q_{5}\right)^{2}+q_{1}\left(v_{2}, v_{4}\right)+q_{2}\left(v_{3}, v_{4}\right) \\
& +q_{3}\left(v_{3}, v_{4}\right)+q_{4}\left(v_{1}, v_{2}\right)+q_{5}\left(v_{2}, v_{4}\right) .
\end{aligned}
$$

In the next proposition we achieve the first step of the classification.

Proposition 4.7. Let I $\subset S$ be a monomial ideal. The following conditions are equivalent:

(1) there exist a tree $G$ and a map $\phi: Q \rightarrow E$ such that $I=I(G, \phi)$;

(2) $S / I$ is a 2-dimensional Cohen-Macaulay ring with minimal multiplicity.

Proof. First we show that every ideal of type $I(G, \phi)$ defines a Cohen-Macaulay, 2-dimensional ring with minimal multiplicity. We proceed by induction on the cardinality of $Q$. If $Q$ is empty, then the ideal $I$ is 2-dimensional Cohen-Macaulay with minimal multiplicity by Lemma 4.1. Now assume $Q$ is not empty and pick $q \in Q$. By construction $I(G, \phi):(q)$ is generated by $Q \cup V \backslash \phi(q)$ and

$$
I(G, \phi)+(q)=I\left(G, \phi^{\prime}\right)+(q)
$$


where $\phi^{\prime}$ is the restriction of $\phi$ to $Q^{\prime}=Q \backslash\{q\}$. By induction we know that $I\left(G, \phi^{\prime}\right) \subset K\left[V, Q^{\prime}\right]$ is 2-dimensional Cohen-Macaulay with minimal multiplicity. The short exact sequence

$$
0 \rightarrow K[V, Q] / I(G, \phi):(q)(-1) \rightarrow K[V, Q] / I(G, \phi) \rightarrow K\left[V, Q^{\prime}\right] / I\left(G, \phi^{\prime}\right) \rightarrow 0
$$

shows that $I(G, \phi)$ is 2-dimensional with minimal multiplicity and since both $K[V, Q] / I(G, \phi):(q)$ and $K\left[V, Q^{\prime}\right] / I\left(G, \phi^{\prime}\right)$ are 2-dimensional and CohenMacaulay, $K[V, Q] / I(G, \phi)$ is Cohen-Macaulay.

We show now that every 2-dimensional Cohen-Macaulay monomial ideal $I$ with minimal multiplicity is of the form $I(G, \phi)$. Let $Q$ be the set of variables whose square is in $I$ and $V$ the remaining variables. We argue by induction on the cardinality of $Q$. If $Q$ is empty, then $I$ is square-free and we know that $I$ is associated to a tree. If $Q$ is not empty, let $q \in Q$. Write $I+(q)=J+(q)$ with $J$ a monomial ideal not involving the variable $q$. By Lemma 4.3 we know that $J \subset K[V, Q \backslash\{q\}]$ is 2-dimensional Cohen-Macaulay with minimal multiplicity. Therefore there exists a tree $G$ with vertices $V$ and edges $E$, and a map

$$
\phi^{\prime}: Q \backslash\{q\} \rightarrow E
$$

so that $J=I\left(G, \phi^{\prime}\right)$. On the other hand $I=J+q(I: q)$ and by Corollary 4.4 there are $x, y \in V$ so that $I:(q)=(Q \cup V \backslash\{x, y\})$ with $x y \notin I$. Hence $\{x, y\}$ belongs to $E$ and we extend $\phi^{\prime}$ to $Q$ by sending $q$ to $\{x, y\}$. Call the resulting map $\phi$. By construction, $I(G, \phi)=I$.

Now we come to the second step of our classification: to describe the CohenMacaulay monomial initial ideals of $P$. Let $I$ be a monomial Cohen-Macaulay initial ideal of $P$. Since $P$ is 2-dimensional with a short $h$-vector, so is $I$. By what we have shown above, there exist $G$ and $\phi$ so that $I=I(G, \phi)$. We want to describe which pairs $(G, \phi)$ arise in this way.

Lemma 4.8. Let I be a monomial initial ideal of $P$ generated in degree 2.

(1) For every $v, 0 \leq v \leq 2 d$, there exists exactly one monomial $t_{i} t_{j}$ such that $i+j=v$ and $t_{i} t_{j} \notin I$. In particular, $t_{0}^{2}$ and $t_{d}^{2}$ are not in $I$.

(2) Let $0 \leq i<j<k \leq d$. Assume that $t_{i}^{2}, t_{j}^{2}$ and $t_{k}^{2}$ are not in $I$. Then $t_{i} t_{k} \in I$.

(3) Let $0 \leq i<j<k \leq d$. Assume that $t_{i}^{2} \notin I$ and $t_{s} t_{j} \notin I$ for every $s$ with $i \leq s \leq j$. Then $t_{i} t_{k} \in I$.

Proof. (1) By definition, $K\left[t_{0}, \ldots, t_{d}\right] / P$ is bigraded by setting $\operatorname{deg} t_{i}=(d-$ $i, i)$ and 1-dimensional in each bihomogeneous component. Therefore the ring $K\left[t_{0}, \ldots, t_{d}\right] / I$ is also bigraded and 1-dimensional in each bihomogeneous component. This proves the assertion. (2) Since $t_{i}^{k-j} t_{k}^{j-i}-t_{j}^{k-i} \in P$, we have that either $t_{i}^{k-j} t_{k}^{j-i}$ or $t_{j}^{k-i}$ belongs to $I$. If $t_{j}^{k-i}$ belongs to $I$, since $I$ is generated in 
degree 2, then $t_{j}^{2}$ belongs to $I$; a contradiction. So we have $t_{i}^{k-j} t_{k}^{j-i} \in I$. Since $t_{i}^{2}$ and $t_{k}^{2}$ are not in $I$ we conclude that $t_{i} t_{k} \in I$. (3) We show that there exist a positive integer $a$ and $i \leq s \leq j$ so that $t_{i}^{a} t_{k}-t_{s} t_{j}^{a} \in P$. Then since, by assumption, the factors of degree 2 of $t_{s} t_{j}^{a}$ are not in $I$, we have that a factor of degree 2 of $t_{i}^{a} t_{k}$ is in $I$. Since $t_{i}^{2} \notin I$, it follows that $t_{i} t_{k} \in I$. To show that $a$ and $s$ as above exist, note that the condition $t_{i}^{a} t_{k}-t_{s} t_{j}^{a} \in P$ translates into $s=k-a(j-i)$. So we have to show that there exists a positive integer $a$ such that

$$
i \leq k-a(j-i) \leq j,
$$

or equivalently

$$
\frac{k-j}{j-i} \leq a \leq \frac{k-i}{j-i} .
$$

But this interval has length 1 so it contains an integer which is positive since $i<$ $j<k$.

The matrix $T_{d}$ appearing in the following theorem is defined in Section 2.

Theorem 4.9. Let I be a monomial ideal of $K\left[t_{0}, \ldots, t_{d}\right]$. The following conditions are equivalent.

(1) There exists a sequence $0=i_{0}<i_{1}<i_{2} \cdots<i_{k}=d$ such that $I(G, \phi)=I$ where $V=\left\{t_{i_{0}}, t_{i_{1}}, \ldots, t_{i_{k}}\right\}, Q=\left\{t_{0}, \ldots, t_{d}\right\} \backslash V, G$ is the tree (a line) with vertex set $V$ and with edge set $E=\left\{\left\{t_{i_{j}}, t_{i_{j+1}}\right\}: 0 \leq j \leq k-1\right\}$ and the map $\phi: Q \rightarrow E$ sends $t_{s}$ to $\left\{t_{i_{j}}, t_{i_{j+1}}\right\}$ where $i_{j}<s<i_{j+1}$.

(2) There exists a sequence $0=i_{0}<i_{1}<i_{2} \cdots<i_{k}=d$ such that I is generated by

(a) the main diagonals $t_{v-1} t_{r}$ of the 2-minors of the matrix $T_{d}$ with column indices $v, r$ such that $v \leq i_{j}<r$ for some $j$, and

(b) the antidiagonals $t_{v} t_{r-1}$ of the 2-minors of the matrix $T_{d}$ with column indices $v, r$ such that $i_{j}<v<r \leq i_{j+1}$ for some $j$.

(3) I is a Cohen-Macaulay initial ideal of $P$.

Proof. To prove that (1) and (2) are equivalent is just a direct check. To prove that (1) and (2) imply (3), it is enough to describe a term order $\tau$ such that

$$
\operatorname{in}_{\tau}(P)=I(G, \phi) .
$$

Indeed, the inclusion $\operatorname{in}_{\tau}(P) \supseteq I(G, \phi)$ is enough because the two ideals have the same Hilbert function. To do so, consider a vector $b=\left(b_{1}, b_{2}, \ldots, b_{d}\right)$ such that $b_{r}>b_{v}$ if $v \leq i_{j}<r$ for some $j$ and $b_{v}>b_{r}$ if $i_{j}<v<r \leq i_{j+1}$ for some $j$. In Remark 4.10 we will show a canonical way to construct such a vector. Define $a=\left(a_{0}, a_{1}, \ldots, a_{d}\right)$ by setting $a_{0}=0$ and $a_{i}=\sum_{j=1}^{i} b_{j}$. Consider a term order $\tau$ refining (no matter how) the order defined by the vector $a$. We claim that the initial 
term with respect to $\tau$ of the minor with column indices $v, r(v<r)$ of the matrix $T_{d}$ is the one prescribed by (2). The minor with those column indices is $t_{v-1} t_{r}-t_{v} t_{r-1}$. The weights with respect to $a$ of the two terms are $a_{v-1}+a_{r}$ and $a_{v}+a_{r-1}$. Hence $a_{v-1}+a_{r}>a_{v}+a_{r-1}$ if and only if $b_{r}>b_{v}$ and $a_{v-1}+a_{r}<a_{v}+a_{r-1}$ if and only if $b_{r}<b_{v}$. Therefore, by construction, the initial forms of the minors of $T_{d}$ with respect to $a$ are exactly the monomials prescribed by (2).

We prove now that (3) implies (1). Assume that $I$ is a Cohen-Macaulay initial ideal of $P$. Then $I$ has regularity 2 and, in particular, is generated by elements of degree 2. Set $V=\left\{t_{i}: t_{i}^{2} \notin I\right\}$ and $Q=\left\{t_{i}: t_{i}^{2} \in I\right\}$. We know by Lemma 4.8 (1) that $t_{0}$ and $t_{d}$ are in $V$. So with $0=i_{0}<i_{1}<i_{2} \cdots<i_{k}=d$ we may write $V=\left\{t_{i_{0}}, t_{i_{1}}, \ldots, t_{i_{k}}\right\}$. Since $I$ is 2 -dimensional Cohen-Macaulay with minimal multiplicity, it has the form $I=I\left(G^{\prime}, \phi^{\prime}\right)$. We prove that $G^{\prime}=G$ and $\phi^{\prime}=\phi$ where $G$ and $\phi$ are those described in (1). Note that, by Lemma 4.8 (2), we have that $t_{i_{v}} t_{i_{r}} \in I$ whenever $r-v>1$ so that $\left\{t_{v}, t_{r}\right\}$ is not an edge of $G^{\prime}$. This implies that the underlying tree $G^{\prime}$ is exactly the line $G$. It remains to prove that $\phi=\phi^{\prime}$. In other words, we have to prove that for every $t_{j} \in Q$, say $i_{r}<j<i_{r+1}$, one has $t_{j} t_{i_{r}} \notin I$ and $t_{j} t_{i_{r+1}} \notin I$. By contradiction, let $j$ be the smallest element of $Q$ that does not satisfy the required condition, say $i_{r}<j<i_{r+1}$.

Claim. If $0 \leq v<r$ and $q \geq j$ then $t_{i_{v}} t_{q} \in I$.

To prove the claim, note that, by the choice of $j$, we know that $t_{s} t_{i_{v+1}} \notin I$ for every $i_{v} \leq s \leq i_{v+1}$ and $i_{v+1} \leq i_{r}<j$. Therefore we may apply Lemma 4.8 (3) to the indices $i_{v}, i_{v+1}, q$ and we conclude that $t_{i_{v}} t_{q} \in I$. In particular, for $q=j$ the claim says that $t_{i_{v}} t_{j} \in I$ for every $v<r$. So $\phi^{\prime}\left(t_{j}\right)$ must be an edge of the form $\left\{t_{i_{u}}, t_{i_{u+1}}\right\}$ with $u>r$. It follows that $t_{i_{r}} t_{j} \in I$. But, according to Lemma 4.8 (1), there exists (exactly one but we do not need this) a monomial $t_{a} t_{b}$ (say $a \leq b$ ) such that $a+b=i_{r}+j$ and $t_{a} t_{b} \notin I$. We distinguish now three cases.

Case 1. $i_{r}<a$ and $b<j$, so that $a$ and $b$ are both in $Q$. A contradiction (the square of $Q$ is contained in $I$ ).

Case 2. $a<i_{r}, b>j$ and $a \in V$. The claim above says that $t_{a} t_{b} \in I$. A contradiction.

Case 3. $a<i_{r}, b>j$ and $a \in Q$. Say $i_{u}<a<i_{u+1}$. By induction we know that $\left\{t_{v}: t_{v} t_{a} \notin I\right\}=\left\{t_{i_{u}}, t_{i_{u+1}}\right\}$ and therefore $b>j>i_{r} \geq i_{u+1}$. So $t_{a} t_{b} \in I$. A contradiction.

This concludes the proof.

Remark 4.10. Given $0=i_{0}<i_{1}<i_{2} \cdots<i_{k}=d$, in the proof of Theorem 4.9 we have used a vector $b=\left(b_{1}, b_{2}, \ldots, b_{d}\right) \in \mathbb{Q}_{\geq 0}^{d}$ with the property that $b_{r}>b_{v}$ if $v \leq i_{j}<r$ for some $j$ and $b_{v}>b_{r}$ if $i_{j}<v<r \leq i_{j+1}$ for some $j$. Of course there are many vectors with this property. But there is just one "permutation" vector, whose entries are the numbers $1, \ldots, d$ permuted in some way, with this property. It arises 
as follows: for $j=1, \ldots, k$, consider the vector $c_{j}=\left(i_{j}, i_{j}-1, \ldots, i_{j-1}+1\right)$ and define the vector $b$ to be the concatenation of $c_{1}, c_{2} \ldots, c_{k}$.

The following theorem summarizes what we have proved so far.

Theorem 4.11. (1) The ideal $P$ has exactly $2^{d-1}$ distinct Cohen-Macaulay monomial initial ideals.

(2) They are in bijective correspondence with the sequences $0=i_{0}<i_{1}<\cdots<$ $i_{k}=d$, namely with their radical.

(3) Each of them can be obtained with a term order associated to a vector $a=$ $\left(a_{0}, a_{1}, \ldots, a_{d}\right) \in \mathbb{N}^{d+1}$ with $0=a_{0}<a_{1}<\cdots<a_{d}$.

(4) Each of them is obtained by taking the appropriate initial terms of the 2minors of the matrix $T_{d}$.

(5) The reduced Gröbner basis of $P$ giving the Cohen-Macaulay initial ideal corresponding to the sequence $0=i_{0}<i_{1}<i_{2} \cdots<i_{k}=d$ is the set of polynomials

$$
\begin{aligned}
\underline{t_{s} t_{r}}-t_{i_{v}} t_{s+r-i_{v}}, & \text { if } \quad 2 i_{v} \leq s+r \leq i_{v}+i_{v+1}, \\
\underline{t_{s} t_{r}}-t_{i_{v+1}} t_{s+r-i_{v+1}}, & \text { if } \quad i_{v}+i_{v+1} \leq s+r \leq 2 i_{v+1},
\end{aligned}
$$

where the initial terms are underlined.

Definition 4.12. For every sequence $\mathbf{i}=\left(i_{0}, i_{1}, \ldots, i_{k}\right)$ with $0=i_{0}<i_{1}<\cdots<i_{k}=d$ we denote by $C(\mathbf{i})$ the open cone in $\mathbb{Q}_{\geq 0}^{d+1}$ of the points $\left(a_{0}, \ldots, a_{d}\right)$ satisfying the inequalities

$$
\begin{array}{rll}
a_{s}+a_{r}>a_{i_{v}}+a_{s+r-i_{v}}, & \text { if } & 2 i_{v} \leq s+r \leq i_{v}+i_{v+1}, \\
a_{s}+a_{r}>a_{i_{v+1}}+a_{s+r-i_{v+1}}, & \text { if } & i_{v}+i_{v+1} \leq s+r \leq 2 i_{v+1},
\end{array}
$$

and by $\overline{C(\mathbf{i})}$ the corresponding closed cone, that is, the subset of $\mathbb{Q}_{\geq 0}^{d+1}$ described by the inequalities above where $>$ is replaced throughout by $\geq$.

We are ready to state and prove the main theorem of this section.

Theorem 4.13. Let $a \in \mathbb{Q}_{\geq 0}^{d+1}$. Then $\operatorname{in}_{a}(P)$ is Cohen-Macaulay if and only if $\operatorname{in}_{a}(P)$ has a Cohen-Macaulay initial monomial ideal. In other words,

$$
\left\{a \in \mathbb{Q}_{\geq 0}^{d+1}: \operatorname{in}_{a}(P) \text { is Cohen-Macaulay }\right\}=\bigcup_{\mathbf{i}} \overline{C(\mathbf{i})}
$$

where the union is indexed by the $2^{d-1}$ sequences $\mathbf{i}=\left(0=i_{0}<i_{1}<\ldots<i_{k}=d\right)$.

Proof. First we prove the inclusion $\supseteq$. Let $a \in \overline{C(\mathbf{i})}$. To see that $\operatorname{in}_{a}(P)$ is CohenMacaulay it is enough to prove that it has a Cohen-Macaulay initial ideal. Just take $a^{\prime}$ in the open cone $C(\mathbf{i})$ and check that $\operatorname{in}_{a^{\prime}}\left(\operatorname{in}_{a}(P)\right)=\operatorname{in}_{a^{\prime}}(P)$. This is easy since it is enough to check that $\operatorname{in}_{a^{\prime}}\left(\operatorname{in}_{a}(P)\right) \supseteq \operatorname{in}_{a^{\prime}}(P)$. 
In order to prove the opposite inclusion, let $a \in \mathbb{Q}_{\geq 0}^{d+1}$ be such that $\operatorname{in}_{a}(P)$ is Cohen-Macaulay. We have to show that $a \in \overline{C(\mathbf{i})}$ for some i. We know already that if $\operatorname{in}_{a}(P)$ is monomial then $a \in C(\mathbf{i})$ where $\mathbf{i}$ is the sequence of the indices $i$ such that no power of $t_{i}$ belongs to $\operatorname{in}_{a}(P)$. So we are left with the case $\operatorname{in}_{a}(P)$ is nonmonomial. To treat this case we first note that, without loss of generality, we may assume that $a=\left(a_{0}, \ldots, a_{d}\right)$ with $a_{i} \in \mathbb{N}, a_{0}=0$, and $a_{i}<a_{i+1}$. This is because cleaning denominators and adding to $a$ multiples of the vectors $(1, \ldots, 1)$ and $(0,1,2, \ldots, d)$ change neither $\operatorname{in}_{a}(P)$ nor the membership in the cones. Then we may associate to $a$ a lex-segment ideal $L$ in $R=K[x, y]$ as described in Section 3. We compute the deviation $V(L)$ of $L$ in terms of the $a_{i}$ 's. It is well-know, see for instance [Delfino et al. 2003], that the multiplicity $e_{0}(L)$ of $L$ is twice the area of the region $\mathbb{R}_{+}^{2} \backslash \operatorname{New}(L)$ where $\operatorname{New}(L)$ is the Newton polytope of $L$, that is the convex hull of the set of elements $(a, b) \in \mathbb{N}^{2}$ such that $x^{a} y^{b} \in L$. To determine $e_{0}(L)$ we describe the vertices of $\operatorname{New}(L)$. The generators of $L$ are the elements $x^{d-i} y^{a_{i}}$. Set $i_{0}=0$ and assume that $i_{t}<d$ is already defined. Then we set

$$
\begin{gathered}
m=\min \left\{\left(a_{j}-a_{i_{t}}\right) /\left(j-i_{t}\right): j=i_{t}+1, \ldots, d\right\}, \\
i_{t+1}=\max \left\{j: i_{t}+1<j \leq d \text { and }\left(a_{j}-a_{i_{t}}\right) /\left(j-i_{t}\right)=m\right\} .
\end{gathered}
$$

The procedure stops when we have reached, say after $k$ steps, $i_{k}=d$. By construction the points $\left(d-c, a_{c}\right)$ with $c \in\left\{i_{0}, i_{1}, \ldots, i_{k}\right\}$ are the vertices of $\operatorname{New}(L)$. Taking into account that twice the area of the triangle with vertices $(0,0)$, $\left(d-i_{t}, a_{i_{t}}\right),\left(d-i_{t+1}, a_{i_{t+1}}\right)$ is $a_{i_{t+1}}\left(d-i_{t}\right)-a_{i_{t}}\left(d-i_{t+1}\right)$ and that $a_{0}=0, i_{0}=0$ we obtain

$$
e_{0}(L)=a_{0}\left(i_{1}-i_{0}\right)+\sum_{t=1}^{k-1} a_{i_{t}}\left(i_{t+1}-i_{t-1}\right)+a_{i_{k}}\left(i_{k}-i_{k-1}\right) .
$$

For $j=0, \ldots, 2 d$ set $\alpha_{j}=\min \left\{a_{s}+a_{r}: s+r=j\right\}$ and

$$
\beta_{j}= \begin{cases}a_{i_{t}}+a_{j-i_{t}} & \text { if } \quad 2 i_{t} \leq j \leq i_{t}+i_{t+1} \\ a_{i_{t+1}}+a_{j-i_{t+1}} & \text { if } \quad i_{t}+i_{t+1} \leq j \leq 2 i_{t+1} .\end{cases}
$$

Since $\operatorname{dim}_{K}(R / L)=\sum_{i=0}^{d} a_{i}$ and $\operatorname{dim}_{K}\left(R / L^{2}\right)=\sum_{i=0}^{2 d} \alpha_{i}$ we have

$$
\begin{aligned}
V(L) & =e_{0}(L)-\sum_{i=0}^{2 d} \alpha_{i}+2 \sum_{i=0}^{d} a_{i} \\
& =e_{0}(L)-\sum_{i=0}^{2 d} \beta_{i}+2 \sum_{i=0}^{d} a_{i}+\sum_{i=0}^{2 d}\left(\beta_{i}-\alpha_{i}\right) \\
& =Z+\sum_{i=0}^{2 d}\left(\beta_{i}-\alpha_{i}\right)
\end{aligned}
$$


where

$$
Z=a_{0}\left(i_{1}-i_{0}\right)+\sum_{t=1}^{k-1} a_{i_{t}}\left(i_{t+1}-i_{t-1}\right)+a_{i_{k}}\left(i_{k}-i_{k-1}\right)-\sum_{i=0}^{2 d} \beta_{i}+2 \sum_{i=0}^{d} a_{i} .
$$

We claim that $Z=0$ identically as a linear form in the $a_{i}$ 's. This can be checked directly. That $Z=0$ follows also from the fact that $Z$, as a linear function in the $a_{i}$ 's, computes the deviation $V(H)$ where $H$ is any lex-segment with associated vector $a$ in the cone $C(\mathbf{i})$. Since every such lex-segment ideal has a Cohen-Macaulay associated graded ring, we have $V(H)=0$. Therefore $Z$ vanishes when evaluated at the points of $C(\mathbf{i}) \cap\left\{a \in \mathbb{N}^{d+1}: 0=a_{0}<a_{1}<\cdots<a_{d}\right\}$ and so it vanishes identically. Summing up, we have

$$
V(L)=\sum_{i=0}^{2 d}\left(\beta_{i}-\alpha_{i}\right)
$$

Now, by assumption $\operatorname{in}_{a}(P)$ is Cohen-Macaulay, thus by Proposition $3.9 \operatorname{gr}_{L}(R)$ is Cohen-Macaulay and by Theorem 3.2 $V(L)=0$. Since $\beta_{i} \geq \alpha_{i}$ for every $i$, it follows that $\beta_{i}=\alpha_{i}$ for every $i$, which in turn implies that $a \in \overline{C(\mathbf{i})}$.

Remark 4.14. Let $a=\left(a_{0}, \ldots, a_{d}\right)$ be the vector associated to a lex-segment ideal $L$. Denote by $Y$ the convex hull of $\left\{(i, j): x^{i} y^{j} \in L\right\}$, by $V$ the set of the vertices of $Y$ and by $V^{\prime}$ the set of the elements $\left(d-i, a_{i}\right)$ belonging to the lower boundary of $Y$. Clearly $V \subseteq V^{\prime}$. Assume that $\operatorname{gr}_{L}(R)$ is Cohen-Macaulay. The proof above shows that $a \in \overline{C(\mathbf{i})}$ where $\left\{\left(d-j, a_{j}\right): j \in \mathbf{i}\right\}=V$. The same argument shows also that $a \in \overline{C(\mathbf{i})}$ for every $\mathbf{i}$ such that $V \subseteq\left\{\left(d-j, a_{j}\right): j \in \mathbf{i}\right\} \subseteq V^{\prime}$. In particular, $a$ belongs to $2^{u}$ of the cones $\overline{C(\mathbf{i})}$ where $u=\# V^{\prime}-\# V$.

The next example illustrates the remark above.

Example 4.15. Let $a=\left(0,2,4, a_{3}, a_{4}, a_{5}\right)$ with

$$
4<a_{3}<a_{4}<a_{5}, \quad a_{3}>4+\left(a_{5}-4\right) / 3, \quad a_{4}>4+2\left(a_{5}-4\right) / 3 .
$$

Then $V=\left\{(5,0),(3,4),\left(0, a_{5}\right)\right\}$ and $V^{\prime}=V \cup\{(4,2)\}$. By the remark above we have that if $\operatorname{in}_{a}(P)$ is Cohen-Macaulay then $a \in \overline{C(\mathbf{i})} \cap \overline{C(\mathbf{j})}$ with $\mathbf{i}=(0,1,2,5)$ and $\mathbf{j}=(0,2,5)$. In this case the Cohen-Macaulay property is equivalent to the inequalities

$$
a_{4} \geq a_{3}+2, \quad a_{5} \geq a_{4}+2, \quad 2 a_{3} \geq a_{4}+4, \quad 2 a_{4} \geq a_{3}+a_{5} .
$$

Definition 4.16. Let $\sigma \in S_{d}$ be a permutation. We may associate to $\sigma$ a cone

$$
C_{\sigma}=\left\{a \in \mathbb{Q}_{\geq 0}^{d+1}: b_{\sigma^{-1}(1)}<\cdots<b_{\sigma^{-1}(d)}\right\}
$$

where $b_{i}=a_{i}-a_{i-1}$. We call $C_{\sigma}$ the permutation cone associated to $\sigma$. 
Remark 4.17. As shown in the proof of Theorem 4.9 and Remark 4.10 each cone $C$ (i) contains a specific permutation cone $C_{\sigma}$. The permutations involved in the construction are indeed permutations avoiding the patterns " 231 " and " 312 ". More precisely, there is a bijective correspondence between the permutations $\sigma \in S_{d}$ avoiding the patterns " 231 " and " 312 " and the cones $C(\mathbf{i})$ so that $C(\mathbf{i}) \supseteq C_{\sigma}$. However, as we will see, the inclusion $C_{\sigma} \subseteq C(\mathbf{i})$ can be strict in general. The study of permutation patterns is an important subject in combinatorics; see for instance [Wilf 2002].

The following example illustrates Theorem 4.11.

Example 4.18. Suppose $d=6$ and take the sequence $\mathbf{i}=\left(i_{0}=0, i_{1}=3, i_{2}=4, i_{3}=6\right)$. The corresponding Cohen-Macaulay initial ideal $I$ of $P$ is obtained by dividing the matrix $T_{6}$ into blocks (from column $i_{v}+1$ to $i_{v+1}$ )

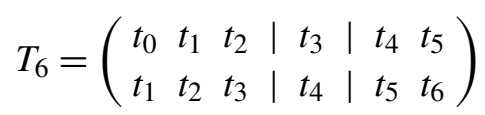

and then taking the antidiagonals of minors whose columns belong to the same block:

$$
t_{1}^{2}, t_{1} t_{2}, t_{2}^{2}, t_{5}^{2}
$$

and the main diagonals from minors whose columns belong to different blocks:

$$
t_{0} t_{4}, t_{0} t_{5}, t_{0} t_{6}, t_{1} t_{4}, t_{1} t_{5}, t_{1} t_{6}, t_{2} t_{4}, t_{2} t_{5}, t_{2} t_{6}, t_{3} t_{5}, t_{3} t_{6} \text {. }
$$

The ideal $I$ is the initial ideal of $P$ with respect to every term order refining the weight $a=(0,3,5,6,10,16,21)$ obtained from the "permutation" vector

$$
\sigma=(3,2,1|4| 6,5) \in S_{6}
$$

by setting $a_{0}=0$ and $a_{i}=\sum_{j=1}^{i} \sigma_{j}$. With respect to this term order the 2-minors of $T_{6}$ are a Gröbner basis of $P$ but not the reduced Gröbner basis. The corresponding reduced Gröbner basis is

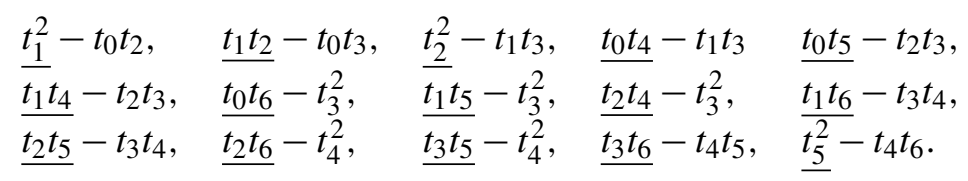

So for every vector $a=\left(a_{0}, a_{1}, \ldots, a_{6}\right) \in \mathbb{Q}_{\geq 0}^{7}$ satisfying the system of linear inequalities

$$
\begin{array}{llll}
2 a_{1}>a_{0}+a_{2} *, & a_{1}+a_{2}>a_{0}+a_{3}, & 2 a_{2}>a_{1}+a_{3} *, & a_{0}+a_{4}>a_{1}+a_{3} *, \\
a_{0}+a_{5}>a_{2}+a_{3}, & a_{1}+a_{4}>a_{2}+a_{3}, & a_{0}+a_{6}>2 a_{3}, & a_{1}+a_{5}>2 a_{3}, \\
a_{2}+a_{4}>2 a_{3}, & a_{1}+a_{6}>a_{3}+a_{4}, & a_{2}+a_{5}>a_{3}+a_{4}, & a_{2}+a_{6}>2 a_{4}, \\
a_{3}+a_{5}>2 a_{4}, & a_{3}+a_{6}>a_{4}+a_{5} *, & 2 a_{5}>a_{4}+a_{6} *, &
\end{array}
$$


we have $\operatorname{in}_{a}(P)=I$. The 15 linear homogeneous inequalities above define the open Cohen-Macaulay cone $C(\mathbf{i})$. The description is however far from being minimal. The inequalities marked with $*$ are indeed sufficient to describe $C(\mathbf{i})$. In terms of $b_{i}=a_{i}-a_{i-1}$ the inequalities can be described by $b_{3}<b_{2}<b_{1}<b_{4}<b_{6}<b_{5}$, that is, $C(\mathbf{i})=C_{\sigma}$.

Remark 4.19. (1) There exist Cohen-Macaulay ideals of dimension 2 with minimal multiplicity and without Cohen-Macaulay initial monomial ideals in the given coordinates. For instance, let $J$ be the ideal of $K\left[t_{0}, \ldots, t_{4}\right]$ generated by the 2-minors of the matrix

$$
\left(\begin{array}{cccc}
t_{0} & t_{2} & t_{4}-t_{0} & 0 \\
t_{1} & t_{3} & 0 & t_{4}+t_{0}
\end{array}\right)
$$

Then $J$ has the expected codimension and hence it is 2-dimensional CohenMacaulay with minimal multiplicity. No monomial initial ideal of $J$ is quadratic since the degree 2 part of every monomial initial ideal has codimension 2. Hence no monomial initial ideal of $J$ is Cohen-Macaulay. This example shows that Theorem 4.13 does not hold for 2-dimensional binomial CohenMacaulay ideals with minimal multiplicity.

(2) in $_{a}(P)$ can be monomial, quadratic and non-Cohen-Macaulay. For example, for $d=4$ the ideal $I=\left(t_{3}^{2}, t_{2} t_{3}, t_{1} t_{3}, t_{0} t_{4}, t_{0} t_{3}, t_{1}^{2}\right)$ is a non-Cohen-Macaulay (indeed nonpure) monomial initial ideal of $P$. The corresponding cone is described in terms of $b_{i}=a_{i}-a_{i-1}$ by the inequalities $b_{3}>b_{1}>b_{2}>b_{4}$ and $b_{3}+b_{4}>b_{1}+b_{2}$.

(3) $\operatorname{in}_{a}(P)$ can be quadratic with linear 1-syzygies and not Cohen-Macaulay. For instance, with $d=7$ the ideal generated by

$$
\begin{aligned}
& t_{2}^{2}, t_{4}^{2}, t_{6}^{2}, t_{1} t_{2}, t_{0} t_{4}, t_{0} t_{5}, t_{1} t_{4}, t_{0} t_{6}, t_{1} t_{5}, t_{2} t_{4}, t_{0} t_{7}, t_{1} t_{6}, t_{3} t_{4}, \\
& t_{1} t_{7}, t_{2} t_{6}, t_{3} t_{6}, t_{3} t_{7}, t_{4} t_{6}, t_{1}^{2}+t_{0} t_{2},-t_{4} t_{5}+t_{2} t_{7},-t_{5} t_{6}+t_{4} t_{7}
\end{aligned}
$$

is an initial ideal of $P$ with linear 1-syzygies and a nonlinear 2-syzygy.

(4) We do not know any example as the one in (3) if we further assume that $\operatorname{in}_{a}(P)$ is a monomial ideal. Note however that 2-dimensional non-Cohen-Macaulay quadratic monomial ideals with a short $h$-vector and linear 1-syzygies exist, for example $\left(t_{1} t_{3}, t_{1} t_{5}, t_{0} t_{2}, t_{2} t_{5}, t_{0} t_{3}, t_{2}^{2}, t_{2} t_{4}, t_{2} t_{3}, t_{0} t_{4}, t_{4} t_{5}\right)$.

Remark 4.20. As Rekha Thomas pointed out to us, one can deduce from results in [Hoşten and Thomas 2003; O'Shea and Thomas 2005] that $P$ has exactly one Cohen-Macaulay monomial initial ideal for each regular triangulation of the underlying point configuration $A$. In [Hoşten and Thomas 2003, Theorem 5.5(ii)] it is proved that for every regular triangulation of $A$ there exists exactly one initial ideal 
having no embedded primes (they are called Gomory initial ideals in that paper). In [O'Shea and Thomas 2005] it is proved that every Gomory initial ideal coming from a $\Delta$-normal configuration is Cohen-Macaulay. Since every triangulation of $A$ is $\Delta$-normal, one can conclude that the Gomory ideals of $P$ are indeed CohenMacaulay. Hence these results imply that $P$ has exactly $2^{d-1}$ Cohen-Macaulay monomial initial ideals.

\section{Contracted ideals whose associated graded ring is Cohen-Macaulay}

In this section we use the results of Section 4 to solve the problem (2) mentioned in Section 1. Since the ideal $P$ is homogeneous with respect to the vectors $(1,1, \ldots, 1)$ and $(0,1,2, \ldots, d)$ of $\mathbb{Q}^{d+1}$, each cone of the Gröbner fan of $P$ is determined by its intersection with

$$
W_{d}=\left\{\left(a_{0}, a_{1}, \ldots, a_{d}\right) \in \mathbb{N}^{d+1}: 0=a_{0}<a_{1}<\cdots<a_{d}\right\} .
$$

As explained in Section 3, $W_{d}$ parametrizes the lex-segment ideals of initial degree $d$. For a given $d \in \mathbb{N}, d>0$, we set

$$
C M_{d}=\left\{a \in \mathbb{Q}_{\geq 0}^{d+1}: \operatorname{in}_{a}(P) \text { is Cohen-Macaulay }\right\}
$$

the "Cohen-Macaulay region" of the Gröbner fan of $P$. According to Theorem 4.13 we have

$$
C M_{d}=\bigcup_{\mathbf{i}} \overline{C(\mathbf{i})}
$$

where the union is extended to all of the $2^{d-1}$ sequences $\mathbf{i}=\left(0=i_{0}<i_{1}<\ldots<i_{k}=d\right)$.

Theorem 5.1. Let $d_{1}, \ldots, d_{s}$ be positive integers and $a_{1}, \ldots, a_{s}$ be vectors such that $a_{i} \in W_{d_{i}}$. Let $\ell_{1}, \ldots, \ell_{s}, z$ be linear forms in $R=K[x, y]$ such that each pair of them is linearly independent. For every $i=1, \ldots, s$, consider the lex-segment ideals $L_{i}$ associated to $a_{i}$ with respect to $\ell_{i}, z$, that is,

$$
L_{i}=\left(\ell_{i}^{d_{i}-j} z^{a_{i j}}: j=0, \ldots, d_{i}\right) .
$$

Set $I=L_{1} \cdots L_{s}$. We have

(1) I is contracted and every homogeneous contracted ideal in $R=K[x, y]$ arises in this way, and

(2) $\operatorname{gr}_{I}(R)$ is Cohen-Macaulay if and only if $a_{i} \in C M_{d_{i}}$ for all $i=1, \ldots, s$.

Proof. (1) is a restatement of Zariski's factorization theorem for contracted ideals. (2) follows from Theorem 3.3, Proposition 3.9 and Theorem 4.13.

Theorem 5.1 can be generalized as follows. 
Theorem 5.2. Let $I \subset K[x, y]$ be a monomial ideal (not necessarily contracted) and let $a=\left(a_{0}, \ldots, a_{d}\right)$ be its associated sequence. Then $\operatorname{gr}_{I}(R)$ is CohenMacaulay if and only if $a \in C M_{d}$.

Proof. If $a$ is strictly increasing, then $I$ is a lex-segment ideal. Hence $I$ is contracted and the statement is a special case of Theorem 5.1. If $a$ is not strictly increasing, then we set $a^{\prime}=a+(0,1, \ldots, d)$ and let $L$ be the monomial ideal associated to $a^{\prime}$. Since $a^{\prime}$ is strictly increasing, $L$ is a lex-segment ideal. The cones $C(\mathbf{i})$ are described by the inequalities that are homogeneous with respect to $(0,1, \ldots, d)$. Therefore $a$ belongs to $C M_{d}$ if and only if $a^{\prime}$ does. By construction, $I$ is the quadratic transform of the contracted ideal $L$ in the sense of [Conca et al. 2005, Sect. 3]. Further we know that depth $\operatorname{gr}_{I}(R)=\operatorname{depth} \operatorname{gr}_{L}(R)$ according to [Conca et al. 2005, Thm. 3.12]. In summary, $\operatorname{gr}_{I}(R)$ is Cohen-Macaulay if and only if $\operatorname{gr}_{L}(R)$ is Cohen-Macaulay if and only if $a^{\prime} \in C M_{d}$ if and only if $a \in C M_{d}$.

Remark 5.3. (1) In $K[x, y]$ denote by $C$ the class of contracted ideals, by $C_{1}$ the class of the ideals in $C$ with Cohen-Macaulay associated graded ring and by $C_{2}$ the class of integrally closed ideals. We have $C \supset C_{1} \supset C_{2}$. One knows that $C$ and $C_{2}$ are closed under product. On the other hand $C_{1}$ is not: the lex-segment ideals associated to the sequences $(0,4,6,7)$ and $(0,2)$ belong to $C_{1}$ and their product does not. However, $C_{1}$ is closed under powers: if $I \in C_{1}$ then $I^{k} \in C_{1}$. This can be seen, for instance, by looking at the Hilbert function of $I$. Furthermore we will show in Section 7 that a certain subset of $C_{1}$ is closed under product.

(2) For a lex-segment ideal $L$ in $K[x, y]$ we have seen that $\operatorname{gr}_{L}(R)$ and $F(L)$ have the same depth. We believe that $\operatorname{gr}_{I}(R)$ and $F(I)$ have the same depth for every contracted ideal $I$. In [D'Cruz et al. 1999, Thm. 3.7, Cor. 3.8] D'Cruz, Raghavan and Verma proved that the Cohen-Macaulayness of $\operatorname{gr}_{I}(R)$ is equivalent to that of $F(I)$. Note however that for a monomial ideal $I$ the rings $\operatorname{gr}_{I}(R)$ and $F(I)$ might have different depth. For instance, for the ideal $I$ associated to $(0,2,2,3)$ one has depth $\operatorname{gr}_{I}(R)=1$ and depth $F(I)=2$.

Remark 5.4. Two of the cones of the Cohen-Macaulay region $C M_{d}$ are special as they correspond to opposite extreme selections.

(1) If $\mathbf{i}=(0,1,2, \ldots, d)$, then the closed cone $\overline{C(\mathbf{i})}$ is described by the inequality system $a_{i}+a_{j} \geq a_{u}+a_{v}$ with $u=\lfloor(i+j) / 2\rfloor, v=\lceil(i+j) / 2\rceil$ for every $i, j$ or, equivalently, by $b_{i+1} \geq b_{i}$ for every $i=1, \ldots, d-1$. In other words, $C(\mathbf{i})$ equals its permutation cone $C_{i d}$, where $i d \in S_{d}$ is the identity permutation. In this case the initial ideal of $P$ is $\left(t_{i} t_{j}: j-i>1\right)$ and it can be realized by the lex-order $t_{0}<t_{1}<\cdots<t_{d}$ or by the lex-order $t_{0}>t_{1}>\cdots>t_{d}$. This is the only radical monomial initial ideal of $P$. The points in $W_{d} \cap \overline{C(\mathbf{i})}$ correspond 
to integrally closed lex-segment ideals. Indeed, they are the products of $d$ complete intersections of type $\left(x, y^{u}\right)$.

(2) If $\mathbf{i}=(0, d)$ then the closed cone $\overline{C(\mathbf{i})}$ is described by the inequality system

$$
\begin{aligned}
a_{i}+a_{j} \geq a_{0}+a_{i+j}, & \text { if } i+j \leq d \quad \text { and } \\
a_{i}+a_{j} \geq a_{d}+a_{i+j-d}, & \text { if } i+j \geq d .
\end{aligned}
$$

It can be realized by the revlex order with $t_{0}<t_{1}<\cdots<t_{d}$ or by the revlex order with $t_{0}>t_{1}>\cdots>t_{d}$. The corresponding initial ideal of $P$ is $\left(t_{1}, \ldots, t_{d-1}\right)^{2}$. The lex-segment ideals $L$ belonging to the cone are characterized by the fact that $L^{2}=\left(x^{d}, y^{a_{d}}\right) L$, that is, they are exactly the lex-segment ideals with a monomial minimal reduction and the reduction number 1 . It is not difficult to show that the simple homogeneous integrally closed ideals of $K[x, y]$ are exactly the ideals of the form $\overline{\left(x^{d}, y^{c}\right)}$, with $\operatorname{GCD}(d, c)=1$. In other words, $\overline{C(\mathbf{i})}$ contains (the exponent vectors of) all the simple integrally closed ideals of order $d$. The associated permutation cone is $C_{\sigma}$ with $\sigma=(d, d-1, \ldots, 1)$. For $d \leq 3$ one has $C(\mathbf{i})=C_{\sigma}$. For $d=4$ one has $C$ (i) $\supsetneq C_{\sigma}$ and $\overline{C(\mathbf{i})}=\overline{C_{\sigma}} \cup \overline{C_{\tau}}$ with $\tau=(4,2,3,1)$. For $d>4$ the cone $\overline{C(\mathbf{i})}$ is not the union of the closure of the permutation cones it contains. For $d=5$, for example, the cone $\overline{C(\mathbf{i})}$ is described by the inequalities

$b_{1}+b_{2} \geq b_{3}+b_{4}, \quad b_{2}+b_{3} \geq b_{4}+b_{5}, \quad b_{1} \geq b_{i} \geq b_{5}$ with $i=2,3,4$,

and hence it intersects but it does not contain the cone associated with the permutation $(5,2,4,3,1)$.

(3) Apart from the example discussed in (1), the other Cohen-Macaulay monomial initial ideals of $P$ arising from lex orders are exactly those associated to sequences $\mathbf{i}=(0,1, \ldots, \widehat{j}, \ldots, d)$ for some $0<j<d$. Apart from the example discussed in (2), the other Cohen-Macaulay monomial initial ideals of $P$ arising from revlex orders are exactly those associated to sequences $\mathbf{i}=(0, j, d)$ for some $0<j<d$. Therefore, starting from $d=5$, there are Cohen-Macaulay monomial initial ideals of $P$ not coming from lex or revlex orders. For instance, the initial ideal associated to $\mathbf{i}=(0,1,4,5)$ is such an example.

\section{Describing the Hilbert series of $\operatorname{gr}_{L}(R)$}

Let $L$ be a lex-segment ideal in $R=K[x, y]$ with associated $a$-sequence $a=$ $\left(a_{0}, a_{1}, \ldots, a_{d}\right)$. We have seen in the proof of Theorem 4.13 that the multiplicity $e_{0}(L)$ can be expressed as a linear function in $a_{i}$ 's. In terms of initial ideals of $P$, that assertion can be rephrased as follows. Let $I$ be a monomial initial ideal of $P$ 
and let $C_{I}$ be the corresponding closed cone in the Gröbner fan of $P$,

$$
C_{I}=\left\{a \in \mathbb{Q}_{\geq 0}^{d+1}: \operatorname{in}_{\tau}\left(\operatorname{in}_{a}(P)\right)=I\right\}
$$

where $\tau$ is a given term order such that $\operatorname{in}_{\tau}(P)=I$. Let $\mathbf{i}=\left(0=i_{0}<i_{1}<\ldots<i_{k}=d\right)$ and let the set of the integers $0 \leq j \leq d$ be such that $t_{j} \notin \sqrt{I}$. Then

$$
\sqrt{I}=\left(t_{j}: j \notin \mathbf{i}\right)+\left(t_{i_{v}} t_{i_{r}}: r-v>1\right) .
$$

Consider the linear form in $\mathbb{Z}\left[A_{0}, \ldots, A_{d}\right]$ given by

$$
e_{0}^{I}=A_{0}\left(i_{1}-i_{0}\right)+\sum_{t=1}^{k-1} A_{i_{t}}\left(i_{t+1}-i_{t-1}\right)+A_{i_{k}}\left(i_{k}-i_{k-1}\right),
$$

where the $A_{i}$ are variables. For every lex-segment ideal $L$ with the associated sequence $a$ belonging to $C_{I}$ one has that $e_{0}(L)$ is equal to $e_{0}^{I}$ evaluated at $A=a$.

So the "same" formula holds in all the cones of the Gröbner fan associated with the same radical, that is, in all the cones of which the union forms a maximal cone of the secondary fan [Sturmfels 1996, p. 71]. We establish now similar formulas for the Hilbert function $H^{1}(L, k)$ and the $h$-polynomial of $\operatorname{gr}_{L}(R)$.

To this end, consider $S=K\left[t_{0}, t_{1}, \ldots, t_{d}\right]$ equipped with its natural $\mathbb{Z}^{d+1}$-graded structure. The quotient $S / I$ is $\mathbb{Z}^{d+1}$-graded and we denote by $H_{S / I}(\underline{t})$ its $\mathbb{Z}^{d+1}$ graded Hilbert series, namely

$$
H_{S / I}(\underline{t})=\sum_{\alpha \in \mathbb{N}^{d+1}} \operatorname{dim}[S / I]_{\alpha} t^{\alpha}=\sum_{t^{\alpha} \notin I} t^{\alpha}
$$

where $t^{\alpha}=t_{0}^{\alpha_{0}} \cdots t_{d}^{\alpha_{d}}$. The key observation is contained in the following lemma.

Lemma 6.1. Let $L$ be a lex-segment ideal with associated vector a belonging to $C_{I}$. For $k \in \mathbb{N}$ set $M_{k}(I)=\left\{\alpha \in \mathbb{N}^{d+1}: t^{\alpha} \notin I,|\alpha|=k\right\}$. Denote by $\sum M_{k}(I)$ the sum of the vectors in $M_{k}(I)$. By construction $\sum M_{k}(I) \in \mathbb{N}^{d+1}$ and

$$
H^{1}(L, k-1)=a \cdot \sum M_{k}(I)
$$

for all $k$.

Proof. Set $C_{k}=a \cdot \sum M_{k}(I)$. Writing $t^{\alpha}$ as $t_{j_{1}} \cdots t_{j_{k}}$, we may rewrite $C_{k}$ as the sum $a_{j_{1}}+\cdots+a_{j_{k}}$ over all monomials $t_{j_{1}} \cdots t_{j_{k}} \notin I$. By construction

$a_{j_{1}}+\cdots+a_{j_{k}}=\min \left\{a_{i_{1}}+a_{i_{2}}+\cdots+a_{i_{k}}: i_{1}+i_{2}+\cdots+i_{k}=j_{1}+j_{2}+\cdots+j_{k}\right\}$

if and only if $t_{j_{1}} \cdots t_{j_{k}} \notin I$. Therefore $C_{k}$ is the sum over all $v, 0 \leq v \leq k d$ of $\min \left\{a_{i_{1}}+a_{i_{2}}+\cdots+a_{i_{k}}: i_{1}+i_{2}+\cdots+i_{k}=v\right\}$. But this is exactly $H^{1}(L, k-1)$; see Lemma 3.5.

In terms of Hilbert series Lemma 6.1 can be rewritten as the follows. 
Lemma 6.2. Let $L$ be a monomial ideal with associated sequence a belonging to $C_{I}$. Then

$$
H_{L}^{1}(z)=a \cdot \nabla H_{S / I}(\underline{t})_{t_{i}=z}
$$

where $\nabla=\left(\partial / \partial t_{0}, \ldots, \partial / \partial t_{d}\right)$ is the gradient operator.

Remark 6.3. (1) The series $H_{S / I}(\underline{t})$ is rational and it can be described in terms of the multigraded Betti numbers $\beta_{i, \alpha}(S / I)=\operatorname{dim}_{K} \operatorname{Tor}_{i}^{S}(S / I, K)_{\alpha}$ as

$$
H_{S / I}(\underline{t})=\frac{\sum_{i, \alpha}(-1)^{i} t^{\alpha} \beta_{i, \alpha}(S / I)}{\Pi_{i=0}^{d}\left(1-t_{i}\right)} .
$$

(2) A rational expression of $H_{S / I}(t)$ can be computed also from a Stanley decomposition of $S / I$. For a monomial ideal $I$ a Stanley decomposition of $S / I$ is a finite set $\Omega$ of pairs $(\sigma, \tau)$ where $\sigma \in \mathbb{N}^{d+1}$ and $\tau \subseteq\{0, \ldots, d\}$ which induces a decomposition

$$
S / I=\bigoplus_{(\sigma, \tau) \in \Omega} t^{\sigma} K\left[t_{i}: i \in \tau\right]
$$

as a $K$-vector space. Stanley decompositions always exist but they are far from being unique. There are algorithms to compute them; see [Maclagan and Smith 2005] for more. For every Stanley decomposition $\Omega$ of $S / I$ clearly one has

$$
H_{S / I}(\underline{t})=\sum_{(\sigma, \tau) \in \Omega} \frac{t^{\sigma}}{\Pi_{i \in \tau}\left(1-t_{i}\right)} .
$$

Combining Lemma 6.1, Remark 6.3 with Lemma 6.2 we obtain:

Corollary 6.4. For every cone $C_{I}$ and for a monomial ideal $L$ whose associated sequence a belongs to $C_{I}$ we have

$$
H_{L}^{1}(z)=|a| \frac{1+(d-1) z}{(1-z)^{3}}+a \cdot \sum_{i \geq 1}(-1)^{i} \sum_{\alpha} \beta_{i, \alpha}(S / I) \frac{z^{|\alpha|-1}}{(1-z)^{d+1}} \alpha,
$$

where $\beta_{i, \alpha}(S / I)$ are the multigraded Betti numbers of $S / I$. Moreover

$$
H_{L}^{1}(z)=a \cdot \sum_{(\sigma, \tau) \in \Omega} \frac{z^{|\sigma|-1}}{(1-z)^{|\tau|+1}}(z \tau+(1-z) \sigma),
$$

where $\Omega$ is a Stanley decomposition of $S / I$ and we have identified the subset $\tau$ with the corresponding 0/1-vector.

The next proposition summarizes what we have proved so far concerning formulas for $h^{I}$ and related invariants.

Proposition 6.5. Given a cone $C_{I}$ of the Gröbner fan of $P$ there are polynomials $h^{I} \in \mathbb{Z}[A, z]$ and $Q^{I}, Q_{1}^{I} \in \mathbb{Q}[A, z]$ linear in the variables $A=A_{0}, \ldots, A_{d}$ and without constant term, such that 
(1) for every monomial ideal $L$ with associated sequence a belonging to $C_{I}$, the polynomial $h^{I}$ evaluated at $A=$ a equals the $h$-polynomial of $L, Q^{I}$ evaluated at $A=$ a equals the Hilbert polynomial $P_{L}$ of $L$, and $Q_{1}^{I}$ evaluated at $A=a$ equals the Hilbert polynomial $P_{L}^{1}$ of $L$;

(2) $h^{I}, Q^{I}$ and $Q_{1}^{I}$ can be expressed in terms of the multigraded Betti numbers of $I$; they can also be expressed in terms of a Stanley decomposition of $S / I$;

(3) in particular,

$$
h^{I}=|A|(1+(d-1) z)+A \sum_{i \geq 1}(-1)^{i} \sum_{\alpha} \beta_{i, \alpha}(S / I) \frac{z^{|\alpha|-1}}{(1-z)^{d-2}} \alpha,
$$

where $\beta_{i, \alpha}(S / I)$ are the multigraded Betti numbers of $S / I$, and

$$
h^{I}=A \cdot \sum_{(\sigma, \tau) \in \Omega} z^{|\sigma|-1}(1-z)^{2-|\tau|}(z \tau+(1-z) \sigma),
$$

where $\Omega$ is a Stanley decomposition of $S / I$; explicit expressions for $Q^{I}$ and $Q_{1}^{I}$ can be obtained from that of $h^{I}$.

Similarly one has expressions for the Hilbert coefficients $e_{i}^{I}$ as a linear function in the variables $A$. Now we discuss the dependence of the polynomials $h^{I}$ and $Q_{1}^{I}$ on $I$.

Proposition 6.6. Let $I, J$ be monomial initial ideals of $P$. Then

(1) $e_{0}^{I}=e_{0}^{J}$ if and only if $\sqrt{I}=\sqrt{J}$,

(2) $h^{I}=h^{J}$ if and only if $I=J$, and

(3) $Q_{1}^{I}=Q_{1}^{J}$ if and only if I and $J$ have the same saturation, equivalently, they coincide from a certain degree on.

Proof. Denote by $A$ the vector of variables $\left(A_{0}, \ldots, A_{d}\right)$. We have discussed already the fact that the formula for the multiplicity $e_{0}^{I}$ identifies and it is identified by the radical of $I$. For statement (2), we have already seen that the coefficient $C_{k}$ of $z^{k}$ in the series $h^{I} /(1-z)^{3}$ is exactly $A \cdot \sum M_{k+1}(I)$. Hence $h^{I}=h^{J}$ holds if and only if $A \cdot \sum M_{k}(I)=A \cdot \sum M_{k}(J)$ for all $k$, that is, $\sum M_{k}(I)=\sum M_{k}(J)$ as vectors for every $k$. By virtue of [Sturmfels 1996, Corollary 2.7], we conclude that $h^{I}=h^{J}$ implies $I=J$. For (3) one just applies the same argument to all large degrees.

For an ideal $I$ of dimension $v$ we denote by $I^{t o p}$ the component of dimension $v$ of $I$, that is, the intersection of the primary components of $I$ of dimension $v$.

Remark 6.7. Let $I, J$ be monomial initial ideals of $P$. In terms of $M_{k}(I)$ the condition $Q^{I}=Q^{J}$ is equivalent to $\sum M_{k}(I)-\sum M_{k-1}(I)=\sum M_{k}(J)-\sum M_{k-1}(J)$ for all $k \gg 0$. There is some computational evidence that $Q^{I}=Q^{J}$ could be 
equivalent to $I^{t o p}=J^{t o p}$. This is related with the hypergeometric Gröbner fan of $P$; see [Saito et al. 2000, Section 3.3]. In particular Example 3.3.7 in [Saito et al. 2000] discusses the secondary fan, the hypergeometric fan and Gröbner fan of $P$ for $d=4$.

\section{The big Cohen-Macaulay cone}

Starting from $d=3$, the Cohen-Macaulay region $C M_{d}$ is not a cone, that is to say it is not convex; see Section 8 for examples. However, a bunch of the cones $C$ (i) get together to form a big cone.

Proposition 7.1. Let $B_{d}=\cup_{\mathbf{i}} \overline{C(\mathbf{i})}$ where the union is extended to all the sequences $\mathbf{i}=\left\{0=i_{0}<i_{1}<\cdots<i_{k}=d\right\}$ such that $i_{v}-i_{v-1} \leq 2$ for all $v=1, \ldots, k$. Then $B_{d}$ is the closed cone described in terms of the $b_{i}$ 's by the inequalities $b_{j} \leq b_{j+2}$ for all $j=1, \ldots, d-2$.

Proof. Let $B^{\prime}$ be the cone described by the inequalities $b_{j} \leq b_{j+2}$ for all $j=$ $1, \ldots, d-2$. We have to show that $B_{d}=B^{\prime}$. For the inclusion $\subseteq$, let $a \in B_{d}$ and $b_{j}=a_{j}-a_{j-1}$. Then $a \in \overline{C(\mathbf{i})}$ for a sequence $\mathbf{i}=\left\{0=i_{0}<i_{1}<\cdots<i_{k}=d\right\}$ such that $i_{v}-i_{v-1} \leq 2$ for all $v=1, \ldots, k$. For every $j, 1 \leq j \leq d-2$, at least one among $j$ and $j+1$ is in $\mathbf{i}$. We distinguish two cases.

Case 1. $j \in \mathbf{i}$, say $j=i_{v}$. Then set $s=j-1$ and $r=j+2$. We have $2 i_{v} \leq s+r \leq$ $i_{v}+i_{v+1}$ and so, by Theorem 4.11 (5), $a_{s}+a_{r} \geq a_{i_{v}}+a_{s+r-i_{v}}$ is one of the defining inequalities of $\overline{C(\mathbf{i})}$. Explicitly, $a_{j-1}+a_{j+2} \geq a_{j}+a_{j+1}$, that is, $b_{j} \leq b_{j+2}$.

Case 2. $j+1 \in \mathbf{i}$, say $j+1=i_{v+1}$. Then set $s=j-1$ and $r=j+2$. We have $i_{v}+i_{v+1} \leq s+r \leq 2 i_{v+1}$ and so, by Theorem 4.11 (5), $a_{s}+a_{r} \geq a_{i_{v+1}}+a_{s+r-i_{v+1}}$ is one of the defining inequalities of $\overline{C(\mathbf{i})}$. Explicitly, $a_{j-1}+a_{j+2} \geq a_{j}+a_{j+1}$, that is, $b_{j} \leq b_{j+2}$.

For the inclusion $\supseteq$, let $a \in B^{\prime}$ and $b_{i}=a_{i}-a_{i-1}$. Set

$$
U=\left\{j: 1 \leq j \leq d-1, b_{j}>b_{j+1}\right\} .
$$

Since $b_{j} \leq b_{j+2}$ for all $j, U$ does not contain pairs of consecutive numbers. Set $\mathbf{i}=\{0,1, \ldots, d\} \backslash U=\left(0=i_{0}<\cdots<i_{k}=d\right)$. Note that for all $0 \leq r, s \leq d$ such that $s-r \geq 2$ one has $b_{s}+b_{s-1} \geq b_{r+1}+b_{r}$ and hence $a_{s}+a_{r} \geq a_{s-2}+a_{r+2}$. This fact, together with the definition of $U$, implies that for all $0 \leq r, s \leq d$ one has

$$
a_{s}+a_{r} \geq \begin{cases}a_{j}+a_{j+1}, & \text { if } s+r=2 j+1, \\ 2 a_{j}, & \text { if } s+r=2 j \text { and } j \in \mathbf{i}, \\ a_{j-1}+a_{j+1}, & \text { if } s+r=2 j \text { and } j \notin \mathbf{i} .\end{cases}
$$

Using this information one proves directly that $a$ satisfies the inequalities in Theorem 4.11 (5) defining $\overline{C(\mathbf{i})}$. 
Remark 7.2. (1) The number $f_{d}$ of the cones $\overline{C(\mathbf{i})}$ appearing in the description of $B_{d}$ satisfies the recursion $f_{d}=f_{d-1}+f_{d-2}$ with $f_{1}=1$ and $f_{2}=2$. Hence $f_{d}$ is the $(d+1)$-th Fibonacci number.

(2) One also has $B_{d}=\cup \overline{C_{\sigma}}$ where $\sigma \in S_{d}$ satisfies $\sigma(j)<\sigma(j+2)$ for $j=$ $1, \ldots, d-2$. There are $\left(\begin{array}{c}d \\ \lfloor d / 2\rfloor\end{array}\right)$ such permutations.

(3) Indeed, each cone $\overline{C(\mathbf{i})}$ appearing in the description of $B_{d}$ is the union of permutation cones $\overline{C_{\sigma}}$. Precisely, the permutations involved are those $\sigma \in S_{d}$ such that $\sigma(j)<\sigma(j+2)$ for all $j=1, \ldots, d-2$ and such that $\sigma(j)>\sigma(j+1)$ if and only if $j \notin \mathbf{i}$. The number of these permutations, say $n(\mathbf{i})$, is a product of Catalan numbers. Recall that the $n$-th Catalan number is $c(n)=(n+1)^{-1}\left(\begin{array}{c}2 n \\ n\end{array}\right)$. Decompose $\{1, \ldots, d\} \backslash \mathbf{i}$ as a disjoint union $\cup_{i=1}^{t} V_{i}$ where $V_{i}$ are of the form $\{a, a+2, \ldots\}$ and are maximal. Then $n(\mathbf{i})=c\left(\left|V_{1}\right|\right) \cdots c\left(\left|V_{t}\right|\right)$. For instance, if $\mathbf{i}=(0,2,3,5,7,9,10,12,14)$ then $\{1, \ldots, 14\} \backslash \mathbf{i}=\{1\} \cup\{4,6,8\} \cup\{11,13\}$ and hence $\overline{C(\mathbf{i})}$ is the union of $c(1) c(3) c(2)=10$ permutation cones.

(4) The family $B_{d}$ with $d \in \mathbb{N}$ is closed under multiplication, that is, if $a \in B_{d}$ and $a^{\prime} \in B_{e}$ and $\mathbf{c}=a \cdot a^{\prime}$, then $\mathbf{c} \in B_{d+e}$. Set $\mathbf{c}=\left(c_{0}, c_{1}, \ldots, c_{d+e}\right)$. To show that $\mathbf{c} \in B_{d+e}$ one has to prove that $c_{j+2}-c_{j+1} \geq c_{j}-c_{j-1}$, that is,

$$
c_{j+2}+c_{j-1} \geq c_{j+1}+c_{j} .
$$

By definition, $c_{j+2}=a_{v}+a_{u}^{\prime}$ with $v+u=j+2$ and $c_{j-1}=a_{w}+a_{z}^{\prime}$ with $w+z=j-1$. Since $(v-w)+(u-z)=(v+u)-(w+z)=3$ we may assume that $v-w \geq 2$. Then $a_{v}+a_{w} \geq a_{v-2}+a_{w+2}$ and hence

$$
c_{j+2}+c_{j-1}=a_{v}+a_{u}^{\prime}+a_{w}+a_{z}^{\prime} \geq a_{v-2}+a_{u}^{\prime}+a_{w+2}+a_{z}^{\prime} \geq c_{j}+c_{j+1} .
$$

\section{Examples with small $d$}

In this section we describe, for small $d$, the Gröbner fan and the Cohen-Macaulay region, and give formulas for the Hilbert series associated to the various cones. For simplicity, the cones will be described in terms of $b_{1}, \ldots, b_{d}$ where $b_{i}=a_{i}-a_{i-1}$.

For $d=1$, there is not much to say. The ideal $P$ is $0, C M_{1}=\mathbb{Q}_{\geq 0}^{2}$ and

$$
C M_{1} \cap W_{1}=\left\{(0, a) \in \mathbb{N}^{2}: a>0\right\} .
$$

For $d=2$ the Gröbner fan has two maximal cones, both Cohen-Macaulay. The lex cone $C(0,1,2)$ is described by $b_{1} \leq b_{2}$ and the revlex cone $C(0,2)$ is described by $b_{1} \geq b_{2}$.

For $d=3$ the Gröbner fan has 8 maximal cones. 4 of them are Cohen-Macaulay and 4 have depth 1 . We show below the cones. Each table shows

(1) an initial ideal $I$ of $P$, 
(2) the linear inequalities defining the corresponding cone in the Gröbner fan, and

(3) the coefficients of the $h$-vector of $\operatorname{gr}_{L}(R)$ for the ideal $L$ corresponding to points $\left(a_{0}, a_{1}, a_{2}, a_{3}\right)$ in the cone.

The expressions of the $h$-vectors have been computed using Stanley decompositions and the formula in Corollary 6.4. The Stanley decompositions have been computed by the algorithm presented in [Maclagan and Smith 2005]. Cones (a), (b), (c) and (d) are Cohen-Macaulay cones. In particular (a) is the lex cone and (d) is the revlex cone. The union of (a), (b) and (c) is the big cone $B_{3}$ and it is defined by $b_{1} \leq b_{3}$. The revlex cone (d) is isolated; it intersects $B_{3}$ only at $b_{1}=b_{2}=b_{3}$. In particular the Cohen-Macaulay region is not a cone.

\begin{tabular}{|l|}
\hline$(a)\left(t_{1} t_{3}, t_{0} t_{3}, t_{0} t_{2}\right)$, \\
$b_{1} \leq b_{2} \leq b_{3}$, \\
$\left(h_{0}\right) a_{0}+a_{1}+a_{2}+a_{3}$ \\
$\left(h_{1}\right) a_{1}+a_{2}$ \\
\hline$(c)\left(t_{2}^{2}, t_{0} t_{3}, t_{0} t_{2}\right)$, \\
$b_{1} \leq b_{3} \leq b_{2}$ \\
$\left(h_{0}\right) a_{0}+a_{1}+a_{2}+a_{3}$ \\
$\left(h_{1}\right) 2 a_{1}-a_{2}+a_{3}$ \\
\hline
\end{tabular}

\begin{tabular}{|l|}
\hline$(b)\left(t_{1} t_{3}, t_{0} t_{3}, t_{1}^{2}\right)$ \\
$b_{2} \leq b_{1} \leq b_{3}$ \\
$\left(h_{0}\right) a_{0}+a_{1}+a_{2}+a_{3}$ \\
$\left(h_{1}\right) a_{0}-a_{1}+2 a_{2}$ \\
\hline$(d)\left(t_{2}^{2}, t_{1} t_{2}, t_{1}^{2}\right)$, \\
$b_{3} \leq b_{2} \leq b_{1}$ \\
$\left(h_{0}\right) a_{0}+a_{1}+a_{2}+a_{3}$ \\
$\left(h_{1}\right) 2 a_{0}-a_{1}-a_{2}+2 a_{3}$ \\
\hline
\end{tabular}

The non-Cohen-Macaulay cones are

\begin{tabular}{|l|}
\hline$(e)\left(t_{2}^{2}, t_{1} t_{2}, t_{0} t_{2}, t_{0}^{2} t_{3}\right)$, \\
$b_{3} \leq b_{1} \leq b_{2}$ and $b_{3}+b_{2} \geq 2 b_{1}$, \\
$\left(h_{0}\right) a_{0}+a_{1}+a_{2}+a_{3}$ \\
$\left(h_{1}\right) a_{0}+a_{1}-2 a_{2}+2 a_{3}$ \\
$\left(h_{2}\right)-a_{0}+a_{1}+a_{2}-a_{3}$ \\
\hline$(g)\left(t_{1} t_{3}, t_{1} t_{2}, t_{1}^{2}, t_{2}^{3}\right)$ \\
$b_{2} \leq b_{3} \leq b_{1}$ and $b_{1}+b_{2} \geq 2 b_{3}$, \\
$\left(h_{0}\right) a_{0}+a_{1}+a_{2}+a_{3}$ \\
$\left(h_{1}\right) 2 a_{0}-2 a_{1}+a_{2}+a_{3}$ \\
$\left(h_{2}\right) a_{1}-2 a_{2}+a_{3}$ \\
\hline
\end{tabular}

\begin{tabular}{|l|}
\hline$(f)\left(t_{1} t_{3}, t_{1} t_{2}, t_{1}^{2}, t_{0} t_{3}^{2}\right)$, \\
$b_{2} \leq b_{3} \leq b_{1}$ and $b_{1}+b_{2} \leq 2 b_{3}$, \\
$\left(h_{0}\right) a_{0}+a_{1}+a_{2}+a_{3}$ \\
$\left(h_{1}\right) 2 a_{0}-2 a_{1}+a_{2}+a_{3}$ \\
$\left(h_{2}\right)-a_{0}+a_{1}+a_{2}-a_{3}$ \\
\hline$(h)\left(t_{2}^{2}, t_{1} t_{2}, t_{0} t_{2}, t_{1}^{3}\right)$ \\
$b_{3} \leq b_{1} \leq b_{2}$ and $b_{3}+b_{2} \leq 2 b_{1}$, \\
$\left(h_{0}\right) a_{0}+a_{1}+a_{2}+a_{3}$ \\
$\left(h_{1}\right) a_{0}+a_{1}-2 a_{2}+2 a_{3}$ \\
$\left(h_{2}\right) a_{0}-2 a_{1}+a_{2}$ \\
\hline
\end{tabular}

For $d=4$ there are 42 cones of the Gröbner fan. 10 of them have depth 0 , 24 have depth 1 and 8 are Cohen-Macaulay. The big Cohen-Macaulay cone is the union of 5 of the 8 Cohen-Macaulay cones. The remaining 3 are isolated. The following example illustrates Proposition 6.6 and Remark 6.7. The ideals $I, J$ below are non-Cohen-Macaulay initial ideals of $P$. They satisfy $Q^{I}=Q^{J}$ and $Q_{1}^{I} \neq Q_{1}^{J}$. We display the ideals and the formulas for the coefficients $e_{0}, e_{1}, e_{2}$ 
that have been computed via Stanley decompositions.

\begin{tabular}{|l|}
$I\left(t_{1} t_{3}, t_{1} t_{2}, t_{0} t_{2}, t_{3}^{3}, t_{1}^{2} t_{4}, t_{1}^{3}, t_{2} t_{4}, t_{2} t_{3}, t_{2}^{2}\right)$ \\
$\left(e_{0}\right) 4 a_{0}+4 a_{4}$ \\
$\left(e_{1}\right) 3 a_{0}-a_{1}-3 a_{3}+4 a_{4}$ \\
$\left(e_{2}\right)-a_{0}+2 a_{1}-2 a_{3}+a_{4}$
\end{tabular}

$J\left(t_{1} t_{3}, t_{1} t_{2}, t_{1}^{2}, t_{3}^{3}, t_{2} t_{4}, t_{2} t_{3}, t_{2}^{2}\right)$
$\left(e_{0}\right) 4 a_{0}+4 a_{4}$
$\left(e_{1}\right) 3 a_{0}-a_{1}-3 a_{3}+4 a_{4}$
$\left(e_{2}\right) a_{2}-2 a_{3}+a_{4}$

In this case $I^{t o p}=J^{t o p}=\left(t_{1} t_{3}, t_{2}, t_{3}^{3}, t_{1}^{2}\right)$ as expected by Remark 6.7 and $J=$ $J^{s a t} \neq I^{s a t}=\left(t_{2}, t_{1} t_{3}, t_{1}^{2} t_{4}, t_{3}^{3}, t_{1}^{3}\right)$ as proved in Proposition 6.6.

\section{Acknowledgements}

We thank Jürgen Herzog, Rekha Thomas, Greg Smith and Bernd Sturmfels for useful discussions and references related to the topic of the present paper. Most of the results of this paper have been discovered and confirmed by extensive computations with the computer algebra program CoCoA, which is available at http:// cocoa.dima.unige.it.

\section{References}

[Bruns and Conca 2003] W. Bruns and A. Conca, "Gröbner bases and determinantal ideals", pp. 9-66 in Commutative algebra, singularities and computer algebra (Sinaia, Romania, 2002), edited by J. Herzog and V. Vuletescu, NATO Sci. Ser. II Math. Phys. Chem. 115, Kluwer Acad. Publ., Dordrecht, 2003. MR 2005a:13025 Zbl 1052.13010

[Bruns and Herzog 1993] W. Bruns and J. Herzog, Cohen-Macaulay rings, Cambridge Studies in Advanced Mathematics 39, Cambridge University Press, Cambridge, 1993. MR 95h:13020 Zbl 0788.13005

[Conca et al. 2005] A. Conca, E. De Negri, A. V. Jayanthan, and M. E. Rossi, "Graded rings associated with contracted ideals", J. Algebra 284:2 (2005), 593-626. MR 2005k:13006 Zbl 1098.13007

[D’Cruz et al. 1999] C. D'Cruz, K. N. Raghavan, and J. K. Verma, “Cohen-Macaulay fiber cones”, pp. 233-246 in Commutative algebra, algebraic geometry, and computational methods (Hanoi, 1996), edited by D. Eisenbud, Springer, Singapore, 1999. MR 2000i:13003 Zbl 0945.13015

[Delfino et al. 2003] D. Delfino, A. Taylor, W. V. Vasconcelos, N. Weininger, and R. H. Villarreal, "Monomial ideals and the computation of multiplicities", pp. 87-106 in Commutative ring theory and applications (Fez, Morocco, 2001), edited by M. Fontana et al., Lecture Notes in Pure and Appl. Math. 231, Dekker, New York, 2003. MR 2005g:13015 Zbl 1066.13004

[Eisenbud 1995] D. Eisenbud, Commutative algebra, Graduate Texts in Mathematics 150, Springer, New York, 1995. MR 97a:13001 Zbl 0819.13001

[Eisenbud and Goto 1984] D. Eisenbud and S. Goto, "Linear free resolutions and minimal multiplicity”, J. Algebra 88:1 (1984), 89-133. MR 85f:13023 Zbl 0531.13015

[Hoşten and Thomas 2003] S. Hoşten and R. R. Thomas, "Gomory integer programs", Math. Program. 96:2, Ser. B (2003), 271-292. MR 2004f:90087 Zbl 1082.90068

[Huckaba and Marley 1993] S. Huckaba and T. Marley, "Depth properties of Rees algebras and associated graded rings”, J. Algebra 156:1 (1993), 259-271. MR 94d:13006 Zbl 0813.13010 
[Huneke 1989] C. Huneke, "Complete ideals in two-dimensional regular local rings", pp. 325-338 in Commutative algebra (Berkeley, CA, 1987), edited by M. Hochster et al., Math. Sci. Res. Inst. Publ. 15, Springer, New York, 1989. MR 90i:13020 Zbl 0732.13007

[Huneke and Swanson 2006] C. Huneke and I. Swanson, Integral closure of ideals, rings, and modules, London Mathematical Society Lecture Note Series 336, Cambridge University Press, Cambridge, 2006. MR 2266432 Zbl 1117.13001

[Lipman and Teissier 1981] J. Lipman and B. Teissier, "Pseudorational local rings and a theorem of Briançon-Skoda about integral closures of ideals", Michigan Math. J. 28:1 (1981), 97-116. MR 82f:14004 Zbl 0464.13005

[Maclagan and Smith 2005] D. Maclagan and G. G. Smith, "Uniform bounds on multigraded regularity", J. Algebraic Geom. 14:1 (2005), 137-164. MR 2005g:14098 Zbl 1070.14006

[O'Shea and Thomas 2005] E. O'Shea and R. R. Thomas, “Toric initial ideals of $\Delta$-normal configurations: Cohen-Macaulayness and degree bounds", J. Algebraic Combin. 21:3 (2005), 247-268. MR 2006c:13031 Zbl 1082.13017

[Saito et al. 2000] M. Saito, B. Sturmfels, and N. Takayama, Gröbner deformations of hypergeometric differential equations, Algorithms and Computation in Mathematics 6, Springer, Berlin, 2000. MR 2001i:13036 Zbl 0946.13021

[Sturmfels 1996] B. Sturmfels, Gröbner bases and convex polytopes, University Lecture Series 8, American Mathematical Society, Providence, RI, 1996. MR 97b:13034 Zbl 0856.13020

[Trung and Hoa 1986] N. V. Trung and L. T. Hoa, "Affine semigroups and Cohen-Macaulay rings generated by monomials", Trans. Amer. Math. Soc. 298:1 (1986), 145-167. MR 87j:13032 Zbl 0631.13020

[Valla 1979] G. Valla, "On form rings which are Cohen-Macaulay”, J. Algebra 58:2 (1979), 247250. MR 80h:13025 Zbl 0428.13010

[Verma 1991] J. K. Verma, "Rees algebras of contracted ideals in two-dimensional regular local rings", J. Algebra 141:1 (1991), 1-10. MR 92j:13004 Zbl 0736.13016

[Wilf 2002] H. S. Wilf, "The patterns of permutations", Discrete Math. 257:2-3 (2002), 575-583. MR 2003h:05005 Zbl 1028.05002

[Zariski and Samuel 1960] O. Zariski and P. Samuel, Commutative algebra, vol. II, Van Nostrand, Princeton, 1960. MR 22 \#11006 Zbl 0121.27801

Communicated by Craig Huneke

Received 2007-02-01 Revised 2007-08-27 Accepted 2007-09-29

conca@dima.unige.it

denegri@dima.unige.it

rossim@dima.unige.it
Dipartimento di Matematica, Università di Genova, Via Dodecaneso 35, I-16146 Genova, Italy

Dipartimento di Matematica, Università di Genova, Via Dodecaneso 35, I-16146 Genova, Italy

Dipartimento di Matematica, Università di Genova, Via Dodecaneso 35, I-16146 Genova, Italy 


\title{
Swan conductors for $p$-adic differential modules, I: A local construction
}

\author{
Kiran S. Kedlaya
}

\begin{abstract}
We define a numerical invariant, the differential Swan conductor, for certain differential modules on a rigid analytic annulus over a $p$-adic field. This gives a definition of a conductor for $p$-adic Galois representations with finite local monodromy over an equal characteristic discretely valued field, which agrees with the usual Swan conductor when the residue field is perfect. We also establish analogues of some key properties of the usual Swan conductor, such as integrality (the Hasse-Arf theorem), and the fact that the graded pieces of the associated ramification filtration on Galois groups are abelian and killed by $p$.
\end{abstract}

\section{Introduction}

In this paper, we define a numerical invariant, which we call the differential Swan conductor, for certain differential modules on a rigid analytic annulus over a complete nonarchimedean field of mixed characteristics. We then use this definition to define a differential Swan conductor for $p$-adic Galois representations with finite local monodromy over an equal characteristic discretely valued field, whose residue field need not be perfect. The latter will coincide with the usual Swan conductor in the case of a perfect residue field.

The construction of the differential Swan conductor proceeds by measuring the failure of convergence of the Taylor isomorphism, or equivalently, the failure of local horizontal sections for the connection to converge on as large a disc as possible. This phenomenon distinguishes the study of differential equations over $p$ adic fields from its classical analogue, and the relationship with Swan conductors explains the discrepancy in terms of wild ramification in characteristic $p$. (The analogy between irregularity of connections and wild ramification has been known

MSC2000: primary 11S15; secondary 14F30.

Keywords: $p$-adic differential modules, Swan conductors, wild ramification, Hasse-Arf theorem,

imperfect residue fields.

This material was first presented at the Hodge Theory conference at Venice International University in June 2006; that presentation was sponsored by the Clay Mathematics Institute. The author was additionally supported by NSF grant DMS-0400727, NSF CAREER grant DMS-0545904, and a Sloan Research Fellowship. 
for a while, but recent developments have pushed it further, e.g., construction of a de Rham analogue of local $\epsilon$-factors [Beilinson et al. 2002].)

In the case of Galois representations over an equal characteristic discretely valued field with perfect residue field, the differential interpretation of the Swan conductor is known from the work of several authors, including André, Christol and Mebkhout, Crew, Matsuda, Tsuzuki, and others; see [Kedlaya 2005a, Section 5] for an overview. The question of extending this interpretation to the case of imperfect residue field was first raised by Matsuda [2004], who proposed giving a differential interpretation of the logarithmic conductor of [Abbes and Saito 2002; Abbes and Saito 2003]. Our point of view is a bit different: we first construct a numerical invariant from differential considerations, and check that it has good properties. These include the Hasse-Arf property, i.e., integrality of conductors (Theorem 2.8.2), and the fact that the associated ramification filtration on Galois groups has graded pieces which are elementary abelian (Theorem 3.5.13). Only then do we pose questions about reconciling the definition with other constructions; we do not answer any of these.

In a subsequent paper, we will apply this construction to overconvergent $F$ isocrystals on varieties over perfect fields of positive characteristic; in particular, the construction applies to discrete representations of the étale fundamental groups of open varieties. We will pay particular attention to how the differential Swan conductor of a fixed isocrystal changes as we vary the choice of a boundary divisor along which to compute the conductor.

Acknowledgments. The author thanks Liang Xiao for comments on an early draft.

\section{Differential fields}

We start with a summary of some relevant facts about differential fields and modules. We defer to [Kedlaya 2006b, Section 3] (and other explicitly cited references) for more details.

\subsection{Differential modules and twisted polynomials.}

Hypothesis 1.1.1. Throughout this subsection, let $F$ be a differential field of order 1 and characteristic zero, i.e., a field of characteristic zero equipped with a derivation $\partial$.

Definition 1.1.2. Let $F\{T\}$ denote the (noncommutative) ring of twisted polynomials over $F$ [Ore 1933]; its elements are finite formal sums $\sum_{i \geq 0} a_{i} T^{i}$ with $a_{i} \in F$, multiplied according to the rule $T a=a T+\partial(a)$ for $a \in F$.

Remark 1.1.3. The opposite ring of $F\{T\}$ is the ring of twisted polynomials for the differential field given by equipping $F$ with the derivation $-\partial$ instead of $\partial$. 
Definition 1.1.4. A differential module over $F$ is a finite dimensional $F$-vector space $V$ equipped with an action of $\partial$ (subject to the Leibniz rule); any such module inherits a left action of $F\{T\}$ where $T$ acts via $\partial$. For $V$ a differential module over $F$, a cyclic vector in $V$ is a vector $\mathbf{v} \in V$ such that $\mathbf{v}, \partial(\mathbf{v}), \ldots, \partial^{\operatorname{dim}(V)-1}(\mathbf{v})$ form a basis of $V$. A cyclic vector defines an isomorphism $V \cong F\{T\} / F\{T\} P$ of differential modules for some twisted polynomial $P \in F\{T\}$, where the $\partial$-action on $F\{T\} / F\{T\} P$ is left multiplication by $T$.

Lemma 1.1.5. Every differential module over $F$ contains a cyclic vector.

Proof. See, e.g., [Dwork et al. 1994, Theorem III.4.2].

Hypothesis 1.1.6. For the remainder of this subsection, assume that the differential field $F$ is equipped with a nonarchimedean norm $|\cdot|$, and let $V$ denote a nonzero differential module over $F$. Write $v(x)=-\log |x|$ for the valuation corresponding to $|\cdot|$.

Definition 1.1.7. Let $|\partial|_{F}$ denote the operator norm of $\partial$ on $F$. Let $|\partial|_{V \text {,sp }}$ denote the spectral norm of $\partial$ on $V$, i.e., the $\operatorname{limit}_{s \rightarrow \infty}\left|\partial^{s}\right|_{V}^{1 / s}$ for any fixed $F$ compatible norm $|\cdot|_{V}$ on $V$. Any two such norms on $V$ are equivalent [Schneider 2002, Proposition 4.13], so the spectral norm does not depend on the choice. More explicitly, if one chooses a basis of $V$ and lets $D_{s}$ denote the matrix via which $\partial^{s}$ acts on this basis, then

$$
\max \left\{|\partial|_{F, \mathrm{sp}},|\partial|_{V, \mathrm{sp}}\right\}=\max \left\{|\partial|_{F, \mathrm{sp}}, \limsup _{s \rightarrow \infty}\left|D_{S}\right|^{1 / s}\right\},
$$

where the norm applied to $D_{s}$ is the supremum over entries [Christol and Dwork 1994, Proposition 1.3].

Definition 1.1.8. For $P(T)=\sum_{i} a_{i} T^{i} \in F\{T\}$ a nonzero twisted polynomial, define the Newton polygon of $P$ as the lower convex hull of the set $\left\{\left(-i, v\left(a_{i}\right)\right)\right\} \subset$ $\mathbb{R}^{2}$. This Newton polygon obeys the usual additivity rules only for slopes less than $-\log |\partial|_{F}$ [Kedlaya 2006b, Lemma 3.1.5 and Corollary 3.1.6; Robba 1980, Section $1]$.

Proposition 1.1.9 (Christol-Dwork). Suppose $V \cong F\{T\} / F\{T\} P$ and $P$ has least slope $r$. Then

$$
\max \left\{|\partial|_{F},|\partial|_{V, \text { sp }}\right\}=\max \left\{|\partial|_{F}, e^{-r}\right\} .
$$

Proof. See [Christol and Dwork 1994, Théorème 1.5] or [Kedlaya 2006b, Proposition 3.3.7]. 
Proposition 1.1.10 (Robba). Suppose that $F$ is complete for its norm. Then any monic twisted polynomial $P \in F\{T\}$ admits a unique factorization

$$
P=P_{+} P_{m} \cdots P_{1}
$$

such that for some $r_{1}<\cdots<r_{m}<-\log |\partial|_{F}$, each $P_{i}$ is monic with all slopes equal to $r_{i}$, and $P_{+}$is monic with all slopes at least $-\log |\partial|_{F}$.

Proof. This follows by repeated application of Hensel's lemma for twisted polynomials [Robba 1980]; see also [Kedlaya 2006b, Corollary 3.2.4].

\subsection{Differential fields of higher order.}

Hypothesis 1.2.1. Throughout this subsection, let $F$ denote a differential field of order $n$, i.e., a field $F$ equipped with $n$ commuting derivations $\partial_{1}, \ldots, \partial_{n}$. Assume also that $F$ has characteristic zero and is complete for a nonarchimedean norm $|\cdot|$ with corresponding valuation $v$. Let $V$ denote a nonzero differential module over $F$, i.e., a nonzero finite dimensional $F$-vector space equipped with commuting actions of $\partial_{1}, \ldots, \partial_{n}$. We apply the results of the previous subsection by singling out one of $\partial_{1}, \ldots, \partial_{n}$.

Definition 1.2.2. Define the scale of $V$ as

$$
\max \left\{\max \left\{1, \frac{\left|\partial_{i}\right|_{V, \mathrm{sp}}}{\left|\partial_{i}\right|_{F, \mathrm{sp}}}\right\}: i \in\{1, \ldots, n\}\right\}
$$

note that this quantity is at least 1 by definition, with equality at least when $V=F$. For $i=1, \ldots, n$, we say $\partial_{i}$ is dominant for $V$ if $\max \left\{1,\left|\partial_{i}\right|_{V, \mathrm{sp}} /\left|\partial_{i}\right|_{F, \mathrm{sp}}\right\}$ equals the scale of $V$.

Definition 1.2.3. Let $V_{1}, \ldots, V_{m}$ be the Jordan-Hölder factors of $V$ (listed with multiplicity). Define the scale multiset of $V$ as the multiset of cardinality $\operatorname{dim}_{F} V$, consisting of the scale of $V_{j}$ included with multiplicity $\operatorname{dim}_{F} V_{j}$, for $j=1, \ldots, m$. Note that the largest element of the scale multiset equals the scale of $V$.

Remark 1.2.4. If $n=1$ and $V \cong F\{T\} / F\{T\} P$ for $P$ a twisted polynomial, then Proposition 1.1.10 implies that the multiplicity of any $r<-\log |\partial|_{F}$ as a slope of the Newton polygon of $P$ coincides with the multiplicity of $e^{-r} /|\partial|_{F, \text { sp }}$ in the scale multiset of $V$.

Proposition 1.2.5. Suppose that $\left|\partial_{i}\right|_{F} /\left|\partial_{i}\right|_{F, \mathrm{sp}}=s_{0}$ for $i=1, \ldots, n$. Then there is a unique decomposition

$$
V=V_{-} \oplus \bigoplus_{s>s_{0}} V_{s}
$$

of differential modules, such that each Jordan-Hölder factor of $V_{s}$ has scale $s$, and each Jordan-Hölder factor of $V_{-}$has scale at most $s_{0}$. 
Proof. This may be deduced from Proposition 1.1.10, as in [Kedlaya 2006b, Proposition 3.4.3].

Definition 1.2.6. We refer to the decomposition given in Proposition 1.2.5 as the scale decomposition of $V$.

\section{Conductors for $\nabla$-modules}

In this section, we construct the differential Swan conductor for certain differential modules over $p$-adic fields. We will perform all of the calculations under the bifurcated Hypothesis 2.1.3; one of the two options therein allows for nonarchimedean fields which are not discretely valued, but restricts their residue fields, while the other is less restrictive on residue fields, but requires the nonarchimedean norms to be discretely valued.

Notation 2.0.1. For $S$ a set or multiset, write $S^{p}=\left\{s^{p}: s \in S\right\}$. If $A, B$ are two multisets of the same cardinality $d$, then write $A \geq B$ to mean that for $i=1, \ldots, d$, the $i$-th largest element of $A$ is greater than or equal to the $i$-th largest element of $B$ (counting multiplicity).

\subsection{Setup.}

Definition 2.1.1. Given a field $K$ equipped with a (possibly trivial) nonarchimedean norm, for $\rho_{1}, \ldots, \rho_{n} \in(0,+\infty)$, the $\left(\rho_{1}, \ldots, \rho_{n}\right)$-Gauss norm on $K\left[u_{1}, \ldots, u_{n}\right]$ is the norm $|\cdot|_{\rho}$ given by

$$
\left|\sum_{I} c_{I} u_{1}^{i_{1}} \cdots u_{n}^{i_{n}}\right|=\max _{I}\left\{\left|c_{I}\right| \rho_{1}^{i_{1}} \cdots \rho_{n}^{i_{n}}\right\}
$$

this norm extends uniquely to $K\left(u_{1}, \ldots, u_{n}\right)$.

Definition 2.1.2. For $\ell / k$ an extension of fields of characteristic $p>0$, a $p$-basis of $\ell$ over $k$ is a set $B \subset \ell$ with the property that the products $\prod_{b \in B} b^{e_{b}}$, where $e_{b} \in\{0, \ldots, p-1\}$ for all $b \in B$ and $e_{b}=0$ for all but finitely many $b$, are all distinct and form a basis for $\ell$ as a vector space over the compositum $k \ell^{p}$. By a p-basis of $\ell$, we mean a $p$-basis of $\ell$ over $\ell^{p}$.

Hypothesis 2.1.3. For the rest of this section, assume one of the following two sets of hypotheses.

(a) Let $K$ be a field of characteristic zero, complete for a (not necessarily discrete) nonarchimedean norm $|\cdot|$, with residue field $k$ of characteristic $p>0$. Equip $K\left(u_{1}, \ldots, u_{n}\right)$ with the $(1, \ldots, 1)$-Gauss norm. Let $\ell$ be a finite separable extension of $k\left(u_{1}, \ldots, u_{n}\right)$, and let $L$ be the unramified extension with residue field $\ell$ of the completion of $K\left(u_{1}, \ldots, u_{n}\right)$. 
(b) Let $K$ be a field of characteristic zero, complete for a nonarchimedean norm $|\cdot|$, with discrete value group and residue field $k$ of characteristic $p>0$. Let $L$ be an extension of $K$, complete for an extension of $|\cdot|$ with the same value group, whose residue field $\ell$ admits a finite $p$-basis $\bar{B}=\left\{\bar{u}_{1}, \ldots, \bar{u}_{n}\right\}$ over $k$. For $i=1, \ldots, n$, let $u_{i}$ be a lift of $\overline{u_{i}}$ to the valuation ring $\mathfrak{o}_{L}$ of $L$.

Definition 2.1.4. Under either option in Hypothesis 2.1.3, the module of continuous differentials $\Omega_{L / K}^{1}$ is generated by $d u_{1}, \ldots, d u_{n}$; let $\partial_{1}, \ldots, \partial_{n}$ denote the dual basis of derivations (that is, $\partial_{i}=\frac{\partial}{\partial u_{i}}$ ).

Remark 2.1.5. Note that $\left|\partial_{i}\right|_{L} /\left|\partial_{i}\right|_{L, \mathrm{sp}}=|p|^{-1 /(p-1)}$ for $i=1, \ldots, n$, so Proposition 1.2.5 applies.

2.2. Taylor isomorphisms. The scale of a differential module over $L$ can be interpreted as a normalized radius of convergence for the Taylor series, as follows.

Convention 2.2.1. Let $\mathbb{N}_{0}$ denote the monoid of nonnegative integers. For $I \in \mathbb{N}_{0}^{n}$ and $*$ any symbol, we will write $*^{I}$ as shorthand for $*_{1}^{i_{1}} \cdots *_{n}^{i_{n}}$. We also write $I$ ! as shorthand for $i_{1} ! \cdots i_{n} !$.

Definition 2.2.2. Let $V$ be a differential module over $L$. Define the formal Taylor isomorphism on $V$ to be the map $T: V \mapsto V \otimes_{L} L \llbracket x_{1}, \ldots, x_{n} \rrbracket$ given by

$$
T(\mathbf{v})=\sum_{I \in \mathbb{N}_{0}^{n}} \frac{x^{I}}{I !} \partial^{I}(\mathbf{v}) .
$$

We can then interpret the scale of $V$ as the minimum $\lambda$ such that $T$ takes values in $V \otimes_{L} R$, for $R$ the subring of $L \llbracket x_{1}, \ldots, x_{n} \rrbracket$ consisting of series convergent on the open polydisc

$$
\left|x_{i}\right|<\lambda^{-1} \quad(i=1, \ldots, n) .
$$

In particular, if $L^{\prime}$ is a complete extension of $L$, and $x_{1}, \ldots, x_{n} \in L^{\prime}$ satisfy $\left|x_{i}\right|<$ $\lambda^{-1}$ for $\lambda$ the scale of $V$, we obtain by substitution a concrete Taylor isomorphism

$$
T\left(\mathbf{v} ; x_{1}, \ldots, x_{n}\right): V \rightarrow V \otimes_{L} L^{\prime} .
$$

Remark 2.2.3. If $x_{1}, \ldots, x_{n} \in L$ satisfy $\left|x_{i}\right|<1$, then the concrete Taylor isomorphism $T\left(\cdot ; x_{1}, \ldots, x_{n}\right)$ is defined on $L$, and is a $K$-algebra homomorphism carrying $u_{i}$ to $u_{i}+x_{i}$. If $V$ is a differential module of scale $\lambda$, and $\left|x_{i}\right|<\lambda^{-1}$ for $i=1, \ldots, n$, then the concrete Taylor isomorphism $T\left(\cdot ; x_{1}, \ldots, x_{n}\right)$ on $V$ is semilinear over the concrete Taylor isomorphism on $L$.

Remark 2.2.4. Note that $\left|\partial^{I} / I !\right|_{F} \leq 1$ for any $I \in \mathbb{N}_{0}^{n}$. Hence if $x_{1}, \ldots, x_{n} \in L$ satisfy $\left|x_{i}\right|<1$, then for any $f \in L$,

$$
\left|T\left(f ; x_{1}, \ldots, x_{n}\right)-f\right| \leq \max _{i}\left\{\left|x_{i}\right|\right\} \cdot|f| .
$$


In particular, suppose $u_{1}^{\prime}, \ldots, u_{n}^{\prime} \in L$ satisfy $\left|u_{i}^{\prime}\right|=1$, and the images of $u_{1}^{\prime}, \ldots, u_{n}^{\prime}$ in $\ell$ form a $p$-basis of $\ell$ over $k$. Then $T\left(\cdot ; x_{1}, \ldots, x_{n}\right)$ can also be interpreted as the concrete Taylor isomorphism defined with respect to the dual basis of $d u_{1}^{\prime}, \ldots, d u_{n}^{\prime}$ and evaluated at $y_{1}, \ldots, y_{n}$, for $y_{i}=T\left(u_{i}^{\prime} ; x_{1}, \ldots, x_{n}\right)-u_{i}^{\prime}$. This implies that the scale of a differential module computed with respect to (the dual basis to) $d u_{1}, \ldots, d u_{n}$ is no greater than with respect to $d u_{1}^{\prime}, \ldots, d u_{n}^{\prime}$; by the same calculation in reverse, it follows that the two scales are equal. (Francesco Baldassarri has suggested a coordinate-free definition of the scale that explains this remark; we will follow up on this suggestion elsewhere.)

2.3. Frobenius descent. As discovered originally in [Christol and Dwork 1994], in the situations of Hypothesis 2.1.3, one can overcome the limitation on scales imposed by Proposition 1.1.9 by using descent along the substitution $u_{i} \mapsto u_{i}^{p}$.

Definition 2.3.1. Let $V$ be a differential module over $L$ with scale less than $|p|^{-1 /(p-1)}$. If $K$ contains a primitive $p$-th root of unity $\zeta$, we may define an action of the group $(\mathbb{Z} / p \mathbb{Z})^{n}$ on $V$ using concrete Taylor isomorphisms:

$$
\mathbf{v}^{J}=T\left(\mathbf{v} ;\left(\zeta^{j_{1}}-1\right) u_{1}, \ldots,\left(\zeta^{j_{n}}-1\right) u_{n}\right) \quad\left(J \in(\mathbb{Z} / p \mathbb{Z})^{n}\right) .
$$

Let $V_{1}$ be the fixed space under this group action; in particular, taking $V=L$, we obtain a subfield $L_{1}$ of $L$, which we may view as a differential field of order $n$ for the derivations $\partial_{i, 1}=\partial / \partial\left(u_{i}^{p}\right)$. In general, $V_{1}$ may be viewed as a differential module over $L_{1}$, the natural map $V_{1} \otimes_{L_{1}} L \rightarrow V$ is an isomorphism of $L$-vector spaces (by Hilbert 90), and the actions of $\partial_{i}$ and $\partial_{i, 1}$ on $V$ are related by the formula

$$
\partial_{i, 1}=\frac{1}{p u_{i}^{p-1}} \partial_{i}
$$

We call $V_{1}$ the Frobenius antecedent of $V$. If $K$ does not contain a primitive $p$-th root of unity, we may still define the Frobenius antecedent using Galois descent.

Proposition 2.3.2. Let $V$ be a differential module over $L$ with scale $s<|p|^{-1 /(p-1)}$ and scale multiset $S$. Then the scale multiset of the Frobenius antecedent of $V$ is $S^{p}$.

Proof. Since any direct sum decomposition commutes with the formation of the Frobenius antecedent $V_{1}$, it suffices to check that the scale of $V_{1}$ is $s^{p}$. Let $T(\mathbf{v})$ be the formal Taylor isomorphism for $V$, and let $T^{\prime}(\mathbf{v})$ be the formal Taylor isomorphism for $V_{1}$ but with variables $x_{1}^{\prime}, \ldots, x_{n}^{\prime}$.

By [Kedlaya 2005a, Lemma 5.12], for $t, t_{1}$ in any nonarchimedean field,

$$
\left|t-t_{1}\right|<\lambda^{-1}|t| \Longrightarrow\left|t^{p}-t_{1}^{p}\right|<\lambda^{-p}|t|^{p} \quad\left(1<\lambda<|p|^{-1 /(p-1)}\right) .
$$

(We repeat from [Kedlaya 2006b, Lemma 4.4.2] the description of a misprint in the last line of the statement of [Kedlaya 2005a, Lemma 5.12]: one must read 
$r^{1 / p} \rho^{1 / p}, r \rho$ for $r \rho^{1 / p}, r^{p} \rho$, respectively.). Hence the convergence of the isomorphism $T^{\prime}\left(\mathbf{v} ; x_{1}^{\prime}, \ldots, x_{n}^{\prime}\right)$ for $\left|x_{i}^{\prime}\right|<\lambda^{-p}$ implies convergence of $T\left(\mathbf{v} ; x_{1}, \ldots, x_{n}\right)$ for $\left|x_{i}\right|<\lambda^{-1}$, so the scale of $V_{1}$ is at least $s^{p}$. On the other hand, we can obtain $T^{\prime}$ by averaging $T$ over the action of $(\mathbb{Z} / p \mathbb{Z})^{n}$, so the scale of $V_{1}$ is at most $s^{p}$. (Compare [Kedlaya 2005a, Theorem 6.15].)

Remark 2.3.3. It should also be possible to prove Proposition 2.3 .2 by raising both sides of (2.3.1.1) to a large power and comparing the results, but this would appear to be somewhat messy.

Definition 2.3.4. If $V$ is a differential module over $F$ of scale less than

$$
|p|^{-1 /\left(p^{m-1}(p-1)\right)},
$$

by Proposition 2.3.2, we can iterate the construction of a Frobenius antecedent $m$ times; we call the result the $m$-fold Frobenius antecedent of $V$.

Remark 2.3.5. Note that it is also possible to construct antecedents one variable at a time; the point is that since the operators $\partial_{i}, \partial_{j}$ commute for $i \neq j, \partial_{i}$ continues to act on the antecedent with respect to $\partial_{j}$. This will be used in the proof of Proposition 2.5.4.

\section{4. $\nabla$-Modules.}

Notation 2.4.1. Let $\Gamma^{*}$ denote the divisible closure of $\left|K^{*}\right|$. We say a subinterval of $(0,+\infty)$ is aligned if each endpoint at which it is closed belongs to $\Gamma^{*}$.

Remark 2.4.2. One can drop the word "aligned", and all references to $\Gamma^{*}$, everywhere hereafter if one works with Berkovich analytic spaces [Berkovich 1990] instead of rigid analytic spaces. We omit further details.

Notation 2.4.3. For $I$ an aligned interval and $t$ a dummy variable, let $A_{L}(I)$ be the rigid analytic (over $L$ ) subspace of the affine $t$-line over $L$ consisting of points with $|t| \in I$; this space is affinoid if $I$ is closed. (We omit the parentheses if $I$ is described explicitly, e.g., if $I=[\alpha, \beta)$, we write $A_{L}[\alpha, \beta)$ for $A_{L}(I)$.) For $\rho \in I$, we write $|\cdot|_{\rho}$ for the $\rho$-Gauss norm

$$
\left|\sum_{i \in \mathbb{Z}} c_{i} t^{i}\right|_{\rho}=\sup _{i}\left\{\left|c_{i}\right| \rho^{i}\right\} ;
$$

for $\rho \in \Gamma^{*}$, we may interpret $|\cdot|_{\rho}$ as the supremum norm on the affinoid space $A_{L}[\rho, \rho]$.

Lemma 2.4.4. Let $I$ be an aligned interval. For $\rho, \sigma \in I$ and $c \in[0,1]$, put $\tau=\rho^{c} \sigma^{1-c}$. Then for any $f \in \Gamma\left(A_{L}(I), \mathcal{O}\right)$,

$$
|f|_{\tau} \leq|f|_{\rho}^{c}|f|_{\sigma}^{1-c} \text {. }
$$


Proof. See [Kedlaya 2007, Lemma 3.1.6], [Amice 1975, Corollaire 4.2.8], or [Robba and Christol 1994, Corollaire 5.4.9].

Definition 2.4.5. For $I$ an aligned interval, a $\nabla$-module on $A_{L}(I)$ (relative to $K$ ) is a coherent locally free sheaf $\mathscr{E}$ on $A_{L}(I)$ equipped with an integrable $K$ linear connection $\nabla: \mathscr{E} \rightarrow \mathscr{E} \otimes \Omega_{A_{L}(I) / K}^{1}$. (Here $\Omega_{A_{L}(I) / K}^{1}$ denotes the sheaf of continuous differentials; it is freely generated over $O_{A_{L}(I)}$ by $d u_{1}, \ldots, d u_{n}, d t$.) The connection equips $\mathscr{E}$ with actions of the derivations $\partial_{i}=\frac{\partial}{\partial u_{i}}$ for $i=1, \ldots, n$ and $\partial_{n+1}=\frac{\partial}{\partial t}$; integrability of the connection is equivalent to commutativity between these actions.

Definition 2.4.6. For $I$ an aligned interval and $\rho \in I$, let $F_{\rho}$ be the completion of $L(t)$ for the $\rho$-Gauss norm, viewed as a differential field of order $n+1$. For $\mathscr{E}$ a nonzero $\nabla$-module on $A_{L}(I)$, let $J$ be a closed aligned neighborhood of $\rho$ in $I$, and put

$$
\mathscr{E}_{\rho}=\Gamma\left(A_{L}(J), \mathscr{E}\right) \otimes_{\Gamma\left(A_{L}(J), \mathscr{O}\right)} F_{\rho},
$$

viewed as a differential module over $F_{\rho}$; this construction does not depend on $J$. Define the radius multiset of $\mathscr{E}_{\rho}$, denoted $S(\mathscr{E}, \rho)$, as the multiset of reciprocals of the scale multiset of $\mathscr{E}_{\rho}$. Define the (toric) generic radius of convergence of $\mathscr{E}_{\rho}$, denoted $T(\mathscr{E}, \rho)$, as the smallest element of $S(\mathscr{E}, \rho)$, i.e., the reciprocal of the scale of $\mathscr{E}_{\rho}$.

Remark 2.4.7. As in [Kedlaya 2006b], the toric generic radius of convergence is normalized differently from the generic radius of convergence of [Christol and Dwork 1994], which would be multiplied by an extra factor of $\rho$. Our chief justification for this normalization is "because it works", in the sense of giving the expected answer for Example 3.5.10. We look forward to ongoing work of Baldassarri (compare Remark 2.2.4) for a more intrinsic justification.

Remark 2.4.8. To our knowledge, the consideration of $\nabla$-modules over a rigid analytic annulus, but taking into account derivations of the base field over a subfield, is novel to this paper. It may prove an interesting exercise to transcribe the arguments of [Kedlaya 2005a], such as local duality, as much as possible to this setting.

\subsection{The highest ramification break.}

Definition 2.5.1. Let $\mathscr{E}$ be a $\nabla$-module on $A_{L}(\epsilon, 1)$ for some $\epsilon \in(0,1)$. We say $\mathscr{E}$ is solvable at 1 if

$$
\lim _{\rho \rightarrow 1^{-}} T(\mathscr{E}, \rho)=1 .
$$

Hypothesis 2.5.2. For the rest of this subsection, let $\mathscr{E}$ be a $\nabla$-module on $A_{L}(\epsilon, 1)$ for some $\epsilon \in(0,1)$, which is solvable at 1 . 
Lemma 2.5.3. For each $i \in\{1, \ldots, n+1\}$, for $r \in(0,-\log \epsilon)$, put $\rho=e^{-r}$ and let $f_{i}(r)$ be the negative logarithm of the scale of $\partial_{i}$ on $\mathscr{E}_{\rho}$. Then $f_{i}$ is a concave function of $r$; in particular,

$$
\log T\left(\mathscr{E}, e^{-r}\right)=\min _{i}\left\{f_{i}(r)\right\}
$$

is a concave function of $r$. (This does not require solvability at 1.)

Proof. It suffices to check concavity on $-\log (J)$ for $J$ an arbitrary closed aligned subinterval of $(\epsilon, 1)$. Since $J$ is closed aligned, $A_{L}(J)$ is affinoid; by Kiehl's theorem (see for instance [Fresnel and van der Put 2004, Theorem 4.5.2]), $\Gamma\left(A_{L}(J), \mathscr{E}\right)$ is a finitely generated module over the ring $\Gamma\left(A_{L}(J), \mathcal{O}\right)$. Since that ring is a principal ideal domain [Lazard 1962, Proposition 4, Corollaire], $\Gamma\left(A_{L}(J), \mathscr{E}\right)$ is freely generated by some subset $\mathbf{e}_{1}, \ldots, \mathbf{e}_{m}$. Let $D_{i, l}$ be the matrix over $\Gamma\left(A_{L}(J), \mathcal{O}\right)$ via which $\partial_{i}^{l}$ acts on $\mathbf{e}_{1}, \ldots, \mathbf{e}_{m}$. For $\rho, \sigma \in J$ and $c \in[0,1]$, put $\tau=\rho^{c} \sigma^{1-c}$. By Lemma 2.4.4, we have

$$
\left|D_{i, l}\right|_{\tau} \leq\left|D_{i, l}\right|_{\rho}^{c}\left|D_{i, l}\right|_{\sigma}^{1-c}
$$

taking $l$-th roots of both sides and taking limits yields

$$
\limsup _{l \rightarrow \infty}\left|D_{i, l}\right|_{\tau}^{1 / l} \leq\left(\limsup _{l \rightarrow \infty}\left|D_{i, l}\right|_{\rho}^{1 / l}\right)^{c}\left(\limsup _{l \rightarrow \infty}\left|D_{i, l}\right|_{\sigma}^{1 / l}\right)^{1-c} .
$$

By (1.1.7.1), this yields the desired result. (Compare [Kedlaya 2006b, Proposition 4.2.6].)

Proposition 2.5.4. The function $f(r)=\log T\left(\mathscr{E}, e^{-r}\right)$ on $(0,-\log \epsilon)$ is piecewise linear, with slopes in $(1 /(\operatorname{rank} \mathscr{E}) !) \mathbb{Z}$. Moreover, $f$ is linear in a neighborhood of 0 .

Proof. Since $f$ is concave by Lemma 2.5.3, takes nonpositive values, and tends to 0 as $r \rightarrow 0^{+}$, it is everywhere nonincreasing. Hence for sufficiently large integers $h$, we can choose $\rho_{h} \in(\epsilon, 1)$ with $T\left(\mathscr{E}, \rho_{h}\right)=|p|^{1 /\left(p^{h-1}(p-1)\right)}$ and $\rho_{h}<\rho_{h+1}$. Put $r_{h}=-\log \rho_{h}$.

We now check piecewise linearity and the slope restriction on $\left(r_{h+1}, r_{h}\right)$; it suffices to check on $-\log (J)$ for $J$ an arbitrary closed aligned subinterval of $\left(\rho_{h}, \rho_{h+1}\right)$. Assume without loss of generality that $K$ contains a primitive $p$-th root of unity. Put $L_{0}=L$. For $l=1, \ldots, h$, let $L_{l}$ be the subfield of $L_{l-1}$ fixed under the action of $(\mathbb{Z} / p \mathbb{Z})^{n}$ given in Definition 2.3.1, but with

$$
u_{1}^{p^{l-1}}, \ldots, u_{n}^{p^{l-1}}
$$

playing the roles of $u_{1}, \ldots, u_{n}$. Since $T(\mathscr{E}, \rho)>|p|^{1 /\left(p^{h-1}(p-1)\right)}$ for $\rho \in J$, using Definition 2.3.1 (in the $u_{1}, \ldots, u_{n}$-directions) and [Kedlaya 2005a, Theorem 6.15] (in the $t$-direction), we can construct an $h$-fold Frobenius antecedent $\mathscr{E}_{h}$ for $\mathscr{E}$, which is defined on $A_{L_{h}}\left(J^{p^{h}}\right)$. 
Apply Lemma 1.1.5 to construct a cyclic vector for $\mathscr{E}_{h}$ over Frac $\Gamma\left(A_{L_{h}}\left(J^{p^{h}}\right), \mathcal{O}\right)$; by writing down the corresponding twisted polynomial $P(T)$ and applying Proposition 1.1.9, we see that for $\sigma \in J^{p^{h}}, T\left(\mathscr{E}_{h}, \sigma\right)$ is piecewise of the form $|g|_{\sigma}^{1 / j}$ for some $g \in \operatorname{Frac} \Gamma\left(A_{L_{h}}\left(J^{p^{h}}\right), \mathcal{O}\right)$ and $j \in\{1, \ldots, \operatorname{rank}(\mathscr{E})\}$. In particular, for $\sigma=\rho^{p^{h}}$, this expression is piecewise of the form $\left(|a| \rho^{i p^{h}}\right)^{1 / j}$ for some $a \in K^{*}, i \in \mathbb{Z}$, and $j \in\{1, \ldots, \operatorname{rank}(\mathscr{E})\}$. This proves that on $\left(r_{h+1}, r_{h}\right), f$ is piecewise linear with slopes in $(1 /(\operatorname{rank} \mathscr{E}) !) \mathbb{Z}$.

To check piecewise linearity in a neighborhood of $r_{h}$, note that as we approach $r_{h}$ from the right, the successive slopes of $f$ that we encounter are increasing but bounded above, and lie in a discrete subset of $\mathbb{R}$. Hence they stabilize, so $f$ is linear in a one-sided neighborhood of $r_{h}$. An analogous argument applies again when approaching $r_{h+1}$ from the left, so $f$ is piecewise linear on $\left[r_{h+1}, r_{h}\right]$; taking the union of these intervals, we deduce that $f$ is piecewise linear on $\left(0, r_{h}\right]$ for some $h$. An analogous argument applies yet again when approaching 0 from the right, yielding the desired result. (Compare [Christol and Mebkhout 2000, Théorème 4.21].)

Corollary 2.5.5. There exists $b \in \mathbb{Q}_{\geq 0}$ such that $T(\mathscr{E}, \rho)=\rho^{b}$ for all $\rho \in(\epsilon, 1)$.

Definition 2.5.6. We will refer to the number $b$ in Corollary 2.5 .5 as the (differential) highest ramification break of $\mathscr{E}$, denoted $b(\mathscr{E})$.

\subsection{Invariance.}

Definition 2.6.1. Define the Robba ring over $L$ as

$$
\mathscr{R}_{L}=\bigcup_{\epsilon \in(0,1)} \Gamma\left(A_{L}(\epsilon, 1), \mathcal{O}\right) .
$$

The elements of $\mathscr{R}_{L}$ can be represented as formal Laurent series $\sum_{i \in \mathbb{Z}} c_{i} t^{i}$ with $c_{i} \in L$; let $\mathscr{R}_{L}^{\text {int }}$ be the subring of series with $\left|c_{i}\right| \leq 1$ for all $i \in \mathbb{Z}$. The ring $\mathscr{R}_{L}^{\text {int }}$ is local, with maximal ideal consisting of series with $\left|c_{i}\right|<1$ for all $i \in \mathbb{Z}$, with residue field $\ell((t))$.

We first examine invariance under certain endomorphisms of $L$, following Definition 2.2.2.

Definition 2.6.2. Choose $u_{1}^{\prime}, \ldots, u_{n}^{\prime}, t^{\prime} \in \mathscr{R}_{L}^{\text {int }}$ such that under the projection $\mathscr{R}_{L}^{\mathrm{int}} \rightarrow$ $\ell((t)), u_{1}^{\prime}-u_{1}, \ldots, u_{n}^{\prime}-u_{n}$ map into $t \ell \llbracket t \rrbracket$ and $t^{\prime}-t$ maps into $t^{2} \ell \llbracket t \rrbracket$. Then for some $\epsilon \in(0,1)$, the Taylor series

$$
\sum_{I \in \mathbb{N}_{0}^{n+1}} \frac{\left(u_{1}^{\prime}-u_{1}\right)^{i_{1}} \cdots\left(u_{n}^{\prime}-u_{n}\right)^{i_{n}}\left(t^{\prime}-t\right)^{i_{n+1}}}{I !} \partial^{I}(f)
$$


converges for $f \in \Gamma\left(A_{L}(I), \mathcal{O}\right)$ for any closed aligned subinterval $I$ of $(\epsilon, 1)$, so we can use it to define a map $g: \Gamma\left(A_{L}(I), \mathcal{O}\right) \rightarrow \Gamma\left(A_{L}(I), \mathcal{O}\right)$ such that $g^{*}\left(u_{i}\right)=u_{i}^{\prime}$, $g^{*}(t)=t^{\prime}$.

Proposition 2.6.3. Let $g$ be a map as in Definition 2.6.2. For any $\nabla$-module $\mathscr{E}$ on $A_{L}(\epsilon, 1)$ which is solvable at 1 , we have $T(\mathscr{E}, \rho)=T\left(g^{* \mathscr{E}}, \rho\right)$ for all $\rho \in(\epsilon, 1)$ sufficiently close to 1 . In particular, $g^{* \mathscr{E}}$ is also solvable at 1 , and $\mathscr{E}$ and $g^{* \mathscr{E}}$ have the same highest break.

Proof. By the choice of $u_{1}^{\prime}, \ldots, u_{n}^{\prime}, t^{\prime}$, for $\rho \in(0,1)$ sufficiently close to 1 ,

$$
\left|u_{i}^{\prime}-u_{i}\right|_{\rho}<1 \quad(i=1, \ldots, n), \quad\left|t^{\prime}-t\right|_{\rho}<\rho .
$$

We will prove the claim for such $\rho$.

By continuity of $T(\mathscr{E}, \rho)$ (implied by Lemma 2.5.3), it suffices to check for $\rho \in \Gamma^{*}$. There is no loss of generality in enlarging $K$, so we may in fact assume that there exists $\lambda \in K$ with $|\lambda|=\rho$. In this case, we may put ourselves in the situation of Remark 2.2 .4 by considering $\mathscr{E}_{\rho}$ to be a differential module over the completion of $L(t / \rho)$ for the 1-Gauss norm, comparing the $p$-bases $u_{1}, \ldots, u_{n}, t / \lambda$ and $u_{1}^{\prime}, \ldots, u_{n}^{\prime}, t^{\prime} / \lambda$. This yields the claim.

Proposition 2.6.4. Let $g: A_{L}(I) \rightarrow A_{L}(I)$ be the map fixing $L$ and pulling $t$ back to $t^{p^{N}}$ for some positive integer $N$. Then for any $\nabla$-module $\mathscr{E}$ on $A_{L}(\epsilon, 1)$, we have $S(\mathscr{E}, \rho) \leq S\left(g^{* \mathscr{E}}, \rho^{1 / p^{N}}\right)$ for all $\rho \in(\epsilon, 1)$; moreover, if $n=0$, then $S(\mathscr{E}, \rho) \leq S\left(g^{* \mathscr{E}}, \rho^{1 / p^{N}}\right)^{p^{N}}$.

Proof. If we compare the scale multisets of $\partial_{i}$ on $\mathscr{E}_{\rho}$ and on $\left(g^{* \mathscr{E})}\right)_{\rho^{1 / p^{N}}}$, then we get identical results for $i=1, \ldots, n$. For $i=n+1$, the scale multiset on $\mathscr{E}_{\rho}$ is at least the $p^{N}$-th power of the scale multiset on $\left(g^{* \mathscr{C}}\right) \rho_{\rho^{1 / p^{N}}}$, as in the proof of Proposition 2.3.2. This yields the claim.

Proposition 2.6.5. Let $g: A_{L}(I) \rightarrow A_{L}(I)$ be the map fixing $L$ and pulling $t$ back to $t^{N}$ for some positive integer $N$ coprime to $p$. Then for any $\nabla$-module $\mathscr{E}$ on $A_{L}(\epsilon, 1)$, we have $S(\mathscr{E}, \rho)=S\left(g^{* \mathscr{E}}, \rho^{1 / N}\right)$ for all $\rho \in(\epsilon, 1)$.

Proof. If we compare the scale multisets of $\partial_{i}$ on $\mathscr{E}_{\rho}$ and on $\left(g^{* \mathscr{E}}\right)_{\rho^{1 / N}}$, then we get identical results for $i=1, \ldots, n$. For $i=n+1$, we again get identical results by virtue of [Kedlaya 2005a, Lemma 5.11].

We next examine what happens when we change the $p$-basis.

Proposition 2.6.6. Choose $u_{1}^{\prime}, \ldots, u_{n}^{\prime} \in \mathscr{R}_{L}^{\mathrm{int}}$ such that under the projection $\mathscr{R}_{L}^{\mathrm{int}} \rightarrow$ $\ell((t)), u_{1}^{\prime}, \ldots, u_{n}^{\prime}$ map to elements of $\ell \llbracket t \rrbracket$ lifting a p-basis of $\ell$ over $k$. Let $\partial_{1}^{\prime}, \ldots, \partial_{n}^{\prime}$ be the derivations dual to the basis $d u_{1}^{\prime}, \ldots, d u_{n}^{\prime}$ of $\Omega_{L / K}^{1}$. Let $\mathscr{E}$ be $a \nabla$-module on $A_{L}(\epsilon, 1)$ for some $\epsilon \in(0,1)$, which is solvable at 1 . Then for $\rho \in(0,1)$ sufficiently close to 1 , the scale of $\mathscr{E}_{\rho}$ for $\partial_{1}, \ldots, \partial_{n}, \partial_{n+1}$ is the same as for $\partial_{1}^{\prime}, \ldots, \partial_{n}^{\prime}, \partial_{n+1}$; in particular, the highest break is the same in both cases. 
Proof. If $u_{1}^{\prime}, \ldots, u_{n}^{\prime} \in L$, then we can invoke Remark 2.2.4 to obtain the claim. In general, we may first make a transformation as in the previous sentence, to match up the reductions modulo $t \ell \llbracket t \rrbracket$, then invoke Proposition 2.6.3.

2.7. The break decomposition. Retain Hypothesis 2.5 .2 throughout this subsection.

Definition 2.7.1. We say that $\mathscr{E}$ has a uniform break if for all $\rho \in(0,1)$ sufficiently close to $1, S(\mathscr{E}, \rho)$ consists of a single element with multiplicity $\operatorname{rank}(\mathscr{E})$. We write "E् has uniform break $b$ " as shorthand for " $\mathscr{E}$ has a uniform break and its highest ramification break is $b$ ".

Theorem 2.7.2. For some $\eta \in(0,1)$, there exists a decomposition of $\nabla$-modules (necessarily unique) $\mathscr{\mathscr { E }}=\oplus_{b \in \mathbb{Q}_{\geq 0}} \mathscr{E}_{b}$ over $A_{L}(\eta, 1)$ such that each $\mathscr{E}_{b}$ has uniform break $b$.

We will prove Theorem 2.7.2 later in this subsection. To begin with, we recall that the case $L=K$ is essentially a theorem of Christol-Mebkhout [2001, Corollaire 2.4-1], from which we will bootstrap to the general case.

Lemma 2.7.3. Theorem 2.7.2 holds in case $L=K$.

Proof. This is the conclusion of [Christol and Mebkhout 2001, Corollaire 2.4-1], at least in case $K$ is spherically complete. However, it extends to the general case as follows.

By a straightforward application of Zorn's lemma, we may embed $K$ into a spherically complete field $K^{\prime}$. Apply [Christol and Mebkhout 2001, Corollaire 2.41] to obtain a break decomposition over $A_{K^{\prime}}(\eta, 1)$ for some $\eta \in(0,1)$; let $\mathbf{v} \in$ $\Gamma\left(A_{K^{\prime}}(\eta, 1), \mathscr{E} \vee \otimes \mathscr{E}\right)$ be the projector onto the highest break component.

Now set notation as in the proof of Proposition 2.5.4. The set of $\rho \in\left(\rho_{h}, \rho_{h+1}\right)$ for which at least one coefficient $P(T)$ fails to be a unit in $A_{L_{h}}\left[\rho^{p^{h}}, \rho^{p^{h}}\right]$ is discrete, so we may choose $\rho \in\left(\rho_{h}, \rho_{h+1}\right)$ not of that form. Then Proposition 1.1.10 gives a factorization of $P(T)$ over $A_{L_{h}}\left[\rho^{p^{h}}, \rho^{p^{h}}\right]$ (and likewise in the opposite ring); we thus obtain an element $\mathbf{v}^{\prime}$ of $\Gamma\left(A_{K}[\rho, \rho], \mathscr{E}^{\vee} \otimes \mathscr{E}\right)$ which agrees with $\mathbf{v}$ over $A_{K^{\prime}}[\rho, \rho]$.

For any closed aligned subinterval $J$ of $(\eta, 1)$ containing $\rho$, we have

$$
\Gamma\left(A_{K}[\rho, \rho], \mathcal{O}\right) \cap \Gamma\left(A_{K^{\prime}}(J), \mathcal{O}\right)=\Gamma\left(A_{K}(J), \mathcal{O}\right)
$$

inside $\Gamma\left(A_{K^{\prime}}[\rho, \rho], \mathcal{O}\right)$. Since $\mathscr{E}^{\vee} \otimes \mathscr{E}$ is free over $A_{K}(J)$ (as in the proof of Lemma 2.5.3), this implies that

$$
\Gamma\left(A_{K}[\rho, \rho], \mathscr{E}^{\vee} \otimes \mathscr{E}\right) \cap \Gamma\left(A_{K^{\prime}}(J), \mathscr{E}^{\vee} \otimes \mathscr{E}\right)=\Gamma\left(A_{K}(J), \mathscr{E}^{\vee} \otimes \mathscr{E}\right),
$$


and so $\mathbf{v} \in \Gamma\left(A_{K}(J), \mathscr{E}^{\vee} \otimes \mathscr{E}\right)$. Running this argument over all possible $J$, we obtain $\mathbf{v} \in \Gamma\left(A_{K}(\eta, 1), \mathscr{E}^{\vee} \otimes \mathscr{E}\right)$, so $\mathscr{E}$ admits a break decomposition over $A_{K}(\eta, 1)$ as desired.

We exploit Lemma 2.7.3 via the following construction.

Definition 2.7.4. Define the relativization $\mathscr{F}$ of $\mathscr{E}$ as the $\nabla$-module $\mathscr{E}$ itself, but viewed relative to $L$ instead of $K$. That is, retain only the action of $\partial_{n+1}$. (The term "generic fibre" was used in an earlier version of this paper, but we decided to reserve that name for a different concept to appear in a subsequent paper.)

However, we are forced to make a crucial distinction.

Lemma 2.7.5. For $i \in\{1, \ldots, n+1\}$, there exists $\eta \in(0,1)$ such that one of the following two statements is true.

- For all $\rho \in(\eta, 1), \partial_{i}$ is dominant for $\mathscr{E}_{\rho}$.

- For all $\rho \in(\eta, 1), \partial_{i}$ is not dominant for $\mathscr{E}_{\rho}$.

Proof. Let $b$ denote the highest break of $\mathscr{E}$. Choose $\eta \in(0,1)$ such that $T(\mathscr{E}, \rho)=\rho^{b}$ for all $\rho \in(\eta, 1)$. Put

$$
f_{i}(\rho)=\frac{\left|\partial_{i}\right|_{F_{\rho}, \mathrm{sp}}}{\left|\partial_{i}\right|_{\mathscr{E}_{\rho}, \mathrm{sp}}}
$$

then Lemma 2.5.3 shows that $f_{i}$ is log-concave. Consequently, if $f_{i}(\rho)=T(\mathscr{E}, \rho)$ for two different values of $\rho$, then the same is true for all intermediate values. This proves the claim: if the second statement does not hold, then there exist $\rho \in(0,1)$ arbitrarily close to 1 such that $f_{i}(\rho)=T(\mathscr{E}, \rho)$, in which case the first statement holds with $\eta$ equal to any such $\rho$.

Definition 2.7.6. For $i \in\{1, \ldots, n+1\}$, we say that $\partial_{i}$ is eventually dominant for $\mathscr{E}$ if the first alternative in Lemma 2.7 .5 holds, i.e., if there exists $\eta \in(0,1)$ such that for all $\rho \in(\eta, 1), \partial_{i}$ is dominant for $\mathscr{E}_{\rho}$.

Remark 2.7.7. Note that if $\partial_{n+1}$ is eventually dominant for $\mathscr{E}$, then the highest break term in the decomposition of $\mathscr{F}$ (which is respected by $\partial_{1}, \ldots, \partial_{n}$ because it is unique) already has a uniform break. Our strategy in case $\partial_{n+1}$ is not eventually dominant for $\mathscr{E}$ is to perform an operation which one might call rotation to recover that more favorable situation: namely, we use a concrete Taylor isomorphism to change the embedding of $K$ into $L$.

In order to perform the rotation suggested in Remark 2.7.7, we need two particular instances of Definition 2.6.2.

Lemma 2.7.8. For $N$ a nonnegative integer, let $f_{N}: A_{L}(0,1) \rightarrow A_{L}(0,1)$ be the map fixing $L$ and pulling back $t$ to $t^{p^{N}}$. Then for $\rho \in(\epsilon, 1)$, we have the inequality $S\left(f_{N}^{* \mathscr{E}}, \rho^{1 / p^{N}}\right) \geq S(\mathscr{E}, \rho)$. Moreover, if $\partial_{i}$ is dominant for $\mathscr{E}_{\rho}$ for some $i \neq n+1$, then $T\left(f_{N}^{* \mathscr{E}}, \rho^{1 / p^{N}}\right)=T(\mathscr{E}, \rho)$. 
Proof. The first assertion follows from Proposition 2.6.4. The second follows because if $\partial_{i}$ is dominant for $\mathscr{E}_{\rho}$ and $i \neq n+1$, then $T\left(f_{N}^{* \mathscr{E}}, \rho^{1 / p^{N}}\right)$ and $T(\mathscr{E}, \rho)$ can be computed using the same formula.

Lemma 2.7.9. Suppose $i \in\{1, \ldots, n\}$ is such that $\partial_{i}$ is eventually dominant for $\mathscr{E}$. Let $g_{i}$ be the map given by Definition 2.6.2 with

$$
u_{i}^{\prime}=u_{i}+t, \quad u_{j}^{\prime}=u_{j} \quad(j \neq i), \quad t^{\prime}=t .
$$

Put $\mathscr{E}^{\prime}=g_{i}^{* \mathscr{E}}$, and let $\mathscr{F}^{\prime}$ be the relativization of $\mathscr{E}^{\prime}$. Let $b, b_{\text {rel }}$ be the highest breaks of $\mathscr{E}, \mathscr{F}$. If $b>b_{\text {rel }}+1$, then:

- the highest break of $\mathscr{F}^{\prime}$ is $b-1$;

- for $\rho \in(0,1)$ sufficiently close to 1 , the multiplicity of $\rho^{b-1}$ in $S\left(\mathscr{F}^{\prime}, \rho\right)$ is the same as that of $\rho^{b}$ in $S(\mathscr{E}, \rho)$.

Proof. The action of $\partial_{n+1}$ on $g_{i}^{* \mathscr{E}}$ is the pullback of the action of $\partial_{n+1}+\partial_{i}$ on $\mathscr{E}$, so the highest break of $\mathscr{F}^{\prime}$ is the value of $b^{\prime}$ satisfying

$$
\left|\partial_{n+1}+\partial_{i}\right|_{\mathscr{E}_{\rho}, \mathrm{sp}}=\rho^{-b^{\prime}-1}
$$

for $\rho \in(0,1)$ sufficiently close to 1 . For such $\rho$, the spectral norms of $\partial_{i}, \partial_{n+1}$ on $\mathscr{E}_{\rho}$ are $\rho^{-b}, \rho^{-b_{\text {rel }}-1}$, respectively. From this the claims are evident.

Lemma 2.7.10. Pick $i \in\{1, \ldots, n+1\}$ such that $\partial_{i}$ is eventually dominant for $\mathscr{E}$. Then at least one of the following statements is true.

- For $\rho \in(0,1)$ sufficiently close to 1 , the scale multiset of $\partial_{i}$ on $\mathscr{E}_{\rho}$ consists of a single element.

- There exists $\eta \in(0,1)$ such that $\mathscr{E}$ is decomposable on $A_{L}(\eta, 1)$.

Proof. If $i=n+1$, then the claim follows by Remark 2.7.7, so we assume $i \leq n$. Let $b$ and $b_{\text {rel }}$ be the highest breaks of $\mathscr{E}$ and $\mathscr{F}$, respectively. Assume that the first alternative does not hold; this forces $b>0$.

Suppose to begin with that $b>b_{\text {rel }}+1$. Put $\mathscr{C}^{\prime}=g_{i}^{* \mathscr{E}}$ as in Lemma 2.7.9, and let $\mathscr{F}^{\prime}$ be the relativization of $\mathscr{E}^{\prime}$. Then $\mathscr{F}^{\prime}$ does not have a uniform break, so by Lemma 2.7.3, it splits off a component of uniform break $b-1$. We conclude that $\mathscr{E}^{\prime}$ is decomposable on some $A_{L}(\eta, 1)$, as then is $\mathscr{E}$, as desired.

In the general case, we can always pick $N$ such that $b p^{N}>b_{\text {rel }}+1$. By Lemma 2.7.8, $f_{N}^{* \mathscr{E}}$ has highest break $b p^{N}$, and the first alternative of this lemma also does not hold for $f_{N}^{* \mathscr{E}}$. Moreover, by Proposition 2.3.2, the relativization of $f_{N}^{* \mathscr{E}}$ has highest break $b_{\text {rel }}$. We may thus apply the previous paragraph to split off a component of $f_{N}^{* \mathscr{E}}$ of highest break; since the splitting is unique, it descends down the Galois group of the cover $f_{N}$ (after adjoining $p^{N}$-th roots of unity), so $\mathscr{E}$ is itself decomposable on some $A_{L}(\eta, 1)$, as desired. 
Proof of Theorem 2.7.2. It suffices to show that if $\mathscr{E}$ is indecomposable over $A_{L}(\eta, 1)$ for any $\eta \in(0,1)$ sufficiently close to 1 , then $\mathscr{E}$ has a uniform break. This follows from Remark 2.7.7 if $\partial_{n+1}$ is eventually dominant for $\mathscr{E}$, and from Lemma 2.7.10 otherwise.

It will be useful later to have a more uniform version of the rotation construction used in Section 2.7, which comes at the expense of enlarging the field $L$. (This generic rotation is inspired by the operation of generic residual perfection in [Borger 2004].) The resulting construction will be used to study the graded pieces of the ramification filtration.

Proposition 2.7.11. Let $b$ be the highest break of $\mathscr{E}$, and suppose $b>1$. Let $L^{\prime}$ be the completion of $L\left(v_{1}, \ldots, v_{n}\right)$ for the $(1, \ldots, 1)$-Gauss norm, viewed as a differential field of order $2 n$ over $K$. Let $\mathscr{E}^{\prime}$ be the pullback of $\mathscr{E}$ along the map $f: A_{L^{\prime}}[0,1) \rightarrow A_{L}[0,1)$ given by

$$
f^{*}\left(u_{i}\right)=u_{i}^{p}+v_{i} t^{p-1} \quad(i=1, \ldots, n), \quad f^{*}(t)=\frac{t^{p}}{1-t^{p-1}} .
$$

Then $\mathscr{C}^{\prime}$ ' has highest break $p b-p+1$. In addition, among the differentials

$$
\frac{\partial}{\partial u_{1}}, \ldots, \frac{\partial}{\partial u_{n}}, \frac{\partial}{\partial v_{1}}, \ldots, \frac{\partial}{\partial v_{n}}, \frac{\partial}{\partial t},
$$

$\frac{\partial}{\partial t}$ (at least) is eventually dominant for $\mathscr{E}^{\prime}$.

Proof. We first treat the case $n=0$. In this case, $g^{*}\left(t^{-1}\right)=t^{-p}-t^{-1}$, so this is an instance of [Kedlaya 2005a, Lemma 5.13].

In the general case, writing $\partial_{1}^{\prime}, \ldots, \partial_{n+1}^{\prime}$ for the actions of $\partial_{1}, \ldots, \partial_{n+1}$ before the pullback, we have

$$
\begin{aligned}
\frac{\partial}{\partial u_{i}} & =p u_{i}^{p-1} \partial_{i}^{\prime}, \\
\frac{\partial}{\partial v_{i}} & =t^{p-1} \partial_{i}^{\prime}, \\
\frac{\partial}{\partial t} & =\frac{d}{d t}\left(\frac{t^{p}}{1-t^{p-1}}\right) \partial_{n+1}^{\prime}+\sum_{i=1}^{n}(p-1) v_{i} t^{p-2} \partial_{i}^{\prime} .
\end{aligned}
$$

We compute the scale of $\partial / \partial t$ by inspecting each term separately: the contribution from $\partial_{n+1}^{\prime}$ can be treated as above, and the contribution from $\partial_{i}^{\prime}$ can be treated directly after invoking Proposition 2.6.5. This implies that the highest break of $\mathscr{E}^{\prime}$ is at least $p b-p+1$, with equality if and only if $\partial / \partial t$ is eventually dominant.

We compute the scale of $\partial / \partial u_{i}$ as if $u_{i}$ had pulled back to $u_{i}^{p}$ and $t$ to $t^{p}$ (i.e., as for a Frobenius antecedent). In particular, if $\partial / \partial u_{i}$ were eventually dominant for 
$\mathscr{E}^{\prime}$, then the highest break of $\mathscr{E}^{\prime}$ would be at most $b<p b-p+1$, contradiction. Hence $\partial / \partial u_{i}$ is not eventually dominant.

We read off the scale of $\partial / \partial v_{i}$ directly: it is eventually dominant if and only if $\partial_{i}^{\prime}$ is, and in any case it cannot mask $\partial / \partial t$. This proves the desired results.

Remark 2.7.12. The calculations in this subsection may become more transparent when checked against the examples produced by Artin-Schreier covers in positive characteristic, as in Example 3.5.10. Indeed, many of these calculations were conceived with those examples firmly in mind.

2.8. The differential Swan conductor. Throughout this subsection, retain Hypothesis 2.5.2.

Definition 2.8.1. By Theorem 2.7.2, there exists a multiset $\left\{b_{1}, \ldots, b_{d}\right\}$ such that for all $\rho \in(0,1)$ sufficiently close to $1, S(\mathscr{E}, \rho)=\left\{\rho^{b_{1}}, \ldots, \rho^{b_{d}}\right\}$. We call this multiset the break multiset of $\mathscr{E}$, denoted $b(\mathscr{E})$. Define the (differential) Swan conductor of $\mathscr{E}$, denoted Swan $(\mathscr{E})$, as $b_{1}+\cdots+b_{d}$.

Theorem 2.8.2. The differential Swan conductor of $\mathscr{E}$ is a nonnegative integer.

Proof. It suffices to check this in case $\mathscr{E}$ is indecomposable over $A_{L}(\eta, 1)$ for any $\eta \in(0,1)$ sufficiently close to 1 . Choose $i \in\{1, \ldots, n+1\}$ such that $\partial_{i}$ is eventually dominant for $\mathscr{E}$. By Lemma 2.7.10, for $\rho \in(\epsilon, 1)$ sufficiently close to 1 , the scale multiset of $\mathscr{E}_{\rho}$ with respect to $\partial_{i}$ consists of a single element. That means in the calculation of the Newton polygon in Proposition 2.5.4, the Newton polygon must have only one slope, and so the integer $j$ can be taken to be $\operatorname{rank}(\mathscr{E})$. Consequently, the slopes of the function $f(r)=\log T\left(\mathscr{E}, e^{-r}\right)$ are always multiples of $1 / \operatorname{rank}(\mathscr{E})$, as then is the highest break of $\mathscr{E}$. This proves the desired result.

Remark 2.8.3. Proposition 2.6 .5 implies that pulling $\mathscr{E}$ along the map $t \mapsto t^{N}$, for $N$ a positive integer coprime to $p$, has the effect of multiplying $\operatorname{Swan}(\mathscr{E})$ by $N$. For Galois representations, this will imply that the Swan conductor commutes appropriately with tamely ramified base changes (Theorem 3.5.9).

Remark 2.8.4. In case $L=K$, one can interpret the integrality of $\operatorname{Swan}(\mathscr{E})$ by equating it to a certain local index [Christol and Mebkhout 2001, Théorème 2.31]. It would be interesting to give a cohomological interpretation of our more general construction, perhaps by relating it to an appropriate Euler characteristic.

Remark 2.8.5. Liang Xiao points out that one can also prove Theorem 2.8.2 by reduction to the case of perfect residue field, for which one may invoke Remark 2.8.4. The argument is as follows. By Lemma 2.7.10, we may assume that $\mathscr{E}$ and its relativization have respective uniform breaks $b, b_{\text {rel }}$. The perfect residue field case implies that $b_{\text {rel }} \operatorname{rank}(\mathscr{E})$ is an integer. If $b \neq b_{\text {rel }}$, we can choose positive integers $m_{1}, m_{2}$ coprime to each other and to $p$ such that $m_{i}\left(b-b_{\text {rel }}\right)>1$ for $i=1,2$. If we 
pull back along $t \mapsto t^{m_{i}}$ and then apply the rotation in Lemma 2.7.9, the highest break of the relativization becomes $m_{i} b-1$, so $\left(m_{i} b-1\right) \operatorname{rank}(\mathscr{E})$ is an integer for $i=1,2$. This implies that $b \operatorname{rank}(\mathscr{E}) \in \mathbb{Z}$.

\section{Differential conductors for Galois representations}

In this section, we explain how to define differential Swan conductors for certain $p$-adic Galois representations of complete discretely valued fields of equal characteristic $p>0$ (including the discrete representations). This uses a setup for turning representations into differential modules due to [Tsuzuki 1998]. For comments on the mixed characteristic case, see Section 3.7.

\subsection{Preliminaries: Cohen rings.}

Definition 3.1.1. Let $k$ be a field of characteristic $p>0$. A Cohen ring for $k$ is a complete discrete valuation ring $C_{k}$ with maximal ideal generated by $p$, equipped with an isomorphism of its residue field with $k$.

It can be shown that Cohen rings exist and are unique up to noncanonical isomorphism; see [Bourbaki 1983]. One can do better by carrying some extra data.

Definition 3.1.2. Define a based field of characteristic $p>0$ to be a field $k$ equipped with a distinguished $p$-basis $B_{k}$. We view based fields as forming a category whose morphisms from $\left(k, B_{k}\right)$ to $\left(k^{\prime}, B_{k}^{\prime}\right)$ are morphisms $k \rightarrow k^{\prime}$ of fields carrying $B_{k}$ into $B_{k}^{\prime}$.

Definition 3.1.3. For $\left(k, B_{k}\right)$ a based field, a based Cohen ring for $\left(k, B_{k}\right)$ is a pair $(C, B)$, where $C$ is a Cohen ring for $k$ and $B$ is a subset of $C$ which lifts $B_{k}$.

Proposition 3.1.4. There is a functor from based fields to based Cohen rings which is a quasi-inverse of the residue field functor. In particular, any map between based fields lifts uniquely to given based Cohen rings.

Proof. This is implicit in Cohen's original paper [Cohen 1946]; an explicit proof is given in [Whitney 2002, Theorem 2.1]. Here is a sketch of another proof. Let $W_{n}$ be the ring of $p$-typical Witt vectors of length $n$ over $k$, let $W$ be the inverse limit of the $W_{n}$, let $F$ be the Frobenius on $W$, and let [.] denote the Teichmüller map. Put $B=\left\{[\bar{b}]: \bar{b} \in B_{k}\right\}$. Let $C_{n}$ be the image of $F^{n}(W)[B]$ in $W_{n}$. Then the projection $W_{n+1} \rightarrow W_{n}$ induces a surjection $C_{n+1} \rightarrow C_{n}$. Let $C$ be the inverse limit of the $C_{n}$; one then verifies that $(C, B)$ is a based Cohen ring for $\left(k, B_{k}\right)$, and functoriality of the construction follows from functoriality of the Witt ring.

Remark 3.1.5. In fact, [Whitney 2002, Theorem 2.1] asserts something slightly stronger: if $(C, B)$ is a based Cohen ring of $\left(k, B_{k}\right), R$ is any complete local ring with residue field $k$, and $B_{R}$ is a lift of $B_{k}$ to $R$, then there is a unique ring homomorphism $C \rightarrow R$ inducing the identity on $k$ and carrying $B$ to $B_{R}$. 


\subsection{Galois representations and $(\phi, \nabla)$-modules.}

Hypothesis 3.2.1. For the remainder of this section, let $R$ be a complete discrete valuation ring of equal characteristic $p>0$, with fraction field $E$ and residue field $k$. Let $k_{0}=\bigcap_{n \geq 0} k^{p^{n}}$ be the maximal perfect subfield of $k$; note that $k_{0}$ embeds canonically into $R$ (whereas if $k \neq k_{0}$, then $k$ embeds but not canonically).

Convention 3.2.2. Put $G_{E}=\operatorname{Gal}\left(E^{\mathrm{sep}} / E\right)$. Let $\mathcal{O}$ be the integral closure of $\mathbb{Z}_{p}$ in a finite extension of $\mathbb{Q}_{p}$, whose residue field $\mathbb{F}_{q}$ is contained in $k$. Throughout this section, a "representation" will be a continuous representation $\rho: G_{E} \rightarrow \operatorname{GL}(V)$, where $V=V(\rho)$ is a finite free 0 -module. (One can also consider representations on finite dimensional Frac(O)-vector spaces, by choosing lattices; for brevity, we stick to statements for integral representations, except for Remark 3.5.11.)

Definition 3.2.3. Fix a based Cohen ring $\left(C_{E}, B\right)$ with residue field $E$; note that $C_{E}$ is canonically a $W\left(\mathbb{F}_{q}\right)$-algebra. Put

$$
\Gamma=C_{E} \otimes_{W\left(\mathbb{F}_{q}\right)} \bigcirc .
$$

Let $\Omega_{\Gamma / \mathcal{O}}^{1}$ be the completed (for the $p$-adic topology) direct sum of $\Gamma d b$ over all $b \in B$, i.e., the inverse limit over $n$ of $\oplus_{b \in B}\left(\Gamma / p^{n} \Gamma\right) d b$; then there is a canonical derivation $d: \Gamma \rightarrow \Omega_{\Gamma / 0}^{1}$. Note that all of this data stays canonically independent of the choice of $B$ as long as $C_{E}$ remains fixed.

Definition 3.2.4. A $\nabla$-module over $\Gamma$ is a finite free $\Gamma$-module $M$ equipped with an integrable connection $\nabla: M \rightarrow M \otimes_{\Gamma} \Omega_{\Gamma / \mathcal{O}}^{1}$; integrability means that the composition of $\nabla$ with the map $M \otimes \Omega_{\Gamma / \mathcal{O}}^{1} \rightarrow M \otimes \wedge_{\Gamma}^{2} \Omega_{\Gamma / \mathcal{O}}^{1}$ induced by $\nabla$ is the zero map.

Definition 3.2.5. A Frobenius lift on $\Gamma$ is an endomorphism $\phi: \Gamma \rightarrow \Gamma$ fixing 0 and lifting the $q$-power Frobenius map on $E$. For instance, there is a unique such $\phi$ carrying $b$ to $b^{q}$ for each $b \in B$ (induced by the Frobenius action on the construction given in Proposition 3.1.4); we call this $\phi$ the standard Frobenius lift with respect to $B$. A $\phi$-module (resp. $(\phi, \nabla)$-module) over $\Gamma$ is a finite free module (resp. $\nabla$ module) $M$ over $\Gamma$ equipped with an isomorphism $F: \phi^{*} M \cong M$ of modules (resp. of $\nabla$-modules); we interpret $F$ as a semilinear action of $\phi$ on $M$.

Definition 3.2.6. For any representation $\rho$, put

$$
D(\rho)=\left(V(\rho) \otimes_{\mathcal{O}} \widehat{\Gamma^{\text {unr }}}\right)^{G_{E}} .
$$

By Hilbert's Theorem 90, the natural map

$$
D(\rho) \otimes_{\Gamma} \widehat{\Gamma^{\text {unr }}} \rightarrow V(\rho) \otimes_{\mathcal{C}} \widehat{\Gamma \text { unr }}
$$


is a bijection; in particular, $D(\rho)$ is a free $\Gamma$-module and $\operatorname{rank}_{\Gamma}(D(\rho))=\operatorname{rank}_{\mathscr{O}}(V)$. If we equip $\Gamma^{\mathrm{unr}}$ and its completion with actions of the derivation $d$ and any Frobenius lift $\phi$ (acting trivially on $V(\rho)$ ), we obtain by restriction a Frobenius action and connection on $D(\rho)$, turning it into a $(\phi, \nabla)$-module.

Proposition 3.2.7. For any Frobenius lift $\phi$ on $\Gamma$, the functor $D$ from representations to $\phi$-modules over $\Gamma$ is an equivalence of categories.

Proof. Given a $\phi$-module $M$ over $\Gamma$, put

$$
V(M)=\left(M \otimes_{\Gamma} \widehat{\Gamma^{\mathrm{unr}}}\right)^{\phi=1} .
$$

As in [Fontaine 1990, A1.2.6] or [Tsuzuki 1998, Theorem 4.1.3], one checks that $V$ is a quasi-inverse to $D$.

Proposition 3.2.8. For any Frobenius lift $\phi$ on $\Gamma$, any $\phi$-module over $\Gamma$ admits a unique structure of $(\phi, \nabla)$-module. Consequently, the functor $D$ from representations to $(\phi, \nabla)$-modules over $\Gamma$ is an equivalence of categories.

Proof. Existence of such a structure follows from Proposition 3.2.7, so we focus on uniqueness. Let $M$ be a $(\phi, \nabla)$-module over $\Gamma$. Let $\partial / \partial b$ be the derivations dual to the $d b$ for $b \in B$. Let $\mathbf{e}_{1}, \ldots, \mathbf{e}_{m}$ be a basis of $M$, and let $\Phi$ and $N_{b}$ be the matrices via which $\phi$ and $\partial / \partial b$ act on this basis. Then the fact that the $\phi$-action on $M$ respects the $\nabla$-module structure implies that

$$
N \Phi+\frac{\partial \Phi}{\partial b}=\frac{\partial \phi(b)}{\partial b} \Phi N .
$$

Let $\pi$ be a uniformizer of $\mathcal{O}$; note that $\partial \phi(b) / \phi b \equiv 0(\bmod \pi)$ because $\phi(b) \equiv b^{q}$ $(\bmod \pi)$. Consequently, for fixed $\Phi$, if $N_{b}$ is uniquely determined modulo $\pi^{m}$, then the right side of (3.2.8.1) is determined modulo $\pi^{m+1}$, as then is $N_{b} \Phi$. Since $\Phi$ is invertible, $N_{b}$ is also determined modulo $\pi^{m+1}$. By induction, $N_{b}$ is uniquely determined by $\Phi$ for each $b$, as desired.

3.3. Representations with finite local monodromy. We now distinguish the class of representations for which we define differential Swan conductors.

Definition 3.3.1. Let $I_{E}=\operatorname{Gal}\left(E^{\mathrm{sep}} / E^{\mathrm{unr}}\right)$ be the inertia subgroup of $G_{E}$. We say a representation $\rho$ has finite local monodromy if the image of $I_{E}$ under $\rho$ is finite.

For representations with finite local monodromy, we can refine the construction of the $(\phi, \nabla)$-module associated to $\rho$.

Hypothesis 3.3.2. For the remainder of this subsection, assume that $k$ admits a finite $p$-basis. Assume also that the based Cohen ring $\left(C_{E}, B\right)$ has been chosen with $B=B_{0} \cup\{t\}$, where $t$ lifts a uniformizer of $E$, and $B_{0}$ lifts elements of $R$ which in turn lift a $p$-basis of $k$. 
Definition 3.3.3. By the proof of the Cohen structure theorem, or by Remark 3.1.5, there is a unique embedding of $k$ into $R$ whose image contains the image of $B_{0}$ under reduction to $E$. Applying Proposition 3.1.4 to the map $k \rightarrow R$, we obtain an embedding of a Cohen ring $C_{k}$ for $k$ into $C_{E}$, the image of which contains $B_{0}$. Put

$$
\mathrm{O}_{k}=C_{k} \otimes_{W\left(\mathbb{F}_{q}\right)} \mathcal{O} \text {. }
$$

Then each $x \in \Gamma$ can be written formally as a sum $\sum_{i \in \mathbb{Z}} x_{i} t^{i}$ with $x_{i} \in \mathbb{O}_{k}$, such that for each $n$, the indices $i$ for which $v_{\cup_{k}}\left(x_{i}\right) \leq n$ are bounded below. For $n$ a nonnegative integer, we define the partial valuation function $v_{n}: \Gamma \rightarrow \mathbb{Z} \cup\{\infty\}$ by

$$
v_{n}(x)=\min \left\{i \in \mathbb{Z}: v_{\bigcirc_{k}}\left(x_{i}\right) \leq n\right\} .
$$

For $r>0$, put

$$
\Gamma^{r}=\left\{x \in \Gamma: \lim _{n \rightarrow \infty} v_{n}(x)+r n=\infty\right\} ;
$$

this is a subring of $\Gamma$. Put $\Gamma^{\dagger}=\bigcup_{r>0} \Gamma^{r}$; we may speak of $\nabla$-modules over $\Gamma^{\dagger}$ using the same definition as for $\Gamma$, using for the module of differentials

$$
\Omega_{\Gamma^{\dagger} / \mathscr{O}_{k}}^{1}=\bigoplus_{b \in B} \Gamma^{\dagger} d b
$$

(Here we are using the finiteness of the $p$-basis to avoid having to worry about a completion.) If $\phi$ is a Frobenius lift carrying $\Gamma^{\dagger}$ into itself, we may also define $\phi$-modules and $(\phi, \nabla)$-modules over $\Gamma^{\dagger}$ as before.

Definition 3.3.4. Since $O_{k} \subset \Gamma^{\dagger}$, we can identify a copy of $\mathcal{O}_{k}^{\text {unr }}$ inside $\left(\Gamma^{\dagger}\right)^{\text {unr }}$. Using this identification, put

$$
\widetilde{\Gamma}^{\dagger}=\widehat{\mathrm{O}_{k}^{\text {unr }}} \otimes_{\bigcirc_{k}^{\text {unr }}}\left(\Gamma^{\dagger}\right)^{\text {unr }} \subset \widehat{\Gamma^{\text {unr }}} .
$$

For $\rho$ a representation, put

$$
D^{\dagger}(\rho)=D(\rho) \cap\left(V(\rho) \otimes_{0} \widetilde{\Gamma}^{\dagger}\right)=\left(V(\rho) \otimes_{0} \widetilde{\Gamma}^{\dagger}\right)^{G_{E}} .
$$

Again, $D^{\dagger}(\rho)$ inherits a connection, and an action of any Frobenius lift $\phi$ acting on $\Gamma^{\dagger}$. Note that the natural map

$$
\left(D^{\dagger}(\rho) \otimes_{\Gamma^{\dagger}} \widetilde{\Gamma}^{\dagger}\right) \rightarrow\left(V(\rho) \otimes_{\odot} \widetilde{\Gamma}^{\dagger}\right)
$$

is always injective, and it is surjective if and only if $\rho$ has finite local monodromy.

The following is essentially [Tsuzuki 1998, Theorem 3.1.6].

Proposition 3.3.5. Let $\phi$ be a Frobenius lift on $\Gamma$ acting on $\Gamma^{\dagger}$. The base change functor from $(\phi, \nabla)$-modules over $\Gamma^{\dagger}$ to $(\phi, \nabla)$-modules over $\Gamma$ is fully faithful. 
Proof. Using internal Homs, we may rephrase this as follows: if $M$ is a $(\phi, \nabla)-$ module over $\Gamma^{\dagger}$, then

$$
(M \otimes \Gamma)^{\phi=1, \nabla=0} \subset M .
$$

In particular, it is sufficient to check this using only the $d t$ component of the connection. In this case, we may replace $\Gamma$ by the completion of $\Gamma\left[\phi^{-n}(b): b \in B_{0}, n \in\right.$ $\mathbb{Z}_{\geq 0}$, to get into the case where $R$ has perfect residue field. We may then conclude by applying [Tsuzuki 1996, 4.1.3].

The following is essentially [Tsuzuki 1998, Theorem 4.2.6].

Theorem 3.3.6. Let $\phi$ be a Frobenius lift on $\Gamma$ acting on $\Gamma^{\dagger}$. Then $D^{\dagger}$ and restriction induce equivalences between the following categories:

(a) representations with finite local monodromy;

(b) $(\phi, \nabla)$-modules over $\Gamma^{\dagger}$;

(c) $\nabla$-modules over $\Gamma^{\dagger}$ equipped with $\phi$-actions over $\Gamma$.

In particular, if a $\nabla$-module over $\Gamma^{\dagger}$ admits a $\phi$-action over $\Gamma$, that action is defined already over $\Gamma^{\dagger}$.

Proof. The functor from (a) to (b) is $D^{\dagger}$, while the functor from (b) to (c) is restriction. The functor from (c) back to (a) will be induced by $V$; once it is shown to be well-defined, it will be clear that the three functors compose to the identity starting from any point.

To obtain the functor from (c) to (a), we must prove that if $M$ is a $\nabla$-module over $\Gamma^{\dagger}$ such that $M \otimes \Gamma$ admits a compatible $\phi$-action, then the corresponding representation $V(M)$ has finite local monodromy. It suffices to check this after replacing $E$ by a finite extension, which can be chosen to ensure the existence of an isomorphism $(M / 2 p M) \otimes \Gamma \cong(\Gamma / 2 p \Gamma)^{m}$ of $\phi$-modules. In this case we claim that $V(M)$ is actually unramified; as in the proof of Proposition 3.3.5, it suffices to check this using only the $d t$ component of $\nabla$, and hence to reduce to the case of $R$ having perfect residue field. This case is treated by the proof of [Tsuzuki 1998, Proposition 5.2.1], but not by its statement (which requires a $\phi$-action over $\Gamma^{\dagger}$ ); for a literal citation, see [Kedlaya 2006a, Proposition 4.5.1].

3.4. $(\phi, \nabla)$-Modules over $\mathscr{R}$. Throughout this subsection, retain Hypothesis 3.3.2, and write $L$ for $\operatorname{Frac}\left(O_{k}\right)$ and $\mathscr{R}$ for $\mathscr{R}_{L}$. The choices made so far determine an embedding $\Gamma^{\dagger} \hookrightarrow \mathscr{R}_{L}$, and any Frobenius $\phi$ acting on $\Gamma^{\dagger}$ extends continuously to $\mathscr{R}_{L}$ (as in [Kedlaya 2004, Section 2]). We may thus define $\phi$-modules, $\nabla$-modules, and $(\phi, \nabla)$-modules over $\mathscr{R}$ using the same definitions as over $\Gamma$.

Remark 3.4.1. From a $\nabla$-module over $\mathscr{R}$, we may construct a $\nabla$-module on $A_{L}(\epsilon, 1)$ for some $\epsilon \in(0,1)$. The construction is unique in the following sense: any two such $\nabla$-modules become isomorphic on $A_{L}(\eta, 1)$ for some $\eta \in(0,1)$. 
Conversely, since any locally free sheaf on $A_{L}(\eta, 1)$ is freely generated by global sections (because $L$ is spherically complete; see for instance [Kedlaya 2005a, Theorem 3.14]), any $\nabla$-module on $A_{L}(\eta, 1)$ gives rise to a $\nabla$-module over $\mathscr{R}$.

Remark 3.4.1 is sufficient for the construction of the differential Swan conductor associated to a representation of finite local monodromy. However, for completeness, we record some related facts, including the analogue of the $p$-adic local monodromy theorem.

Lemma 3.4.2. Let $M$ be a $\phi$-module over $\Gamma^{\dagger}$ such that $M \otimes \mathscr{R}$ admits the structure of $a(\phi, \nabla)$-module. Then this structure is induced by $a(\phi, \nabla)$-module structure on $M$ itself, and so $M$ corresponds to a representation with finite local monodromy.

Proof. By [Kedlaya 2005b, Proposition 7.1.7], the action of $\partial / \partial t$ on $M \otimes \mathscr{R}$ acts on $M$ itself. Also, for any $b \in B_{0}$, we may change the $p$-basis by replacing $b$ by $b+t$, and then the same argument shows that the action of $\frac{\partial}{\partial t}+\frac{\partial}{\partial b}$ on $M \otimes \mathscr{R}$ acts on $M$ itself. (This is another instance of rotation in the sense of Remark 2.7.7.) We conclude that $\nabla$ itself acts on $M$, so we may apply Theorem 3.3.6 to conclude.

Definition 3.4.3. A $\phi$-module (resp. $(\phi, \nabla)$-module) $M$ over $\mathscr{R}$ is unit-root if it has the form $M_{0} \otimes \mathscr{R}$ for some $\phi$-module (resp. $(\phi, \nabla)$-module) $M_{0}$ over $\Gamma^{\dagger}$. By Lemma 3.4 .2 , a $(\phi, \nabla)$-module over $\mathscr{R}$ is unit-root if and only if its underlying $\phi$-module is unit-root.

Proposition 3.4.4. The base extension functor from the isogeny category of unitroot $\phi$-modules over $\Gamma^{\dagger}$ (i.e., $\phi$-modules over $\Gamma^{\dagger}\left[\frac{1}{p}\right]$ obtained by base extension from $\Gamma^{\dagger}$ ) to the category of unit-root $\phi$-modules over $\Re$ is an equivalence of categories.

Proof. This is [Kedlaya 2005b, Theorem 6.2.3].

Definition 3.4.5. Let $s=c / d$ be a rational number written in lowest terms. A $\phi$ module (resp. $(\phi, \nabla)$-module) $M$ over $\mathscr{R}$ is pure (or isoclinic) of slope $s$ if there exists a scalar $\lambda \in K^{*}$ of valuation $c$ such that the $\phi^{d}$-module (resp. $\left(\phi^{d}, \nabla\right)$-module) obtained from $M$ by twisting the $\phi^{d}$-action by $\lambda^{-1}$ is unit-root. In particular, by Theorem 3.3.6, the $\nabla$-module structure on $M$ corresponds to a representation with finite local monodromy after replacing $\mathcal{O}$ by a finite extension.

Theorem 3.4.6. Let $M$ be a $\phi$-module (resp. $(\phi, \nabla)$-module) $M$ over $\mathscr{R}$. Then there exists a unique filtration $0=M_{0} \subset M_{1} \subset \cdots \subset M_{l}=M$ of $M$ by saturated $\phi$-submodules (resp. $(\phi, \nabla)$-submodules) such that each quotient $M_{i} / M_{i-1}$ is pure of some slope $s_{i}$ as a $\phi$-module, and $s_{1}<\cdots<s_{l}$.

Proof. In the $\phi$-module case, this is [Kedlaya 2004, Theorem 6.10] or [Kedlaya 2005b, Theorem 6.4.1]. In the $(\phi, \nabla)$-module case, it suffices to check that the filtration of the underlying $\phi$-module is respected by $\nabla$. For this, we proceed as 
in [Kedlaya 2005b, Theorem 7.1.6]: for each derivation $\partial / \partial b$, we get a morphism of $\phi$-modules $M_{1} \rightarrow\left(M / M_{1}\right) \otimes \mathscr{R} d b$. The former is pure of slope $s_{1}$, whereas the latter admits a slope filtration in which each slope is strictly greater than $s_{1}$ (the slope of $\mathscr{R} d b$ being positive). By [Kedlaya 2005b, Proposition 4.6.4], that morphism is zero, proving that $M_{1}$ is respected by each derivation. Hence $M_{1}$ is a $(\phi, \nabla)$-submodule, and repeating the argument on $M / M_{1}$ yields the claim.

Remark 3.4.7. One may apply Theorem 3.3 .6 to each individual quotient of the filtration produced by Theorem 3.4.6. (Alternatively, one may project $\nabla$ onto the $d t$ component and directly invoke the $p$-adic local monodromy theorem; this allows the invocation of [André 2002] or [Mebkhout 2002] in place of [Kedlaya 2004].) It is an interesting question, which we have not considered, whether one can show that the category of $(\phi, \nabla)$-module $M$ over $\mathscr{R}_{L}$ is equivalent to a category of representations of $G_{E}$ times an algebraic group over Frac(O), as in [Kedlaya 2005a, Theorem 4.45].

3.5. Defining the differential Swan conductor. In order to use Theorem 3.3.6 to define the differential Swan conductor of a representation $\rho: G_{E} \rightarrow \mathrm{GL}(V)$ with finite local monodromy, we must check that the answer does not depend on the auxiliary choices we made along the way. (Note that the choice of $\phi$ does not matter: it is only used to define the Frobenius action on $D^{\dagger}(\rho)$, whereas only the connection is used to compute the conductor.)

Proposition 3.5.1. Suppose that $k$ admits a finite $p$-basis. For $\rho$ a representation with finite local monodromy, the isomorphism type of the $\nabla$-module $D^{\dagger}(\rho)$ does not depend on the choice of the Cohen ring $C_{E}$ or the lifted p-basis $B$.

Proof. By Proposition 3.1.4, the construction of $C_{E}$ is functorial in pairs $(E, \bar{B})$, where $\bar{B}$ is a $p$-basis of $E$. It thus suffices to check that if for $i=1,2, \bar{B}_{i}$ is a $p$-basis of $E$ consisting of a uniformizer $\bar{t}_{i}$ of $R$ and a lift $\overline{B_{i, 0}}$ to $R$ of a $p$-basis of $k$ over $k_{0}$, then the modules $D^{\dagger}(\rho)$ constructed using lifts of $\overline{B_{1}}$ and $\overline{B_{2}}$ are isomorphic, compatibly with some isomorphism of the underlying rings $\Gamma^{\dagger}$.

Let $\left(C_{E}, B_{1}\right)$ be a based Cohen ring lifting $\left(E, \overline{B_{1}}\right)$; write $C_{k, 1}, t_{1}$ instead of $C_{k}, t$. Define $B_{2,0}$ by choosing, for each $\bar{b} \in B_{2,0}$, a lift $b$ of $\bar{b}$ in $C_{k, 1} \llbracket t_{1} \rrbracket$. Then choose $t_{2}$ to be a lift of $\overline{t_{2}}$ belonging to $t_{1} C_{k, 1} \llbracket t_{1} \rrbracket$. We can then view $\left(C_{E}, B_{2}\right)$ as a based Cohen ring lifting $\left(E, \overline{B_{2}}\right)$, containing a Cohen ring $C_{k, 2}$ for $k$.

Since we used the same ring $C_{E}$ for both lifts, we may identify the two rings $\Gamma$. Although $C_{k, 1} \neq C_{k, 2}$ in general, we did ensure by construction that $C_{k, 1} \llbracket t_{1} \rrbracket=$ $C_{k, 2} \llbracket t_{2} \rrbracket$. Consequently, the two rings $\Gamma^{\dagger}$ constructed inside $\Gamma$ coincide, and we may identify the two copies of $\widetilde{\Gamma}^{\dagger}$. This gives an identification of the two modules $D^{\dagger}(\rho)$, as desired. 
Definition 3.5.2. Suppose to start that $k$ is finite over $k^{p}$, i.e., any $p$-basis of $k$ or of $E$ is finite. For $\rho: G_{E} \rightarrow \mathrm{GL}(V)$ a representation with finite local monodromy, with $V$ a finite dimensional 0 -module, we may now define the differential highest break, differential break multiset, and differential Swan conductor by constructing the $(\phi, \nabla)$-module $D^{\dagger}(\rho)$, for some Cohen ring $C_{E}$ and some lifted $p$-basis $B$, and computing the corresponding quantities associated to the underlying $\nabla$-module of $D^{\dagger}(\rho)$ tensored with the Robba ring $\mathscr{R}_{\mathrm{Frac}\left(\mathrm{O}_{k}\right)}$ (as in Remark 3.4.1). By Proposition 2.6.6 (to change the $p$-basis of $k$ ) and Proposition 3.5.1, this definition depends only on $\rho$ and not on any auxiliary choices. For general $k$, we may choose a finite subset $B_{1}$ of $B$ containing a lift $t$ of a uniformizer of $R$, project onto the span of the $d b$ for $b \in B_{1}$, and compute a conductor that way; this has the same effect as passing from $E$ to $E_{1}=\widehat{E_{0}}$, where

$$
E_{0}=E\left(\bar{b}^{1 / p^{n}}: b \in B \backslash B_{1}, n \in \mathbb{N}_{0}\right) .
$$

We define the differential Swan conductor of $\rho$ in this case to be the supremum over all choices of $B$ and $B_{1}$; it will turn out to be finite (Corollary 3.5.7) and hence integral by Theorem 2.8.2.

Definition 3.5.3. Let $E^{\prime} / E$ be a finite separable extension, let $\bar{B}$ be a $p$-basis of $E$ containing a uniformizer $\bar{t}$ of $E$, and put $\overline{B_{0}}=\bar{B} \backslash\{\bar{t}\}$. We say a subset $\overline{B_{2}}$ of $\overline{B_{0}}$ is a rectifying set for $E^{\prime} / E$ if, putting $E_{2}=\widehat{E_{0}}$ for

$$
E_{0}=E\left(\bar{b}^{1 / p^{n}}: \bar{b} \in \overline{B_{2}}, n \in \mathbb{N}_{0}\right),
$$

the extension $\left(E^{\prime} \otimes_{E} E_{2}\right) / E_{2}$ has separable residue field extension. Beware that it is not enough for the residue field of $E_{2}$ to contain the perfect closure of $k$ in the residue field of $E^{\prime}$. For instance, if $p>2, \overline{b_{1}}, \overline{b_{2}} \in \bar{B}$, and

$$
E^{\prime}=E[z] /\left(z^{p}-z-\bar{b}_{1} \bar{t}^{-2 p}-\bar{b}_{2} \bar{t}^{-p}\right),
$$

then $E^{\prime}$ has residue field $k\left(\overline{b_{1}} 1 / p\right)$, but $\overline{B_{2}}=\left\{\overline{b_{1}}\right\}$ is not a rectifying set because the residue field of $E^{\prime} \otimes_{E} E_{2}$ contains $\bar{b}_{2}^{1 / p}$.

Lemma 3.5.4. With notation as in Definition 3.5.3, $\bar{B}$ contains a finite rectifying set for $E^{\prime} / E$.

Proof. Use $\bar{B}$ to embed $k$ into $E$. By induction on the degree of $E^{\prime} / E$, we may reduce to the case of an Artin-Schreier extension

$$
E^{\prime}=E[z] /\left(z^{p}-z-a_{n} \bar{t}^{-n}-\cdots-a_{1} \bar{t}^{-1}-a_{0}\right)
$$

with $a_{i} \in k$. In this case, pick any $N \in \mathbb{N}_{0}$ with $p^{N}>n$, and write each $a_{i}$ as a $k^{p^{N}}$-linear combination of products of powers of elements of $\overline{B_{0}}$. Only finitely many elements of $\overline{B_{0}}$ get used; those form a rectifying set. 
Proposition 3.5.5. Suppose that there exists a finite separable extension $E^{\prime}$ of $E$ whose residue field is separable over $k$, such that $\rho$ is unramified on $G_{E^{\prime}}$. Then the differential break multiset and Swan conductor of a representation $\rho$ with finite local monodromy can be computed with respect to $\{t\}$, and it agrees with the usual break multiset and Swan conductor.

Proof. It suffices to consider $\rho$ irreducible and check equality for the highest breaks. Note that the usual highest break is insensitive to further residue field extension, because it can be computed using Herbrand's formalism as in [Serre 1979, Chapter IV]. It thus agrees with the differential highest break computed with respect to $\{t\}$ : namely, this claim reduces to the case where $k$ is perfect, for which see [Kedlaya 2005a, Theorem 5.23] and references thereafter.

It remains to show that for any $B_{1}, \partial / \partial t$ must be eventually dominant. Suppose the contrary, and pick $b \in B_{1} \backslash\{t\}$ such that $\partial / \partial b$ is eventually dominant. By a tame base change (invoking Proposition 2.6.5), we can force the gap between the differential highest breaks computed with respect to $B_{1}$ and with respect to $\{t\}$ to be greater than 1; then a rotation as in Lemma 2.7.10 sending $b$ to $b+t$ raises the differential highest break computed with respect to $\{t\}$. But that contradicts the previous paragraph: both before and after rotation, the differential highest break computed with respect to $\{t\}$ must coincide with the usual highest break.

We deduce that $\partial / \partial t$ is eventually dominant, proving the claim.

Corollary 3.5.6. In the notation of Definition 3.5.2, suppose that there exists a finite separable extension $E^{\prime}$ of $E$ such that $\rho$ is unramified on $G_{E^{\prime}}$, and that the image of $B_{1} \backslash\{t\}$ in $E$ is a rectifying set for $E^{\prime} / E$. Then the differential Swan conductor of $\rho$ computed using $\left(B \backslash B_{1}\right) \cup\{t\}$ is equal to that computed using $t$.

Corollary 3.5.7. In the notation of Definition 3.5.2, suppose that there exists a finite separable extension $E^{\prime}$ of $E$ such that $\rho$ is unramified on $G_{E^{\prime}}$, and that the image of $B_{1} \backslash\{t\}$ in $E$ is a rectifying set for $E^{\prime} / E$. Then the differential Swan conductor of $\rho$ is equal to that computed using $B_{1}$.

For completeness, we record the following observations.

Theorem 3.5.8. The differential Swan conductor of any representation with finite local monodromy is a nonnegative integer.

Proof. By Lemma 3.5.4 and Corollary 3.5.7, the conductor can be computed using a finite set $B_{1}$; we may thus apply Theorem 2.8.2.

Theorem 3.5.9. Let $E^{\prime}$ be a tamely ramified extension of $E$ of ramification degree $m$. Let $\rho$ be a representation of $G_{E}$ with finite local monodromy, and let $\rho^{\prime}$ be the restriction of $\rho$ to $G_{E^{\prime}}$. Then $\operatorname{Swan}\left(\rho^{\prime}\right)=m \operatorname{Swan}(\rho)$.

Proof. Apply Proposition 2.6.5. 
Example 3.5.10. As an example, consider a nontrivial character of the ArtinSchreier extension $E[z] /\left(z^{p}-z-\bar{x}\right)$. The corresponding differential module will be a Dwork isocrystal, i.e., a rank one $\nabla$-module with generator $\mathbf{v}$ such that

$$
\nabla(\mathbf{v})=\pi \mathbf{v} \otimes d x,
$$

for $x$ some lift of $\bar{x}$ and $\pi$ a ( $p-1)$-st root of $-p$. One computes that the differential Swan conductor for this character is equal to the least integer $m \geq 0$ such that $v_{E}\left(x-y^{p}+y\right) \geq-m$ for all $y \in E$. This agrees with the definition given by [Kato $1989]$ of the Swan conductor of a character; note that the conductor is allowed to be divisible by $p$ if and only if $k$ is imperfect.

Remark 3.5.11. Given a representation $\rho: G_{E} \rightarrow \mathrm{GL}(V)$, where $V$ is a finite dimensional Frac(O)-vector space, we may define a differential Swan conductor for it by picking a $\rho$-stable 0 -lattice of $V$ and proceeding as in Definition 3.5.2. Changing the lattice will not change the resulting $\nabla$-module over $\mathscr{R}_{\mathrm{Frac}\left(\mathscr{O}_{k}\right)}$, so we get a well-defined numerical invariant of $\rho$ also.

Defining conductors for Galois representations is tantamount to filtering the Galois group; let us now make this explicit.

Definition 3.5.12. Put $G_{E}^{0}=I_{E}$. For $r>0$, let $R_{r}$ be the set of representations $\rho$ with highest break less than $r$, and put

$$
G_{E}^{r}=\bigcap_{\rho \in R_{r}}\left(I_{E} \cap \operatorname{ker}(\rho)\right) .
$$

Note that $\rho \in R_{r}$ if and only if $G_{E}^{r} \subseteq I_{E} \cap \operatorname{ker}(\rho)$; this reduces to the fact that $R_{r}$ is stable under tensor product and formation of subquotients. We call $G_{E}^{r}$ the differential upper numbering filtration on $G_{E}$. Write $G_{E}^{r+}$ for the closure of $\bigcup_{s>r} G_{E}^{s}$; note that $G_{E}^{r}=G_{E}^{r+}$ for $r$ irrational, because differential highest breaks are always rational numbers.

As in the perfect residue field case, the graded pieces of the upper numbering filtration are particularly simple.

Theorem 3.5.13. For $r>0$ rational, $G_{E}^{r} / G_{E}^{r+}$ is abelian and killed by $p$.

Proof. Let $E^{\prime}$ be a finite Galois extension of $E$ with $\operatorname{Gal}\left(E^{\prime} / E\right)=G$; then we obtain an induced filtration on $G$ by taking $G^{r}$ to be the image of $G_{E}^{r}$ under the surjection $G_{E} \rightarrow G$. It suffices to check that $G^{r} / G^{r+}$ is abelian and killed by $p$; moreover, we may quotient further to reduce to the case where $G^{r+}$ is the trivial group but $G^{r}$ is not. Let $\rho$ be the regular representation of $G$; then $\rho$ has highest break $r$. Let $S$ be the set of irreducible constituents of $\rho$ of highest break strictly less than $r$; we are then trying to show that the intersection of $\operatorname{ker}(\psi) \subseteq G$ over all $\psi \in S$ is an elementary abelian $p$-group. 
By Corollary 3.5.7, we may reduce to the case where the lifted $p$-basis $B$ of Hypothesis 3.3.2 is finite; put $B_{0}=\left\{b_{1}, \ldots, b_{n}\right\}$. By making a tame base change, we can force all nonzero ramification breaks to be greater than 1. By another base change (passing from Frac $C_{E}$ to the completion of $C_{E}\left(v_{1}, \ldots, v_{n}\right)$ for the $(1, \ldots, 1)$-Gauss norm), we can add extra elements $v_{1}, \ldots, v_{n}$ to $B$, then perform the operation described in Proposition 2.7.11. Each nonzero ramification break $m$ before the operation corresponds to the break $p m-p+1$ afterwards, so the desired result may be checked afterwards. But now $\partial / \partial t$ is dominant on every irreducible component of $\rho$, so we may reduce to the case of perfect residue field and (by Proposition 3.5.5) the usual upper numbering filtration. In this case, the claim is standard: it follows from the fact that the upper numbering filtration can be constructed by renumbering the lower numbering filtration [Serre 1979, Section IV.3], for which the claim is easy to check [Serre 1979, Section IV.2, Corollary 3 of Proposition 7].

Remark 3.5.14. Note that the definition of the differential Swan conductor of a representation is invariant under enlarging 0 , because the differential Swan conductor of a $\nabla$-module is invariant under enlarging the constant field $K$.

3.6. Reconciliation questions. By introducing a numerical invariant of representations and calling it a conductor, one begs various reconciliation questions with other definitions. To begin with, it is known (and was a motivation of our construction) that in the traditional case of a perfect residue field, one computes the right numbers; see Proposition 3.5.5.

In the general case, there is a definition of the "logarithmic conductor" due to [Abbes and Saito 2002; Abbes and Saito 2003]. Following [Matsuda 2004], one is led to ask the following.

Question 3.6.1. For $\rho$ a representation with finite local monodromy, does the differential Swan conductor agree with the Abbes-Saito logarithmic conductor in equal characteristic?

It is easy to check the affirmative answer for Artin-Schreier characters. An affirmative answer in the general case would have the beneficial consequence of verifying the Hasse-Arf theorem for the Abbes-Saito conductor in equal characteristic. Some progress on this question has been made recently by Bruno Chiarellotto and Andrea Pulita, and independently by Liang Xiao.

One might also try to reconcile our definition with conductors for Galois representations over a two-dimensional local field, as in [Zhukov 2000; Zhukov 2003]. In order to formulate a precise question, it may be easiest to pass to the context of considering a representation of the étale fundamental group of a surface and computing its conductor along different boundary divisors. Indeed, this will be the point of view of the sequel to this paper. 
There is also a construction of Artin conductors in the imperfect residue field case due to [Borger 2004], by passing from $E$ to a certain extension which is universal for the property of having perfect residue field. Borger's construction does not behave well with respect to tame base extension, but one should get a better invariant by forcing such good behavior (i.e., constructing a logarithmic analogue of Borger's conductor). Indeed, we expect the following.

Conjecture 3.6.2. For $\rho$ a representation with finite local monodromy, for $m$ a positive integer, let $E_{m}$ be an extension of $E$ which is tamely ramified of tame degree $m$. Let $b^{\prime}\left(E_{m}\right)$ be Borger's Artin conductor of the restriction of $\rho$ to $G_{E_{m}}$. Then the limsup of $m^{-1} b^{\prime}\left(E_{m}\right)$ as $m \rightarrow \infty$ equals the differential Swan conductor of $\rho$.

Since the Abbes-Saito construction also works in mixed characteristic, one may also be interested in reconciling it there with a differential construction. For more on this possibility, see the next subsection.

3.7. Comments on mixed characteristic. It would be interesting to extend the constructions in this paper to the case where $R$ has mixed characteristics. The analogue of the passage from Galois representations to $\nabla$-modules is given by $p$ adic Hodge theory, specifically via the theory of $(\phi, \Gamma)$-modules over the Robba ring, as in the work of Fontaine, Cherbonnier-Colmez, Berger, et al.

In that context, when $R$ has perfect residue field, Colmez [2003] has given a recipe for reading off the Swan conductor of de Rham representations from the associated $(\phi, \Gamma)$-module . One would like to reformulate this recipe via Berger's construction of the Weil-Deligne representation, which converts the $(\phi, \Gamma)$-module into a $(\phi, \nabla)$-module over $\mathscr{R}$ [Berger 2002]; however, it is not immediately clear how to do this. The fact that this might even be possible is suggested by work of Marmora [2004], who gives a direct comparison with differential Swan conductors, but only for the Swan conductor of a representation over the maximal $p$-cyclotomic extension of a given $p$-adic field.

If one can indeed give a differential definition of the usual Swan conductor of a de Rham representation in the perfect residue field, then it seems likely one can make a differential definition in the imperfect residue field case. Indeed, the construction of $(\Phi, \Gamma)$-modules has already been generalized to this setting by Morita [2005]. If one can do all this, then one will again encounter the question of reconciliation with the Abbes-Saito constructions; however, it is not clear whether in this case the Hasse-Arf theorem would be any easier on the differential side than on the Abbes-Saito side. 


\section{References}

[Abbes and Saito 2002] A. Abbes and T. Saito, "Ramification of local fields with imperfect residue fields", Amer. J. Math. 124:5 (2002), 879-920. MR 2003m:11196 Zbl 1084.11064

[Abbes and Saito 2003] A. Abbes and T. Saito, "Ramification of local fields with imperfect residue fields. II”, Doc. Math. Extra Vol. (2003), 5-72. MR 2005g:11231 Zbl 02028830

[Amice 1975] Y. Amice, Les nombres p-adiques, SUP: Le Mathématicien 14, Presses Universitaires de France, Paris, 1975. MR 56 \#5510 Zbl 0313.12104

[André 2002] Y. André, "Filtrations de type Hasse-Arf et monodromie p-adique", Invent. Math. 148:2 (2002), 285-317. MR 2003k:12011 Zbl 1081.12003

[Beilinson et al. 2002] A. Beilinson, S. Bloch, and H. Esnault, " $\epsilon$-factors for Gauss-Manin determinants", Mosc. Math. J. 2:3 (2002), 477-532. MR 2004m:14011 Zbl 1061.14010

[Berger 2002] L. Berger, "Représentations p-adiques et équations différentielles", Invent. Math. 148:2 (2002), 219-284. MR 2004a:14022 Zbl 01777245

[Berkovich 1990] V. G. Berkovich, Spectral theory and analytic geometry over non-Archimedean fields, Mathematical Surveys and Monographs 33, American Mathematical Society, Providence, RI, 1990. MR 91k:32038 Zbl 0715.14013

[Borger 2004] J. M. Borger, "Conductors and the moduli of residual perfection", Math. Ann. 329:1 (2004), 1-30. MR 2005c:11146 Zbl 02123992

[Bourbaki 1983] N. Bourbaki, Algèbre commutative, Chapitres 8 et 9, Masson, Paris, 1983. MR 86j:13001 Zbl 0579.13001

[Christol and Dwork 1994] G. Christol and B. Dwork, "Modules différentiels sur des couronnes", Ann. Inst. Fourier (Grenoble) 44:3 (1994), 663-701. MR 96f:12008 Zbl 0859.12004

[Christol and Mebkhout 2000] G. Christol and Z. Mebkhout, "Sur le théorème de l'indice des équations différentielles p-adiques. III", Ann. of Math. (2) 151:2 (2000), 385-457. MR 2001k:12014 Zbl 1078.12500

[Christol and Mebkhout 2001] G. Christol and Z. Mebkhout, "Sur le théorème de l'indice des équations différentielles p-adiques. IV”, Invent. Math. 143:3 (2001), 629-672. MR 2002d:12005 Zbl 1078.12501

[Cohen 1946] I. S. Cohen, "On the structure and ideal theory of complete local rings", Trans. Amer. Math. Soc. 59 (1946), 54-106. MR 7,509h Zbl 0060.07001

[Colmez 2003] P. Colmez, “Conducteur d'Artin d'une représentation de de Rham”, preprint, 2003, Available at http://www.math.jussieu.fr/ colmez/swan.pdf.

[Dwork et al. 1994] B. Dwork, G. Gerotto, and F. J. Sullivan, An introduction to G-functions, Annals of Mathematics Studies 133, Princeton University Press, Princeton, NJ, 1994. MR 96c:12009 Zbl 0830.12004

[Fontaine 1990] J.-M. Fontaine, "Représentations p-adiques des corps locaux. I", pp. 249-309 in The Grothendieck Festschrift, Vol. II, edited by P. Cartier et al., Progr. Math. 87, Birkhäuser, Boston, 1990. MR 92i:11125 Zbl 0743.11066

[Fresnel and van der Put 2004] J. Fresnel and M. van der Put, Rigid analytic geometry and its applications, Progress in Mathematics 218, Birkhäuser, Boston, 2004. MR 2004i:14023 Zbl 02043955

[Kato 1989] K. Kato, "Swan conductors for characters of degree one in the imperfect residue field case", pp. 101-131 in Algebraic K-theory and algebraic number theory (Honolulu, 1987), edited by M. R. Stein and R. K. Dennis, Contemp. Math. 83, Amer. Math. Soc., Providence, RI, 1989. MR 90g:11164 Zbl 0716.12006 
[Kedlaya 2004] K. S. Kedlaya, “A p-adic local monodromy theorem”, Ann. of Math. (2) 160:1 (2004), 93-184. MR 2005k:14038 Zbl 1088.14005

[Kedlaya 2005a] K. S. Kedlaya, "Local monodromy of $p$-adic differential equations: an overview", Int. J. Number Theory 1:1 (2005), 109-154. MR 2006g:12013 Zbl 1107.12005

[Kedlaya 2005b] K. S. Kedlaya, "Slope filtrations revisited", Doc. Math. 10 (2005), 447-525. MR 2007c:14016 Zbl 1081.14028

[Kedlaya 2006a] K. S. Kedlaya, "The $p$-adic local monodromy theorem for fake annuli", preprint, 2006. math.NT/0507496

[Kedlaya 2006b] K. S. Kedlaya, "Semistable reduction for overconvergent $F$-isocrystals, III: Local semistable reduction at monomial valuations", preprint, 2006. math.NT/0609645

[Kedlaya 2007] K. S. Kedlaya, "Semistable reduction for overconvergent $F$-isocrystals, I: Unipotence and logarithmic extensions", Compos. Math. 143 (2007), 1164-1212.

[Lazard 1962] M. Lazard, "Les zéros des fonctions analytiques d'une variable sur un corps valué complet”, Inst. Hautes Études Sci. Publ. Math. 14 (1962), 47-75. MR 27 \#2497 Zbl 1081.14028

[Marmora 2004] A. Marmora, "Irrégularité et conducteur de Swan p-adiques", Doc. Math. 9 (2004), 413-433. MR 2005i:11169 Zbl 1071.11065

[Matsuda 2004] S. Matsuda, "Conjecture on Abbes-Saito filtration and Christol-Mebkhout filtration", pp. 845-856 in Geometric aspects of Dwork theory. Vol. I, II, edited by A. Adolphson et al., Walter de Gruyter GmbH \& Co. KG, Berlin, 2004. MR 2005g:11233 Zbl 02127988

[Mebkhout 2002] Z. Mebkhout, "Analogue $p$-adique du théorème de Turrittin et le théorème de la monodromie $p$-adique”, Invent. Math. 148:2 (2002), 319-351. MR 2003k:14018 Zbl 1071.12004

[Morita 2005] K. Morita, "Galois cohomology of $p$-adic fields via $\left(\Phi, \Gamma_{K}\right)$-module in the imperfect residue field case", preprint, 2005. math.NT/0507009

[Ore 1933] O. Ore, “Theory of non-commutative polynomials", Ann. of Math. (2) 34:3 (1933), 480508. MR MR1503119 JFM 59.0925.01

[Robba 1980] P. Robba, "Lemmes de Hensel pour les opérateurs différentiels. Application à la réduction formelle des équations différentielles", Enseign. Math. (2) 26:3-4 (1980), 279-311 (1981). MR 82k:12022 Zbl 0466.12014

[Robba and Christol 1994] P. Robba and G. Christol, Équations différentielles p-adiques: Applications aux sommes exponentielles, Hermann, Paris, 1994. MR 97g:12005 Zbl 0868.12006

[Schneider 2002] P. Schneider, Nonarchimedean functional analysis, Springer Monographs in Mathematics, Springer, Berlin, 2002. MR 2003a:46106 Zbl 0998.46044

[Serre 1979] J.-P. Serre, Local fields, Graduate Texts in Mathematics 67, Springer, New York, 1979. MR 82e:12016 Zbl 0423.12016

[Tsuzuki 1996] N. Tsuzuki, "The overconvergence of morphisms of étale $\phi$ - $\nabla$-spaces on a local field”, Compositio Math. 103:2 (1996), 227-239. MR 97m:14024 Zbl 0872.14015

[Tsuzuki 1998] N. Tsuzuki, "Finite local monodromy of overconvergent unit-root $F$-isocrystals on a curve", Amer. J. Math. 120:6 (1998), 1165-1190. MR 99k:14038 Zbl 0943.14007

[Whitney 2002] W. Whitney, Functorial Cohen rings, Ph.D. thesis, University of California, Berkeley, 2002, Available at http://math.berkeley.edu/ whitney/dissertation/dissertation.pdf.

[Zhukov 2000] I. Zhukov, "An approach to higher ramification theory", pp. 143-150 in Invitation to higher local fields (Münster, 1999), edited by I. Fesenko and M. Kurihara, Geom. Topol. Monogr.

3, Geom. Topol. Publ., Coventry, 2000. MR 2001j:11110 Zbl 1008.11045

[Zhukov 2003] I. B. Zhukov, "On ramification theory in the case of an imperfect residue field", Mat. Sb. 194:12 (2003), 3-30. MR 2005e:11158 Zbl 1063.11046 
Communicated by Hélène Esnault

Received 2007-02-12

Revised 2007-08-13

Accepted 2007-09-17

kedlaya@mit.edu

Department of Mathematics, Massachusetts Institute of Technology, 77 Massachusetts Avenue, Cambridge, MA 02139

http://math.mit.edu/ kedlaya 


\title{
$L_{\infty}$ structures on mapping cones
}

\author{
Domenico Fiorenza and Marco Manetti
}

\begin{abstract}
We show that the mapping cone of a morphism of differential graded Lie algebras, $\chi: L \rightarrow M$, can be canonically endowed with an $L_{\infty}$-algebra structure which at the same time lifts the Lie algebra structure on $L$ and the usual differential on the mapping cone. Moreover, this structure is unique up to isomorphisms of $L_{\infty}$-algebras.
\end{abstract}

\section{Introduction}

There are several cases where the tangent and obstruction spaces of a deformation theory are the cohomology groups of the mapping cone of a morphism $\chi: L \rightarrow M$ of differential graded Lie algebras. It is therefore natural to ask if there exists a canonical differential graded Lie algebra structure on the complex $\left(C_{\chi}, \delta\right)$, where

$$
C_{\chi}=\bigoplus C_{\chi}^{i}, \quad C_{\chi}^{i}=L^{i} \bigoplus M^{i-1}, \quad \delta(l, m)=(d l, \chi(l)-d m),
$$

such that the projection $C_{\chi} \rightarrow L$ is a morphism of differential graded Lie algebras.

In general we cannot expect the existence of a Lie structure. In fact the canonical bracket

$$
\begin{aligned}
l_{1} \otimes l_{2} & \mapsto\left[l_{1}, l_{2}\right], \quad m_{1} \otimes l_{2} \mapsto \frac{1}{2}\left[m_{1}, \chi\left(l_{2}\right)\right], \\
l_{1} \otimes m_{2} & \mapsto \frac{1}{2}(-1)^{\operatorname{deg}\left(l_{1}\right)}\left[\chi\left(l_{1}\right), m_{2}\right], \quad m_{1} \otimes m_{2} \mapsto 0
\end{aligned}
$$

satisfies the Leibniz rule with respect to the differential $\delta$ but not the Jacobi identity. However, the Jacobi identity for this bracket holds up to homotopy, and so we can look for the weaker requirement of a canonical $L_{\infty}$ structure on $C_{\chi}$.

More precisely, let $\mathbb{K}$ be a fixed characteristic zero base field, denote by DG the category of differential graded vector spaces, by DGLA the category of differential graded Lie algebras, by $\mathbf{L}_{\infty}$ the category of $L_{\infty}$ algebras and by $\mathbf{D G L A}{ }^{2}$ the

MSC2000: primary 17B70; secondary 13D10.

Keywords: differential graded Lie algebra, symmetric coalgebra, $L_{\infty}$-algebra, functor of Artin ring. 
category of morphisms in DGLA. The four functors,

$$
\begin{array}{cl}
\text { DGLA } \rightarrow \mathbf{L}_{\infty} & \text { by natural inclusion, } \\
\mathbf{L}_{\infty} \rightarrow \text { DG } & \text { by forgetting higher brackets, } \\
\text { DGLA }^{2} \rightarrow \text { DG } & \text { by }\{L \stackrel{\chi}{\longrightarrow} M\} \mapsto C_{\chi}, \\
\text { DGLA }^{\rightarrow} \text { DGLA }^{2} & \text { by } L \mapsto\{L \rightarrow 0\},
\end{array}
$$

give a commutative diagram

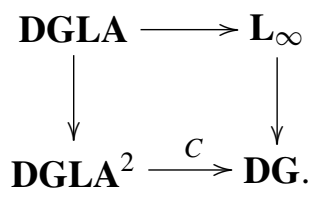

Theorem 1. There exists a functor $\widetilde{C}: \mathbf{D G L A}^{2} \rightarrow \mathbf{L}_{\infty}$ making the diagram

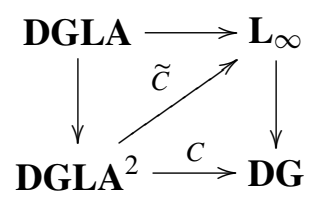

commutative.

Moreover, the functor $\widetilde{C}$ is essentially unique, that is, if $\mathscr{F}: \mathbf{D G L A}^{2} \rightarrow \mathbf{L}_{\infty}$ has the same properties, then for every morphism $\chi$ of differential graded Lie algebras, the $L_{\infty}$-algebra $\mathscr{F}(\chi)$ is (noncanonically) isomorphic to $\widetilde{C}(\chi)$.

The $L_{\infty}$ structure $\widetilde{C}(\chi)$ on the mapping cone of a DGLA morphism $\chi: L \rightarrow M$ is actually a particular case of a more general construction of an $L_{\infty}$ structure on the total complex of a semicosimplicial DGLA. More precisely, the category DGLA $^{2}$ of morphisms of DGLAs can be seen as a full subcategory of the category DGLA $^{\Delta_{\text {mon }}}$ of semicosimplicial DGLAs via the functor

$$
\{L \stackrel{\chi}{\rightarrow} M\} \rightsquigarrow\{L \stackrel{0}{\underset{\chi}{\rightleftarrows}} M \rightrightarrows 0 \Longrightarrow
$$

and we have a commutative diagram

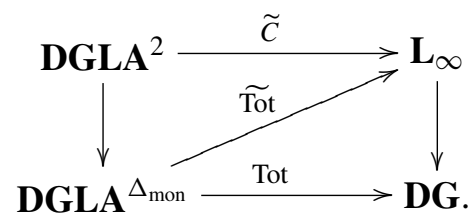

The functor $\widetilde{C}$ can be explicitly described. The linear term of the $L_{\infty}$-algebra $\widetilde{C}(\chi)$ is by construction the differential $\delta$ on $C_{\chi}$, and the quadratic part which turns 
out to coincide with the naive bracket described at the beginning of the Introduction. An explicit expression for the higher brackets is given in Theorem 5.2.

The second main result of this paper is to prove that the deformation functor $\operatorname{Def}_{\widetilde{C}(\chi)}$ associated with the $L_{\infty}$ algebra $\widetilde{C}(\chi)$ is isomorphic to the functor $\operatorname{Def}_{\chi}$ defined in [Manetti 2005].

Given $\chi: L \rightarrow M$, it defines a functor $\operatorname{Def}_{\chi}:$ Art $\rightarrow$ Set, with Art the category of local Artinian $\mathbb{K}$-algebras with residue field $\mathbb{K}$,

$\operatorname{Def}_{\chi}(A)$

$$
=\frac{\left\{\left(x, e^{a}\right) \in\left(L^{1} \otimes \mathfrak{m}_{A}\right) \times \exp \left(M^{0} \otimes \mathfrak{m}_{A}\right) \mid d x+\frac{1}{2}[x, x]=0, e^{a} * \chi(x)=0\right\}}{\text { gauge equivalence }}
$$

where $*$ denotes the gauge action in $M$, and $\left(l_{0}, e^{m_{0}}\right)$ is defined to be gauge equivalent to $\left(l_{1}, e^{m_{1}}\right)$ if there exists $(a, b) \in\left(L^{0} \bigoplus M^{-1}\right) \otimes \mathfrak{m}_{A}$ such that

$$
l_{1}=e^{a} * l_{0}, \quad e^{m_{1}}=e^{d b} e^{m_{0}} e^{-\chi(a)} .
$$

Theorem 2. With the notation above, for every morphism of differential graded Lie algebras, $\chi: L \rightarrow M$, we have

$$
\operatorname{Def}_{\widetilde{C}(\chi)} \simeq \operatorname{Def}_{\chi} .
$$

The importance of Theorem 2 lies in that it allows one to study the functors $\operatorname{Def}_{\chi}$, which are often naturally identified with geometrically defined functors, using the whole machinery of $L_{\infty}$-algebras. In particular this gives, under some finiteness assumption, the construction and the homotopy invariance of the Kuranishi map [Fukaya 2003; Goldman and Millson 1990; Kontsevich 2003], as well as the local description of the corresponding extended moduli spaces.

Keywords and general notation. We assume that the reader is familiar with the notion and main properties of differential graded Lie algebras and $L_{\infty}$-algebras (we refer to [Fukaya 2003; Grassi 1999; Kontsevich 2003; Lada and Markl 1995; Lada and Stasheff 1993; Manetti 2004b] as the introduction of such structures); however the basic definitions are recalled in this paper in order to fix notation and terminology.

For the whole paper, $\mathbb{K}$ is a fixed field of characteristic 0 and Art is the category of local Artinian $\mathbb{K}$-algebras with residue field $\mathbb{K}$. For $A \in$ Art we denote by $\mathfrak{m}_{A}$ the maximal ideal of $A$.

\section{Conventions on graded vector spaces}

In this paper we will work with $\mathbb{Z}$-graded vector spaces. We write a graded vector space as $V=\bigoplus_{n \in \mathbb{Z}} V^{n}$, and call $V^{n}$ the degree $n$ component of $V$; an element $v$ 
of $V^{n}$ is called a degree $n$ homogeneous element of $V$. The shift functor is defined as $(V[k])^{i}:=V^{i+k}$. We say that a linear map $\varphi: V \rightarrow W$ is a degree $k$ map if it is a morphism $V \rightarrow W[k]$, that is, if it is a collection of linear maps $\varphi^{n}: V^{n} \rightarrow W^{n+k}$. The set of degree $k$ liner maps from $V$ to $W$ will be denoted $\operatorname{Hom}^{k}(V, W)$.

Graded vector spaces form a symmetric tensor category with

$$
(V \otimes W)^{k}=\bigoplus_{i+j=k} V^{i} \otimes W^{j},
$$

and $\sigma_{V, W}: V \otimes W \rightarrow W \otimes V$ given by $\sigma(v \otimes w):=(-1)^{\operatorname{deg}(v) \cdot \operatorname{deg}(w)} w \otimes v$ on the homogeneous elements. We adopt the convention according to which degrees are "shifted on the left". By this we mean that we have a natural identification, called the suspension isomorphism, $V[1] \simeq \mathbb{K}[1] \otimes V$ where $\mathbb{K}[1]$ denotes the graded vector space consisting of the field $\mathbb{K}$ concentrated in degree -1 . With this convention, the canonical isomorphism is

$$
V \otimes \mathbb{K}[1] \simeq V[1], \quad v \otimes 1_{[1]} \mapsto(-1)^{\operatorname{deg}(v)} v_{[1]} .
$$

More in general we have the following decalage isomorphism

$$
\begin{aligned}
V_{1}[1] & \otimes \cdots \otimes V_{n}[1] \stackrel{\sim}{\rightarrow}\left(V_{1} \otimes \cdots \otimes V_{n}\right)[n], \\
v_{1[1]} & \otimes \otimes v_{n[1]} \mapsto(-1)^{\sum_{i=1}^{n}(n-i) \cdot \operatorname{deg} v_{i}}\left(v_{1} \otimes \cdots \otimes v_{n}\right)_{[n]} .
\end{aligned}
$$

Since graded vector spaces form a symmetric category, for any graded vector space $V$ and any positive integer $n$ we have a canonical representation of the symmetric group $S_{n}$ on $\otimes^{n} V$. The space of coinvariants for this action is called the $n$-th symmetric power of $V$ and is denoted by $\odot^{n} V$. Twisting the canonical representation of $S_{n}$ on $\otimes^{n} V$ by the alternating character $\sigma \mapsto(-1)^{\sigma}$ and taking the coinvariants one obtains the $n$-th antisymmetric (or exterior) power of $V$, denoted by $\bigwedge^{n} V$. By the naturality of the decalage isomorphism, we have a canonical isomorphism

$$
\bigodot^{n}(V[1]) \stackrel{\sim}{\rightarrow}\left(\bigwedge^{n} V\right)[n] .
$$

Remark 1.1. Using the natural isomorphisms

$$
\operatorname{Hom}^{i}(V, W[l]) \simeq \operatorname{Hom}^{i+l}(V, W)
$$

and the decalage isomorphism, we obtain the natural identifications

$$
\begin{gathered}
\operatorname{dec}: \operatorname{Hom}^{i}\left(\bigwedge^{k} V, W\right) \stackrel{\sim}{\rightarrow} \operatorname{Hom}^{i+k-1}\left(\bigodot^{k}(V[1]), W[1]\right), \\
\operatorname{dec}(f)\left(v_{1[1]} \odot \cdots \odot v_{k[1]}\right)=(-1)^{k i+\sum_{j=1}^{k}(k-j) \cdot \operatorname{deg}\left(v_{j}\right)} f\left(v_{1} \wedge \cdots \wedge v_{k}\right)_{[1]} .
\end{gathered}
$$




\section{Differential graded Lie algebras and $L_{\infty}$-algebras}

A differential graded Lie algebra (DGLA) is a Lie algebra in the category of graded vector spaces, endowed with a compatible degree 1 differential. Via the decalage isomorphisms one can look at the Lie bracket of a DGLA $V$ as a morphism

$$
q_{2} \in \operatorname{Hom}^{1}(V[1] \odot V[1], V[1]), \quad q_{2}\left(v_{[1]} \odot w_{[1]}\right)=(-1)^{\operatorname{deg}(v)}[v, w]_{[1]} .
$$

Similarly, the suspended differential $q_{1}=d_{[1]}=\mathrm{id}_{\llbracket[1]} \otimes d$ is a degree 1 morphism

$$
q_{1}: V[1] \rightarrow V[1], \quad q_{1}\left(v_{[1]}\right)=-(d v)_{[1]} .
$$

Up to the canonical bijective linear map $V \rightarrow V[1], v \mapsto v_{[1]}$, the suspended differential $q_{1}$ and the bilinear operation $q_{2}$ are written simply as

$$
q_{1}(v)=-d v, \quad q_{2}(v \odot w)=(-1)^{\operatorname{deg}_{V}(v)}[v, w],
$$

that is, "the suspended differential is the opposite differential and $q_{2}$ is the twisted Lie bracket".

Define morphisms $q_{k} \in \operatorname{Hom}^{1}\left(\odot^{k}(V[1]), V[1]\right)$ by setting $q_{k} \equiv 0$, for $k \geq 3$. The map

$$
Q^{1}=\sum_{n \geq 1} q_{n}: \bigoplus_{n \geq 1} \bigodot^{n} V[1] \rightarrow V[1]
$$

extends to a coderivation of degree 1

$$
Q: \bigoplus_{n \geq 1} \bigodot^{n} V[1] \rightarrow\left(\bigoplus_{n \geq 1} \bigodot^{n} V[1]\right)
$$

on the reduced symmetric coalgebra cogenerated by $V[1]$, by the formula

$$
\begin{aligned}
& Q\left(v_{1} \odot \cdots \odot v_{n}\right) \\
& \quad=\sum_{k=1}^{n} \sum_{\sigma \in S(k, n-k)} \varepsilon(\sigma) q_{k}\left(v_{\sigma(1)} \odot \cdots \odot v_{\sigma(k)}\right) \odot v_{\sigma(k+1)} \odot \cdots \odot v_{\sigma(n)},
\end{aligned}
$$

where $S(k, n-k)$ is the set of unshuffles and $\varepsilon(\sigma)= \pm 1$ is the Koszul sign, determined by the relation in $\bigodot^{n} V[1]$

$$
v_{\sigma(1)} \odot \cdots \odot v_{\sigma(n)}=\varepsilon(\sigma) v_{1} \odot \cdots \odot v_{n} .
$$

The axioms of differential graded Lie algebra are then equivalent to $Q$ being a codifferential, that is, $Q Q=0$. This description of differential graded Lie algebra in terms of the codifferential $Q$ is called the Quillen construction [1969]. By dropping the requirement that $q_{k} \equiv 0$ for $k \geq 3$ one obtains the notion of $L_{\infty}$-algebra (or strong homotopy Lie algebra); see for example [Lada and Markl 1995; Lada 
and Stasheff 1993; Kontsevich 2003]. Namely, an $L_{\infty}$ structure on a graded vector space $V$ is a sequence of linear maps of degree 1 ,

$$
q_{k}: \bigodot^{k} V[1] \rightarrow V[1], \quad k \geq 1,
$$

such that the induced coderivation $Q$ on the reduced symmetric coalgebra cogenerated by $V[1]$, given by (2-1) is a codifferential, that is, $Q Q=0$. This condition implies $q_{1} q_{1}=0$ and therefore an $L_{\infty}$-algebra is in particular a differential complex. By the preceding discussion, every DGLA can be naturally seen as an $L_{\infty}$-algebra; namely, a DGLA is an $L_{\infty}$-algebra with vanishing higher multiplications $q_{k}, k \geq 3$.

A morphism $f_{\infty}$ between two $L_{\infty}$-algebras

$$
\left(V, q_{1}, q_{2}, q_{3}, \ldots\right) \quad \text { and } \quad\left(W, \hat{q}_{1}, \hat{q}_{2}, \hat{q}_{3}, \ldots\right)
$$

is a sequence of linear maps of degree 0

$$
f_{n}: \bigodot^{n} V[1] \rightarrow W[1], \quad n \geq 1,
$$

such that the morphism of coalgebras

$$
F: \bigoplus_{n \geq 1} \bigodot^{n} V[1] \rightarrow \bigoplus_{n \geq 1} \bigodot^{n} W[1]
$$

induced by $F^{1}=\sum_{n} f_{n}: \bigoplus_{n \geq 1} \bigodot^{n} V[1] \rightarrow W[1]$ commutes with the codifferentials induced by the two $L_{\infty}$ structures on $V$ and $W$ [Fukaya 2003; Kontsevich 2003; Lada and Markl 1995; Lada and Stasheff 1993; Manetti 2004b]. An $L_{\infty^{-}}$ morphism $f_{\infty}$ is called linear (sometimes strict) if $f_{n}=0$ for every $n \geq 2$. Note that a linear map $f_{1}: V[1] \rightarrow W[1]$ is a linear $L_{\infty}$-morphism if and only if

$\hat{q}_{n}\left(f_{1}\left(v_{1}\right) \odot \cdots \odot f_{1}\left(v_{n}\right)\right)=f_{1}\left(q_{n}\left(v_{1} \odot \cdots \odot v_{n}\right)\right)$, for all $n \geq 1, v_{1}, \ldots, v_{n} \in V[1]$.

The category of $L_{\infty}$-algebras will be denoted by $\mathbf{L}_{\infty}$ in this paper. Morphisms between DGLAs are linear morphisms between the corresponding $L_{\infty}$-algebras, so the category of differential graded Lie algebras is a (nonfull) subcategory of $\mathbf{L}_{\infty}$.

If $f_{\infty}$ is an $L_{\infty}$ morphism between $\left(V, q_{1}, q_{2}, q_{3}, \ldots\right)$ and $\left(W, \hat{q}_{1}, \hat{q}_{2}, \hat{q}_{3}, \ldots\right)$, then its linear part $f_{1}: V[1] \rightarrow W[1]$ satisfies the equation $f_{1} \circ q_{1}=\hat{q}_{1} \circ f_{1}$, that is, $f_{1}$ is a map of differential complexes $\left(V[1], q_{1}\right) \rightarrow\left(W[1], \hat{q}_{1}\right)$. An $L_{\infty^{-}}$ morphism $f_{\infty}$ is called a quasiisomorphism of $L_{\infty}$-algebras if its linear part $f_{1}$ is a quasiisomorphism of differential complexes.

A major result in the theory of $L_{\infty}$-algebras is the following homotopical transfer of structure theorem, dating back to Kadeishvili's work on the cohomology of $A_{\infty}$ algebras [Kadeishvili 1982]; see also [Huebschmann and Kadeishvili 1991]. 
Theorem 2.1. Let $\left(V, q_{1}, q_{2}, q_{3}, \ldots\right)$ be an $L_{\infty}$-algebra and $(C, \delta)$ be a differential complex. If there exist two morphisms of differential complexes

$$
\iota:\left(C[1], \delta_{[1]}\right) \rightarrow\left(V[1], q_{1}\right) \quad \text { and } \quad \pi:\left(V[1], q_{1}\right) \rightarrow\left(C[1], \delta_{[1]}\right)
$$

such that the composition $\imath \pi$ is homotopic to the identity, then there exist an $L_{\infty}$ algebra structure $\left(C,\langle\rangle_{1},\langle\rangle_{2}, \ldots\right)$ on $C$ extending its differential complex structure and an $L_{\infty}$-morphism $\imath_{\infty}$ extending $\imath$.

Explicit formulas for the quasiisomorphism $l_{\infty}$ and the brackets \langle\rangle$_{n}$ have been described by Merkulov [1999]; it has then been remarked by Kontsevich and Soibelman [2000; 2001] (see also [Fukaya 2003; Schuhmacher 2004]) that Merkulov's formulas can be nicely written as the summations over rooted trees. Let $K \in \operatorname{Hom}^{-1}(V[1], V[1])$ be an homotopy between $\imath \pi$ and $\operatorname{Id}_{V[1]}$, that is,

$$
q_{1} K+K q_{1}=\imath \pi-\operatorname{Id}_{V[1]},
$$

and denote by $\mathscr{T}_{K, n}$ the groupoid whose objects are directed rooted trees with internal vertices of valence at least two and exactly $n$ tail edges. Trees in $\mathscr{T}_{K, n}$ are decorated as follows: each tail edge of a tree in $\mathscr{T}_{K, n}$ is decorated by the operator $\imath$, each internal edge is decorated by the operator $K$ and also the root edge is decorated by the operator $K$. Every internal vertex $v$ carries the operation $q_{r}$, where $r$ is the number of edges having $v$ as endpoint. Isomorphisms between objects in $\mathcal{T}_{K, n}$ are isomorphisms of the underlying trees. Denote the set of isomorphism classes of objects of $\mathscr{T}_{K, n}$ by the symbol $T_{K, n}$. Similarly, let $\mathscr{T}_{\pi, n}$ be the groupoid whose objects are directed rooted trees with the same decoration as $\mathscr{T}_{K, n}$ except for the root edge, which is decorated by the operator $\pi$ instead of $K$. The set of isomorphism classes of objects of $\mathscr{T}_{\pi, n}$ is denoted $T_{\pi, n}$.

Via the usual operadic rules, each decorated tree $\Gamma \in \mathscr{T}_{K, n}$ gives a linear map

$$
Z_{\Gamma}\left(l, \pi, K, q_{i}\right): C[1]^{\odot n} \rightarrow V[1] .
$$

Similarly, each decorated tree in $\mathscr{T}_{\pi, n}$ gives rise to a degree 1 multilinear operator from $C[1]$ to itself.

Having introduced these notations, we can write Kontsevich-Soibelman's formulas as follows.

Proposition 2.2. In the above set-up the brackets \langle\rangle$_{n}$, and the $L_{\infty}$ morphism $\imath_{\infty}$ can be expressed as sums over decorated rooted trees via the formulas

$$
\iota_{n}=\sum_{\Gamma \in T_{K, n}} \frac{Z_{\Gamma}\left(\imath, \pi, K, q_{i}\right)}{|\operatorname{Aut} \Gamma|}, \quad\langle\rangle_{n}=\sum_{\Gamma \in T_{\pi, n}} \frac{Z_{\Gamma}\left(l, \pi, K, q_{i}\right)}{|\operatorname{Aut} \Gamma|}, \quad n \geq 2 .
$$




\section{The suspended mapping cone of $\chi: L \rightarrow M$.}

The suspended mapping cone of the DGLA morphism $\chi: L \rightarrow M$ is the graded vector space

$$
C_{\chi}=\operatorname{Cone}(\chi)[-1] \text {, }
$$

where $\operatorname{Cone}(\chi)=L[1] \bigoplus M$ is the mapping cone of $\chi$. More explicitly,

$$
C_{\chi}=\bigoplus_{i} C_{\chi}^{i}, \quad C_{\chi}^{i}=L^{i} \bigoplus M^{i-1} .
$$

The suspended mapping cone has a natural differential $\delta \in \operatorname{Hom}^{1}\left(C_{\chi}, C_{\chi}\right)$ given by

$$
\delta(l, m)=(d l, \chi(l)-d m), \quad l \in L, m \in M .
$$

Denote $M[t, d t]=M \otimes \mathbb{K}[t, d t]$ and define, for every $a \in \mathbb{K}$, the evaluation morphism

$$
e_{a}: M[t, d t] \rightarrow M, \quad e_{a}\left(\sum m_{i} t^{i}+n_{i} t^{i} d t\right)=\sum m_{i} a^{i} .
$$

It is easy to prove that every morphism $e_{a}$ is a surjective quasiisomorphism of DGLA. The integral operator $\int_{a}^{b}: \mathbb{K}[t, d t] \rightarrow \mathbb{K}$ extends to a linear map of degree $-1$

$$
\int_{a}^{b}: M[t, d t] \rightarrow M, \quad \int_{a}^{b}\left(\sum_{i} t^{i} m_{i}+t^{i} d t \cdot n_{i}\right)=\sum_{i}\left(\int_{a}^{b} t^{i} d t\right) n_{i} .
$$

Consider the DGLA

$$
H_{\chi}=\left\{(l, m) \in L \times M[t, d t]: e_{0}(m)=0, e_{1}(m)=\chi(l)\right\} .
$$

The morphism

$$
\imath: C_{\chi} \rightarrow H_{\chi}, \quad \imath(l, m)=(l, t \chi(l)+d t \cdot m)
$$

is an injective quasiisomorphism of complexes. If we denote by

$$
\langle\rangle_{1} \in \operatorname{Hom}^{1}\left(C_{\chi}[1], C_{\chi}[1]\right), \quad \text { and } \quad q_{1} \in \operatorname{Hom}^{1}\left(H_{\chi}[1], H_{\chi}[1]\right)
$$

the suspended differentials, namely

$$
\begin{gathered}
\langle(l, m)\rangle_{1}=(-d l,-\chi(l)+d m), \quad l \in L, m \in M, \\
q_{1}(l, m)=(-d l,-d m),
\end{gathered}
$$

then $l$ induces naturally an injective quasiisomorphism

$$
\imath: C_{\chi}[1] \rightarrow H_{\chi}[1], \quad \imath(l, m)=(l, t \chi(l)+d t \cdot m) .
$$

Consider now the linear maps

$$
\pi \in \operatorname{Hom}^{0}\left(H_{\chi}[1], C_{\chi}[1]\right), \quad K \in \operatorname{Hom}^{-1}\left(H_{\chi}[1], H_{\chi}[1]\right)
$$


defined as

$$
\pi(l, m(t, d t))=\left(l, \int_{0}^{1} m(t, d t)\right), \quad K(l, m)=\left(0, \int_{0}^{t} m-t \int_{0}^{1} m\right) .
$$

It is easy to check that $\pi$ is a morphism of complexes and

$$
\pi \imath=\operatorname{Id}_{C_{\chi}[1]}, \quad \imath \pi=\operatorname{Id}_{H_{\chi}[1]}+K q_{1}+q_{1} K .
$$

We are therefore in the hypotheses of Theorem 2.1 and we can transfer the DGLA structure on $H_{\chi}$ to an $L_{\infty}$ structure on $C_{\chi}$. We denote by $\widetilde{C}(\chi)$ the induced $L_{\infty}$ structure on $C_{\chi}$. The universal formulas for the homotopy transfer described in Proposition 2.2 imply that the above construction is functorial. Namely, for every commutative diagram

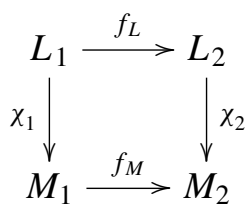

of morphisms of differential graded Lie algebras, the natural map

$$
\left(f_{L}, f_{M}\right): \widetilde{C}\left(\chi_{1}\right) \rightarrow \widetilde{C}\left(\chi_{2}\right)
$$

is a linear $L_{\infty}$-morphism. Summing up:

Theorem 3.1. For any morphism $\chi: L \rightarrow M$ of differential graded Lie algebras, let $\widetilde{C}(\chi)=\left(C_{\chi}, \hat{Q}\right)$ be the $L_{\infty}$-algebra structure defined on $C_{\chi}$ by the above construction. Then

$$
\widetilde{C}: \mathbf{D G L A}^{2} \rightarrow \mathbf{L}_{\infty}
$$

is a functor making the diagram

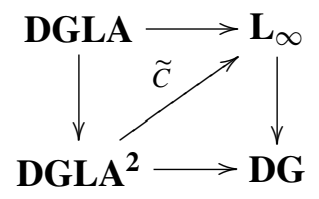

commutative.

Remark 3.2. As an instance of the functoriality, note that the projection on the first factor $p_{1}: \widetilde{C}(\chi) \rightarrow L$ is a linear morphism of $L_{\infty}$-algebras. To see this, consider the morphism in DGLA $^{2}$

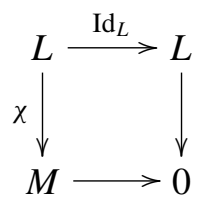


Remark 3.3. The above construction of the $L_{\infty}$ structure on $C_{\chi}$ commutes with the tensor products of differential graded commutative algebras. This means that if $R$ is a DGCA, then the $L_{\infty}$-algebra structure on the suspended mapping cone of $\chi \otimes \mathrm{id}_{\mathrm{R}}: L \otimes R \rightarrow M \otimes R$ is naturally isomorphic to the $L_{\infty}$-algebra $C_{\chi} \otimes R$.

Remark 3.4. The functorial properties of $\widetilde{C}$ determine the $L_{\infty}$ structure $\widetilde{C}(\chi)$ up to (noncanonical) isomorphism. Namley, if $\mathscr{F}: \mathbf{D G L A}^{\mathbf{2}} \rightarrow \mathbf{L}_{\infty}$ is a functor such that the diagram

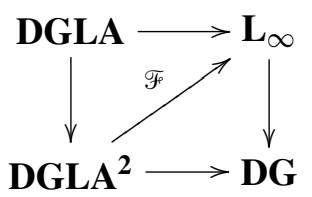

commutes, then for every morphism $\chi$ of differential graded Lie algebras, the $L_{\infty}$ algebra $\mathscr{F}(\chi)$ is isomorphic to $\widetilde{C}(\chi)$. To see this, let

$$
P=\left\{(l, m) \in L \times M[t, d t]: e_{1}(m)=\chi(l)\right\} .
$$

We have a commutative diagram of morphisms of differential graded Lie algebras

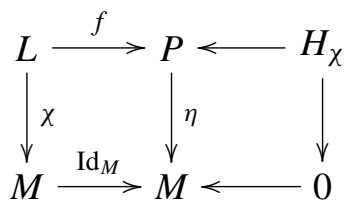

and then two $L_{\infty}$-morphisms $\mathscr{F}(\chi) \rightarrow \mathscr{F}(\eta) \stackrel{h_{\infty}}{\longleftarrow} H_{\chi}$ whose linear parts are the two injective quasiisomorphisms

$$
C_{\chi} \rightarrow C_{\eta} \stackrel{h}{\leftarrow} H_{\chi}, \quad h(l, m)=((l, m), 0) .
$$

A morphism of complexes $p: C_{\eta} \rightarrow H_{\chi}$ such that $p h=\operatorname{Id}_{H_{\chi}}$ can be defined as

$$
p((l, m), n)=\left(l, m+(t-1) e_{0}(m)+d t \cdot n\right) .
$$

The composition of $p$ with the injective quasiisomorphism $C_{\chi} \rightarrow C_{\eta}$ gives the map $\imath$. By general theory of $L_{\infty}$ algebras, there exists a (noncanonical) left inverse of $h_{\infty}$ with linear term equal to $p$. We therefore get an injective $L_{\infty^{-}}$ quasiisomorphism

$$
\hat{\imath}_{\infty}: \mathscr{F}(\chi) \rightarrow H_{\chi}
$$

with linear term $\iota$. The composition of

$$
\iota_{\infty}: \widetilde{C}(\chi) \rightarrow H_{\chi}
$$

with a left inverse of $\hat{\imath}_{\infty}$ is an isomorphism of $L_{\infty}$-algebras between $\widetilde{C}(\chi)$ and $\mathscr{F}(\chi)$. 


\section{The case of semicosimplicial DGLAs}

The $L_{\infty}$ structure on the mapping cone of a DGLA morphism described in Section 3 is actually a particular case of a more general construction of an $L_{\infty}$ structure on the total complex of a semicosimplicial DGLA; see also [Cheng and Getzler 2006], where this construction is described for cosimplicial commutative algebras.

Let $\Delta_{\text {mon }}$ be the category of finite ordinal sets, with order-preserving injective maps between them. A semicosimplicial differential graded Lie algebra is a covariant functor $\Delta_{\text {mon }} \rightarrow$ DGLA. Equivalently, a semicosimplicial DGLA $\mathfrak{g}^{\Delta}$ is a diagram

$$
\mathfrak{g}_{0} \Longrightarrow \mathfrak{g}_{1} \Longrightarrow \mathfrak{g}_{2} \equiv \ldots
$$

where each $\mathfrak{g}_{i}$ is a DGLA, and for each $i>0$ there are $n$ morphisms of DGLAs

$$
\partial_{k, i}: \mathfrak{g}_{i-1} \rightarrow \mathfrak{g}_{i}, \quad k=0, \ldots, i,
$$

such that $\partial_{k+1, i+i} \partial_{l, i}=\partial_{l, i+1} \partial_{k, i}$, for any $k \leq l$. Therefore, the maps

$$
\partial_{i}=\partial_{i, i}-\partial_{i-1, i}+\cdots+(-1)^{i} \partial_{0, i}
$$

endow the vector space $\bigoplus_{i} \mathfrak{g}_{i}$ with the structure of a differential complex. Moreover, being a DGLA, each $\mathfrak{g}_{i}$ is in particular a differential complex

$$
\mathfrak{g}_{i}=\bigoplus_{j} \mathfrak{g}_{i}^{j}, \quad d_{i}: \mathfrak{g}_{i}^{j} \rightarrow \mathfrak{g}_{i}^{j+1},
$$

and since the maps $\partial_{k, i}$ are morphisms of DGLAs, the space

$$
\mathfrak{g}_{\bullet}^{\bullet}=\bigoplus_{i, j} \mathfrak{g}_{i}^{j}
$$

has a natural bicomplex structure. The associated total complex is denoted by $\left(\operatorname{Tot}\left(\mathfrak{g}^{\Delta}\right), \delta\right)$, which has no natural DGLA structure. Yet, it can be endowed with a canonical $L_{\infty}$-algebra structure by homotopy transfer from the homotopy equivalent Thom-Whitney DGLA $\operatorname{Tot}_{T W}\left(\mathfrak{g}^{\Delta}\right)$.

For every $n \geq 0$, denote by $\Omega_{n}$ the differential graded commutative algebra of polynomial differential forms on the standard $n$-simplex $\Delta^{n}$ :

$$
\Omega_{n}=\frac{\mathbb{K}\left[t_{0}, \ldots, t_{n}, d t_{0}, \ldots, d t_{n}\right]}{\left(\sum t_{i}-1, \sum d t_{i}\right)} .
$$

Denote by $\delta^{k, n}: \Omega_{n} \rightarrow \Omega_{n-1}, k=0, \ldots, n$, the face maps then we have natural morphisms of DGLAs

$$
\delta^{k, n}: \Omega_{n} \otimes \mathfrak{g}_{n} \rightarrow \Omega_{n-1} \otimes \mathfrak{g}_{n}, \quad \partial_{k, n}: \Omega_{n-1} \otimes \mathfrak{g}_{n-1} \rightarrow \Omega_{n-1} \otimes \mathfrak{g}_{n}
$$


for every $0 \leq k \leq n$. The Thom-Whitney $D G L A$ is defined as

$$
\operatorname{Tot}_{T W}\left(\mathfrak{g}^{\Delta}\right)=\left\{\left(x_{n}\right)_{n \in \mathbb{N}} \in \bigoplus_{n} \Omega_{n} \otimes \mathfrak{g}_{n} \mid \delta^{k, n} x_{n}=\partial_{k, n} x_{n-1}, \quad \text { for all } 0 \leq k \leq n\right\} .
$$

We denote by $d_{T W}$ the differential of the DGLA $\operatorname{Tot}_{T W}\left(\mathfrak{g}^{\Delta}\right)$. It is a remarkable fact that the integration maps

$$
\int_{\Delta^{n}} \otimes \operatorname{Id}: \Omega_{n} \otimes \mathfrak{g}_{n} \rightarrow \mathbb{K}[n] \otimes \mathfrak{g}_{n}=\mathfrak{g}_{n}[n]
$$

give a quasiisomorphism of differential complexes

$$
I:\left(\operatorname{Tot}_{T W}\left(\mathfrak{g}^{\Delta}\right), d_{T W}\right) \rightarrow\left(\operatorname{Tot}\left(\mathfrak{g}^{\Delta}\right), \delta\right) .
$$

Moreover, Dupont has described in [Dupont 1976; Dupont 1978] an explicit morphism of differential complexes

$$
E: \operatorname{Tot}\left(\mathfrak{g}^{\Delta}\right) \rightarrow \operatorname{Tot}_{T W}\left(\mathfrak{g}^{\Delta}\right)
$$

and an explicit homotopy

$$
h: \operatorname{Tot}_{T W}\left(\mathfrak{g}^{\Delta}\right) \rightarrow \operatorname{Tot}_{T W}\left(\mathfrak{g}^{\Delta}\right)[-1]
$$

such that

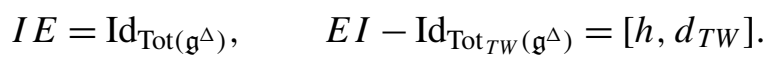

We also refer to the papers [Cheng and Getzler 2006; Getzler 2004; Navarro Aznar 1987] for the explicit description of $E, h$ and for the proof of the above identities. Here we point out that $E$ and $h$ are defined in terms of integration over standard simplexes and multiplication with canonical differential forms and in particular, the construction of $\operatorname{Tot}_{T W}\left(\mathfrak{g}^{\Delta}\right), \operatorname{Tot}\left(\mathfrak{g}^{\Delta}\right), I, E$ and $h$ is functorial in the category DGLA $^{\Delta_{\text {mon }}}$ of semicosimplicial DGLAs.

Therefore we are in the position to use the homotopy transfer of $L_{\infty}$ structures in Theorem 2.1 in order to get a commutative diagram of functors,

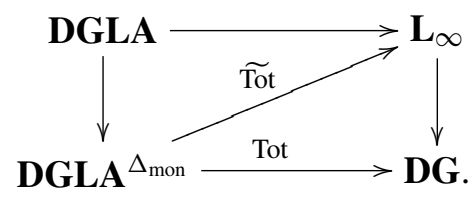

The $L_{\infty}$ structure $\widetilde{C}(\chi)$ on the mapping cone of a DGLA morphism $\chi: L \rightarrow M$ is actually a particular case of this more general construction of the $L_{\infty}$-algebra $\widetilde{\operatorname{Tot}}\left(\mathfrak{g}^{\Delta}\right)$. More precisely, the category DGLA ${ }^{2}$ of morphisms of DGLAs can be seen as a full subcategory of the category of semicosimplicial DGLAs via the 
functor

$$
\{L \stackrel{x}{\rightarrow} M\} \rightsquigarrow\{L \stackrel{0}{\underset{x}{\rightrightarrows}} M \rightleftarrows 0 \underset{\equiv}{\rightrightarrows} \cdots\},
$$

and we have a commutative diagram

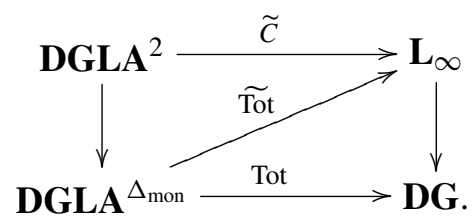

To check the commutativity of this diagram, one only needs to identify the suspended mapping cone $C_{\chi}$ with the total complex $\operatorname{Tot}\left(\chi^{\Delta}\right)$ of the cosimplicial DGLA

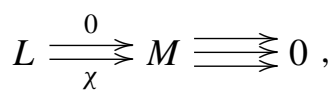

the Thom-Whitney DGLA $\operatorname{Tot}_{T W}\left(\chi^{\Delta}\right)$ with the DGLA we have called $H_{\chi}$ in the main body of the paper, and the Dupont maps $I, E, h$ with the maps we have denoted $\iota, \pi, K$.

For instance, to see that $\operatorname{Tot}\left(\chi^{\Delta}\right) \simeq C_{\chi}$ one only needs to notice that the double complex associated to the cosimplicial DGLA $\chi^{\Delta}$ is

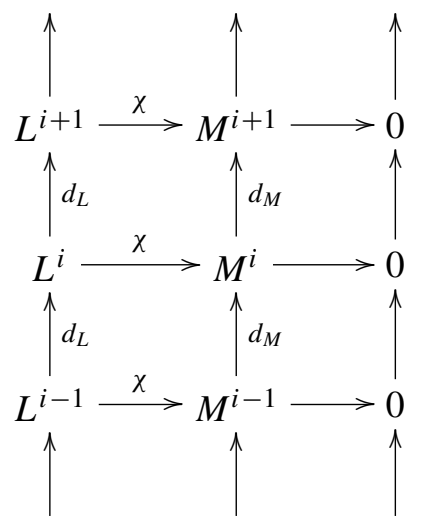

an so the total complex $\operatorname{Tot}\left(\chi^{\Delta}\right)$ is the graded vector space

$$
\operatorname{Tot}\left(\chi^{\Delta}\right)^{i}=L^{i} \bigoplus M^{i-1}
$$

endowed with the total differential

$$
\delta: \operatorname{Tot}\left(\chi^{\Delta}\right)^{i} \rightarrow \operatorname{Tot}\left(\chi^{\Delta}\right)^{i+1}, \quad(l, m) \mapsto\left(d_{L} l, \chi(l)-d_{M} m\right) .
$$


Therefore, the differential complex $\left(\operatorname{Tot}\left(\chi^{\Delta}\right), \delta\right)$ is nothing but the suspended mapping cone $C_{\chi}$ endowed with its usual differential.

Setting $t=t_{0}=1-t_{1}$ we get an identification $\Omega_{1} \simeq \mathbb{K}[t, d t]$ and therefore the Thom-Whitney complex of the semicosimplicial DGLA

$$
L \stackrel{0}{\underset{\chi}{\rightleftarrows}} M M \equiv 0
$$

is isomorphic to the sub-DGLA of $L \bigoplus(\mathbb{K}[t, d t] \otimes M)$ consisting of the differential forms $(l, m(t, d t))$ such that $m(0)=0$ and $m(1)=\chi(l)$, that is, $\operatorname{Tot}_{T W}\left(\chi^{\Delta}\right)=H_{\chi}$.

Moreover, the Dupont maps [Dupont 1976; Navarro Aznar 1987]

$$
\begin{aligned}
E: \operatorname{Tot}\left(\chi^{\Delta}\right) & \rightarrow \operatorname{Tot}_{T W}\left(\chi^{\Delta}\right), \\
(l, m) & \mapsto\left(l, t_{0} \partial_{1,1}(l)-t_{1} \partial_{0,1}(l)-\left(t_{0} d t_{1}-t_{1} d t_{0}\right) m\right)
\end{aligned}
$$

and

$$
\begin{aligned}
I: \operatorname{Tot}_{T W}\left(\chi^{\Delta}\right) & \rightarrow \operatorname{Tot}\left(\chi^{\Delta}\right), \\
\left(l, m\left(t_{0}, t_{1}, d t_{0}, d t_{1}\right)\right) & \mapsto\left(\int_{\Delta^{0}} l, \int_{\Delta^{1}} m\left(t_{0}, t_{1}, d t_{0}, d t_{1}\right)\right)
\end{aligned}
$$

are identified with the maps

$$
\begin{aligned}
& \imath: C_{\chi} \rightarrow H_{\chi}, \\
& (l, m) \mapsto(l, t \chi(l)+d t \cdot m) \\
& \pi: H_{\chi} \rightarrow C_{\chi}, \\
& (l, m(t, d t)) \mapsto\left(l, \int_{0}^{1} m(t, d t)\right) .
\end{aligned}
$$

Finally, we identify the Dupont map $h: \operatorname{Tot}_{T W}\left(\chi^{\Delta}\right) \rightarrow \operatorname{Tot}_{T W}\left(\chi^{\Delta}\right)[-1]$ with the map $K: H_{\chi} \rightarrow H_{\chi}[-1]$. By definition,

$$
\begin{aligned}
h: \operatorname{Tot}_{T W}\left(\chi^{\Delta}\right) & \rightarrow \operatorname{Tot}_{T W}\left(\chi^{\Delta}\right)[-1] \\
\left(l, m\left(t_{0}, t_{1}, d t_{0}, d t_{1}\right)\right) & \mapsto\left(0, t_{0} \cdot h_{0}(m)+t_{1} \cdot h_{1}(m)\right),
\end{aligned}
$$

where $h_{0}$ and $h_{1}$ are the Poincaré homotopies corresponding to the linear contractions of the affine hyperplane $\left\{t_{0}+t_{1}=1\right\} \subseteq \mathbb{A}^{2}$ on the points $(1,0)$ and $(0,1)$ respectively:

$$
h_{i}(m)=\int_{s \in[0,1]} \phi_{i}^{*}(m) \quad \begin{aligned}
\text { with } & \phi_{0}\left(s ; t_{0}, t_{1}\right)=\left((1-s) t_{0}+s,(1-s) t_{1}\right), \\
\text { and } \quad \phi_{1}\left(s ; t_{0}, t_{1}\right) & =\left((1-s) t_{0},(1-s) t_{1}+s\right) .
\end{aligned}
$$

Under the identification $\Omega_{1} \simeq \mathbb{K}[t, d t]$ above, these homotopies read

so

$$
h_{0}(m(t, d t))=\int_{t}^{1} m, \quad h_{1}(m(t, d t))=\int_{t}^{0} m,
$$

$$
t_{0} h_{0}(m)+t_{1} h_{1}(m)=t \int_{t}^{1} m+(1-t) \int_{t}^{0} m=t \int_{0}^{1} m-\int_{0}^{t} m .
$$




\section{A closer look at the $L_{\infty}$ structure on $C_{\chi}$}

We now look for the explicit expressions for the degree 1 linear maps

$$
\langle\rangle_{n}: \bigodot^{n} C_{\chi}[1] \rightarrow C_{\chi}[1], \quad n \geq 2,
$$

defining the $L_{\infty}$ structure $\widetilde{C}(\chi)$, using the Kontsevich-Soibelman formulas described in Proposition 2.2.

The $L_{\infty}$ structure on the differential graded Lie algebra $H_{\chi}$ is given by the brackets

$$
q_{k}: \bigodot^{k}\left(H_{\chi}[1]\right) \rightarrow H_{\chi}[1]
$$

where $q_{k}=0$ for every $k \geq 3$,

$$
q_{1}(l, m(t, d t))=(-d l,-d m(t, d t))
$$

and

$$
\begin{aligned}
q_{2}\left(\left(l_{1}, m_{1}(t, d t)\right) \odot\right. & \left.\left(l_{2}, m_{2}(t, d t)\right)\right) \\
& =(-1)^{\operatorname{deg}_{H_{\chi}}\left(l_{1}, m_{1}(t, d t)\right)}\left(\left[l_{1}, l_{2}\right],\left[m_{1}(t, d t), m_{2}(t, d t)\right]\right) .
\end{aligned}
$$

The properties

$$
q_{2}(\operatorname{Im} K \otimes \operatorname{Im} K) \subseteq \operatorname{ker} \pi \cap \operatorname{ker} K, \quad q_{k}=0 \text { for all } k \geq 3,
$$

imply that, fixing the number $n \geq 2$ of tails, there exists at most one isomorphism class of rooted trees giving a nontrivial contribution to \langle\rangle$_{n}$.

- $n=2$ :

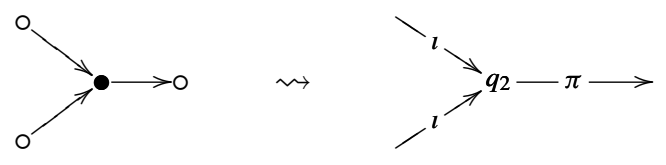

This graph gives

$$
\left\langle\gamma_{1} \odot \gamma_{2}\right\rangle_{2}=\pi q_{2}\left(l\left(\gamma_{1}\right) \odot l\left(\gamma_{2}\right)\right) .
$$

- $n \geq 3$ :
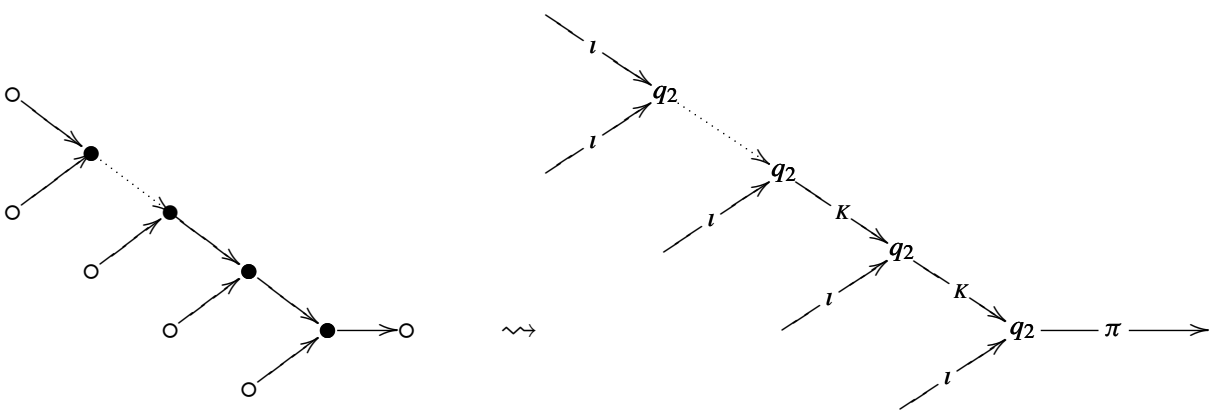
This graph gives, for every $n \geq 3$, the formula

$$
\begin{aligned}
& \left\langle\gamma_{1} \odot \cdots \odot \gamma_{n}\right\rangle_{n}= \\
& =\frac{1}{2} \sum_{\sigma \in S_{n}} \varepsilon(\sigma) \pi q_{2}\left(l\left(\gamma_{\sigma(1)}\right) \odot K q_{2}\left(l\left(\gamma_{\sigma(2)}\right) \odot \cdots \odot K q_{2}\left(l\left(\gamma_{\sigma(n-1)}\right) \odot \imath\left(\gamma_{\sigma(n)}\right)\right) \cdots\right)\right) \\
& =\sum_{\substack{\sigma \in S_{n} \\
\sigma(n-1)<\sigma(n)}} \varepsilon(\sigma) \pi q_{2}\left(l\left(\gamma_{\sigma(1)}\right) \odot K q_{2}\left(l\left(\gamma_{\sigma(2)}\right) \odot \cdots \odot K q_{2}\left(l\left(\gamma_{\sigma(n-1)}\right) \odot \imath\left(\gamma_{\sigma(n)}\right)\right) \cdots\right)\right) .
\end{aligned}
$$

A more refined description involving the original brackets in the differential graded Lie algebras $L$ and $M$ is obtained by decomposing the symmetric powers of $C_{\chi}[1]$ into types

$$
\bigodot^{n}\left(C_{\chi}[1]\right)=\bigodot^{n} \operatorname{Cone}(\chi)=\bigoplus_{\lambda+\mu=n}\left(\bigodot^{\mu} M\right) \otimes\left(\bigodot^{\lambda} L[1]\right)
$$

The operation \langle\rangle$_{2}$ decomposes into

$$
\begin{aligned}
& l_{1} \odot l_{2} \mapsto(-1)^{\operatorname{deg}_{L}\left(l_{1}\right)}\left[l_{1}, l_{2}\right] \in L, \quad m_{1} \odot m_{2} \mapsto 0, \\
& m \otimes l \mapsto \frac{(-1)^{\operatorname{deg}_{M}(m)+1}}{2}[m, \chi(l)] \in M .
\end{aligned}
$$

For every $n \geq 2$, it is easy to see that $\left\langle\gamma_{1} \odot \cdots \odot \gamma_{n+1}\right\rangle_{n+1}$ can be nonzero only if the multivector $\gamma_{1} \odot \cdots \odot \gamma_{n+1}$ belongs to $\bigodot^{n} M \otimes L[1]$. For $n \geq 2$, $m_{1}, \ldots, m_{n} \in M$, and $l \in L[1]$, the formula for \langle\rangle$_{n+1}$ described above becomes

$$
\begin{aligned}
& \left\langle m_{1} \odot \cdots \odot m_{n} \otimes l\right\rangle_{n+1}= \\
& \sum_{\sigma \in S_{n}} \varepsilon(\sigma) \pi q_{2}\left((d t) m_{\sigma(1)} \odot K q_{2}\left((d t) m_{\sigma(2)} \odot \cdots \odot K q_{2}\left((d t) m_{\sigma(n)} \otimes t \chi(l)\right) \cdots\right)\right) .
\end{aligned}
$$

Define recursively a sequence of polynomials $\phi_{i}(t) \in \mathbb{Q}[t] \subseteq \mathbb{K}[t]$ and rational numbers $I_{n}$ by the rule

$$
\phi_{1}(t)=t, \quad I_{n}=\int_{0}^{1} \phi_{n}(t) d t, \quad \phi_{n+1}(t)=\int_{0}^{t} \phi_{n}(s) d s-t I_{n} .
$$

By the definition of the homotopy operator $K$ we have, for every $m \in M$,

$$
K\left(\left(\phi_{n}(t) d t\right) m\right)=\phi_{n+1}(t) m .
$$

Therefore, for every $m_{1}, m_{2} \in M$ we have

$$
K q_{2}\left(\left(d t \cdot m_{1}\right) \odot \phi_{n}(t) m_{2}\right)=-(-1)^{\operatorname{deg}_{M}\left(m_{1}\right)} \phi_{n+1}(t)\left[m_{1}, m_{2}\right] .
$$


Therefore, we find

$$
\begin{aligned}
& \left\langle m_{1} \odot \cdots \odot m_{n} \otimes l\right\rangle_{n+1} \\
& =\sum_{\sigma \in S_{n}} \varepsilon(\sigma) \pi q_{2}\left(d t m_{\sigma(1)} \odot K q_{2}\left(d t m_{\sigma(2)} \odot \cdots \odot K q_{2}\left(d t m_{\sigma(n)} \otimes t \chi(l)\right) \cdots\right)\right) \\
& =(-1)^{1+\operatorname{deg}_{M}\left(m_{\sigma(n)}\right)} \sum_{\sigma \in S_{n}} \varepsilon(\sigma) \pi q_{2}\left(d t m_{\sigma(1)} \quad \odot K q_{2}\left(d t m_{\sigma(2)} \odot \cdots \odot \phi_{2}(t)\left[m_{\sigma(n)}, \chi(l)\right] \cdots\right)\right) \\
& =(-1)^{n-1+\sum_{i=2}^{n} \operatorname{deg}_{M}\left(m_{\sigma(i)}\right)} \sum_{\sigma \in S_{n}} \varepsilon(\sigma) \pi q_{2}\left(d t m _ { \sigma ( 1 ) } \odot \phi _ { n } ( t ) \left[m_{\sigma(2)}, \ldots,\right.\right. \\
& \left.\left.\quad\left[m_{\sigma(n)}, \chi(l)\right] \cdots\right]\right) \\
& =(-1)^{n+\sum_{i=1}^{n} \operatorname{deg}_{M}\left(m_{i}\right)} I_{n} \sum_{\sigma \in S_{n}} \varepsilon(\sigma)\left[m_{\sigma(1)},\left[m_{\sigma(2)}, \ldots,\left[m_{\sigma(n)}, \chi(l)\right] \cdots\right]\right],
\end{aligned}
$$

which lies in $M$.

We also have an explicit expression for the coefficients $I_{n}$ appearing in the formula for \langle\rangle$_{n+1}$; in the next lemma we show that they are, up to a sign, the Bernoulli numbers.

Lemma 5.1. For every $n \geq 1$ we have $I_{n}=-B_{n} / n$ !, where $B_{n}$ are the Bernoulli numbers, that is, the rational numbers defined by the series expansion identity

$$
\sum_{n=0}^{\infty} B_{n} \frac{x^{n}}{n !}=\frac{x}{e^{x}-1}=1-\frac{x}{2}+\frac{x^{2}}{12}-\frac{x^{4}}{720}+\frac{x^{6}}{30240}-\frac{x^{8}}{1209600}+\cdots
$$

Proof. Keeping in mind the definition of $B_{n}$, we have to prove that

$$
1-\sum_{n=1} I_{n} x^{n}=\frac{x}{e^{x}-1} .
$$

Consider the polynomials $\psi_{0}(t)=1$ and $\psi_{n}(t)=\phi_{n}(t)-I_{n}$ for $n \geq 1$. Then, for any $n \geq 1$,

$$
\frac{d}{d t} \psi_{n}(t)=\psi_{n-1}(t), \quad \int_{0}^{1} \psi_{n}(t) d t=0
$$

Setting

$$
F(t, x)=\sum_{n=0}^{\infty} \psi_{n}(t) x^{n}
$$

we have

$$
\frac{d}{d t} F(t, x)=\sum_{n=1}^{\infty} \psi_{n-1}(t) x^{n}=x F(t, x), \quad \int_{0}^{1} F(t, x) d t=1 .
$$


Therefore, $F(t, x)=F(0, x) e^{t x}$,

$$
1=\int_{0}^{1} F(t, x) d t=F(0, x) \int_{0}^{1} e^{t x} d t=F(0, x) \frac{e^{x}-1}{x},
$$

and then

$$
F(0, x)=\frac{x}{e^{x}-1} .
$$

Since $\psi_{n}(0)=-I_{n}$ for any $n \geq 1$ we get

$$
\frac{x}{e^{x}-1}=F(0, x)=1-\sum_{n=1}^{\infty} I_{n} x^{n} .
$$

In fact an alternative proof of the equality $I_{n}=-B_{n} / n$ ! can be done by observing that the polynomials $n ! \psi_{n}(t)$ satisfy the recursive relations of the Bernoulli polynomials; see for example [Remmert 1991].

Summing up the results of this section, we have the following explicit description of the $L_{\infty}$ algebra $\widetilde{C}(\chi)$.

Theorem 5.2. The $L_{\infty}$ algebra $\widetilde{C}(\chi)$ is defined by the multilinear maps

$$
\langle\rangle_{n}: \bigodot^{n} C_{\chi}[1] \rightarrow C_{\chi}[1]
$$

given by

$$
\begin{array}{ll}
\langle(l, m)\rangle_{1}=(-d l,-\chi(l)+d m), & \left\langle l_{1} \odot l_{2}\right\rangle_{2}=(-1)^{\operatorname{deg}_{L}\left(l_{1}\right)}\left[l_{1}, l_{2}\right], \\
\left\langle m_{1} \odot m_{2}\right\rangle_{2}=0, & \langle m \otimes l\rangle_{2}=\frac{1}{2}(-1)^{\operatorname{deg}_{M}(m)+1}[m, \chi(l)], \\
\left\langle m_{1} \odot \cdots \odot m_{n} \otimes l_{1} \odot \cdots \odot l_{k}\right\rangle_{n+k}=0 & \text { if } n+k \geq 3 \text { and } k \neq 1,
\end{array}
$$

and

$$
\begin{aligned}
\left\langle m_{1} \odot\right. & \left.\cdots \odot m_{n} \otimes l\right\rangle_{n+1} \\
& =-(-1)^{\sum_{i=1}^{n} \operatorname{deg}_{M}\left(m_{i}\right)} \frac{B_{n}}{n !} \sum_{\sigma \in S_{n}} \varepsilon(\sigma)\left[m_{\sigma(1)},\left[m_{\sigma(2)}, \ldots,\left[m_{\sigma(n)}, \chi(l)\right] \cdots\right]\right]
\end{aligned}
$$

if $n \geq 2$. Here the $B_{n}$ are the Bernoulli numbers, that is, the rational numbers defined by the series expansion identity

$$
\sum_{n=0}^{\infty} B_{n} \frac{x^{n}}{n !}=\frac{x}{e^{x}-1}=1-\frac{x}{2}+\frac{x^{2}}{12}-\frac{x^{4}}{720}+\frac{x^{6}}{30240}-\frac{x^{8}}{1209600}+\cdots
$$

Remark 5.3. Via the decalage isomorphism $\bigodot^{n}\left(C_{\chi}[1]\right) \stackrel{\sim}{\rightarrow}\left(\bigwedge^{n} C_{\chi}\right)[n]$, the linear maps \langle\rangle$_{n}$ defining the $L_{\infty}$-algebra $\widetilde{C}(\chi)$ correspond to multilinear operations [ $]_{n}$ : 
$\bigwedge^{n} C_{\chi} \rightarrow C_{\chi}[2-n]$ on $C_{\chi}$. In particular, the linear map \langle\rangle$_{1}$ corresponds to the differential $\delta$ on $C_{\chi}$,

$$
\delta:(l, m) \mapsto(d l, \chi(l)-d m),
$$

whereas the map \langle\rangle$_{2}$ corresponds to the degree-zero bracket

$$
[]_{2}: C_{\chi} \wedge C_{\chi} \rightarrow C_{\chi}
$$

given by

$$
\left[l_{1}, l_{2}\right]_{2}=\left[l_{1}, l_{2}\right], \quad[m, l]_{2}=\frac{1}{2}[m, \chi(l)], \quad\left[m_{1}, m_{2}\right]_{2}=0 .
$$

This is precisely the naive bracket described in the Introduction.

Remark 5.4. The occurrence of Bernoulli numbers is not surprising. It had already been noticed by K. T. Chen [1957] how Bernoulli numbers are related to the coefficients of the Baker-Campbell-Hausdorff formula.

More recently, the relevance of Bernoulli numbers in deformation theory has been also remarked by Ziv Ran [2004]. In particular, Ran's "JacoBer" complex provides an independent description of the $L_{\infty}$ structure $\widetilde{C}(\chi)$; see also [Merkulov 2005].

Bernoulli numbers also appear in some expressions of the gauge equivalence in a differential graded Lie algebra [Sullivan 2007; Getzler 2004]. In fact the relation $x=e^{a} * y$ can be written as

$$
x-y=\frac{e^{\mathrm{ad}_{a}}-1}{\operatorname{ad}_{a}}([a, y]-d a) .
$$

Applying to both sides the inverse of the operator $\left(e^{\operatorname{ad}_{a}}-1\right) / \operatorname{ad}_{a}$ we get

$$
d a=[a, y]-\sum_{n \geq 0} \frac{B_{n}}{n !} \operatorname{ad}_{a}^{n}(x-y) .
$$

The multilinear brackets \langle\rangle$_{n}$ on Cone $(\chi)=C_{\chi}[1]$ can be related to the Koszul (or "higher derived") brackets $\Phi_{n}$ of a differential graded Lie algebra as follows. Let $(M, \partial,[]$,$) be a differential graded Lie algebra. The Koszul brackets$

$$
\Phi_{n}: \bigodot^{n} M \rightarrow M, \quad n \geq 1
$$

are the degree-1 linear maps defined as $\Phi_{1}=0$ and

$$
\Phi_{n}\left(m_{1} \cdots m_{n}\right)=\frac{1}{n !} \sum_{\sigma \in \Sigma_{n}} \varepsilon(\sigma)\left[\cdot\left[\left[\partial m_{\sigma(1)}, m_{\sigma(2)}\right], m_{\sigma(3)}\right], \ldots, m_{\sigma(n)}\right]
$$

for $n \geq 2$. Let $L$ be the differential graded Lie subalgebra of $M$, given by $\partial M$ and let $\chi: L \rightarrow M$ be the inclusion. We can identify $M$ with the image of the injective 
linear map $M \hookrightarrow$ Cone $(\chi)$ given by $m \mapsto(\partial m, m)$. Then we have $\langle(\partial m, m)\rangle_{1}=0$,

$$
\left\langle\left(\partial m_{1}, m_{1}\right) \odot\left(\partial m_{2}, m_{2}\right)\right\rangle_{2}=\left(\partial \Phi_{2}\left(m_{1}, m_{2}\right), \Phi_{2}\left(m_{1}, m_{2}\right)\right)
$$

and, for $n \geq 2$,

$$
\begin{aligned}
& \left\langle\left(\partial m_{1}, m_{1}\right) \odot \cdots \odot\left(\partial m_{n+1}, m_{n+1}\right)\right\rangle_{n+1} \\
& \quad=\left(0, B_{n}(-1)^{n}(n+1) \Phi_{n+1}\left(m_{1} \odot \cdots \odot m_{n+1}\right)\right) .
\end{aligned}
$$

Since the multilinear operations \langle\rangle$_{n}$ define an $L_{\infty}$-algebra structure on $C_{\chi}=$ Cone $(\chi)[-1]$, they satisfy a sequence of quadratic relations. Due to the already mentioned correspondence with the Koszul brackets, these relations are translated into a sequence of differential or quadratic relations between the odd Koszul brackets, defined as $\{m\}_{1}=0$ and

$$
\left\{m_{1}, \ldots, m_{n}\right\}_{n}=\frac{1}{n !} \sum_{\sigma \in \Sigma_{n}} \varepsilon(\sigma)(-1)^{\sigma}\left[\cdot\left[\left[\partial m_{\sigma(1)}, m_{\sigma(2)}\right], m_{\sigma(3)}\right], \ldots, m_{\sigma(n)}\right]
$$

for $n \geq 2$. For instance, if $m_{1}, m_{2}, m_{3}$ are homogeneous elements of degree $i_{1}, i_{2}, i_{3}$ respectively, then

$$
\begin{aligned}
\left\{\left\{m_{1}, m_{2}\right\}_{2}, m_{3}\right\}+(-1)^{i_{1} i_{2}+i_{1} i_{3}} & \left\{\left\{m_{2}, m_{3}\right\}_{2}, m_{1}\right\}_{2} \\
+ & (-1)^{i_{2} i_{3}+i_{1} i_{3}}\left\{\left\{m_{3}, m_{1}\right\}_{2}, m_{2}\right\}_{2}=\frac{3}{2} \partial\left\{m_{1}, m_{2}, m_{3}\right\}_{3} .
\end{aligned}
$$

The occurrence of Bernoulli numbers in the $L_{\infty}$-type structure defined by the higher Koszul brackets has been recently remarked by K. Bering [2006].

\section{The Maurer-Cartan functor}

Having introduced an $L_{\infty}$ structure on $C_{\chi}$ in Section 5, we have a corresponding Maurer-Cartan functor [Fukaya 2003; Kontsevich 2003] $\mathrm{MC}_{C_{\chi}}:$ Art $\rightarrow$ Set, defined as

$$
\operatorname{MC}_{C_{\chi}}(A)=\left\{\gamma \in C_{\chi}[1]^{0} \otimes \mathfrak{m}_{A}: \sum_{n \geq 1} \frac{\left\langle\gamma^{\odot n}\right\rangle_{n}}{n !}=0\right\}, \quad A \in \text { Art. }
$$

With $\gamma=(l, m), l \in L^{1} \otimes \mathfrak{m}_{A}$ and $m \in M^{0} \otimes \mathfrak{m}_{A}$, the Maurer-Cartan equation becomes

$$
0=\sum_{n=1}^{\infty} \frac{\left\langle(l, m)^{\odot n}\right\rangle_{n}}{n !}
$$




$$
\begin{aligned}
& =\langle(l, m)\rangle_{1}+\frac{1}{2}\left\langle l^{\odot 2}\right\rangle_{2}+\langle m \otimes l\rangle_{2}+\frac{1}{2}\left\langle m^{\odot 2}\right\rangle_{2}+\sum_{n \geq 2} \frac{n+1}{(n+1) !}\left\langle m^{\odot n} \otimes l\right\rangle_{n+1} \\
& =-d l-\frac{1}{2}[l, l],-\chi(l)+d m-\frac{1}{2}[m, \chi(l)]+\sum_{n \geq 2} \frac{1}{n !}\left\langle m^{\odot n} \otimes l\right\rangle_{n+1},
\end{aligned}
$$

which lies in $\left(L^{2} \oplus M^{1}\right) \otimes \mathfrak{m}_{A}$.

According to Theorem 5.2, since $\operatorname{deg}_{M}(m)=\operatorname{deg}_{C_{\chi[1]}}(m)=0$, we have

$$
\left\langle m^{\odot n} \otimes l\right\rangle_{n+1}=-\frac{B_{n}}{n !} \sum_{\sigma \in S_{n}}[m,[m, \ldots,[m, \chi(l)] \cdots]]=-B_{n} \operatorname{ad}_{m}^{n}(\chi(l)),
$$

where for $a \in M^{0} \otimes \mathfrak{m}_{A}$ we denote by $\operatorname{ad}_{a}: M \otimes \mathfrak{m}_{A} \rightarrow M \otimes \mathfrak{m}_{A}$ the operator $\operatorname{ad}_{a}(y)=[a, y]$.

The Maurer-Cartan equation on $C_{\chi}$ is therefore equivalent to

$$
\left\{\begin{array}{l}
d l+\frac{1}{2}[l, l]=0, \\
\chi(l)-d m+\frac{1}{2}[m, \chi(l)]+\sum_{n=2}^{\infty} \frac{B_{n}}{n !} \operatorname{ad}_{m}^{n}(\chi(l))=0 .
\end{array}\right.
$$

Since $B_{0}=1$ and $B_{1}=-\frac{1}{2}$, we can write the second equation as

$$
\begin{aligned}
0 & =\chi(l)-d m+\frac{1}{2}[m, \chi(l)]+\sum_{n=2}^{\infty} \frac{B_{n}}{n !} \operatorname{ad}_{m}^{n}(\chi(l)) \\
& =[m, \chi(l)]-d m+\sum_{n=0}^{\infty} \frac{B_{n}}{n !} \operatorname{ad}_{m}^{n}(\chi(l))=[m, \chi(l)]-d m+\frac{\operatorname{ad}_{m}}{e^{\operatorname{ad}_{m}}-1}(\chi(l)) .
\end{aligned}
$$

Applying the invertible operator $\left(e^{\mathrm{ad}_{m}}-1\right) / \mathrm{ad}_{m}$ we get

$$
0=\chi(l)+\frac{e^{\mathrm{ad}_{m}}-1}{\operatorname{ad}_{m}}([m, \chi(l)]-d m) .
$$

On the right-hand side of the last formula we recognize the explicit description of the gauge action

$$
\begin{gathered}
\exp \left(M^{0} \otimes \mathfrak{m}_{A}\right) \times M^{1} \otimes \mathfrak{m}_{A} \stackrel{*}{\longrightarrow} M^{1} \otimes \mathfrak{m}_{A}, \\
e^{a} * y=y+\sum_{n=0}^{+\infty} \frac{\operatorname{ad}_{a}^{n}}{(n+1) !}([a, y]-d a)=y+\frac{e^{\operatorname{ad}_{a}}-1}{\operatorname{ad}_{a}}([a, y]-d a) .
\end{gathered}
$$

Therefore, the Maurer-Cartan equation for the $L_{\infty}$-algebra structure on $C_{\chi}$ is equivalent to

$$
\left\{\begin{array}{l}
d l+\frac{1}{2}[l, l]=0 \\
e^{m} * \chi(l)=0
\end{array}\right.
$$




\section{Homotopy equivalence and the deformation functor}

Recall that the deformation functor associated to an $L_{\infty}$-algebra $\mathfrak{g}$ is

$$
\operatorname{Def}_{\mathfrak{g}}=\mathrm{MC}_{\mathfrak{g}} / \sim
$$

where $\sim$ denotes homotopy equivalence of solutions of the Maurer-Cartan equation: two elements $\gamma_{0}$ and $\gamma_{1}$ of $\mathrm{MC}_{\mathfrak{g}}(A)$ are called homotopy equivalent if there exists an element $\gamma(t, d t) \in \operatorname{MC}_{\mathfrak{g}[t, d t]}(A)$ with $\gamma(0)=\gamma_{0}$ and $\gamma(1)=\gamma_{1}$.

Remark 7.1. The homotopy equivalence is an equivalence relation and a proof of this fact can be found in [Manetti 2004b, Ch. 9]. The same conclusion also follows immediately from the more general result [Getzler 2004, Prop. 4.7] that the simplicial set $\left\{\mathrm{MC}_{\mathfrak{g} \otimes \Omega_{n}}(A)\right\}_{n \in \mathbb{N}}$ is a Kan complex, where $\Omega_{n}$ is the DG commutative algebra of polynomial differential forms on the standard $n$-simplex.

We have already described the functor $\mathrm{MC}_{C_{\chi}}$ in terms of the Maurer-Cartan equation in $L$ and the gauge action in $M$. Now we want to prove a similar result for the homotopy equivalence on $\mathrm{MC}_{C_{\chi}}$. We need some preliminary results.

Proposition 7.2. Let $(L, d,[]$,$) be a differential graded Lie algebra such that$

(1) $L=M \bigoplus C \bigoplus D$ as graded vector spaces,

(2) $M$ is a differential graded subalgebra of $L$, and

(3) $d: C \rightarrow D[1]$ is an isomorphism of graded vector spaces.

Then, for every $A \in$ Art there exists a bijection

$$
\alpha: \operatorname{MC}_{M}(A) \times\left(C^{0} \otimes \mathfrak{m}_{A}\right) \stackrel{\sim}{\longrightarrow} \operatorname{MC}_{L}(A), \quad(x, c) \mapsto e^{c} * x .
$$

Proof. This is essentially proved in [Schlessinger and Stasheff 1979, Section 5] by the induction of the length of $A$ and using the Baker-Campbell-Hausdorff formula. Here we sketch a different proof based on formal theory of deformation functors [Schlessinger 1968; Rim 1972; Fantechi and Manetti 1998; Manetti 1999].

The map $\alpha$ is a natural transformation of homogeneous functors, so it is sufficient to show that $\alpha$ is bijective on tangent spaces and injective on obstruction spaces. Recall that the tangent space of $\mathrm{MC}_{L}$ is $Z^{1}(L)$, while its obstruction space is $H^{2}(L)$. The functor $A \mapsto C^{0} \otimes \mathfrak{m}_{A}$ is smooth with tangent space $C^{0}$ and therefore tangent and obstruction spaces of the functor

$$
A \mapsto \mathrm{MC}_{M}(A) \times\left(C^{0} \otimes \mathfrak{m}_{A}\right)
$$

are respectively $Z^{1}(M) \oplus C^{0}$ and $H^{2}(M)$. The tangent map is

$$
\begin{aligned}
Z^{1}(M) \bigoplus C^{0} \ni(x, c) & \mapsto \\
e^{c} * x & =x-d c \in Z^{1}(M) \bigoplus d\left(C^{0}\right)=Z^{1}(M) \bigoplus D^{1}=Z^{1}(L)
\end{aligned}
$$


and it is an isomorphism. The inclusion $M \hookrightarrow L$ is a quasiisomorphism, therefore the obstruction to lifting $x$ in $M$ is equal to the obstruction to lifting $x=e^{0} * x$ in $L$. We conclude the proof by observing that, according to [Fantechi and Manetti 1998, Prop. 7.5], [Manetti 1999, Lemma 2.20], the obstruction maps of Maurer-Cartan functor are invariant under the gauge action.

Corollary 7.3. Let $M$ be a differential graded Lie algebra, $L=M[t, d t]$ and $C \subseteq M[t]$ the subspace consisting of polynomials $g(t)$ with $g(0)=0$. Then for every $A \in$ Art the map $(x, g(t)) \mapsto e^{g(t)} * x$ induces an isomorphism

$$
\operatorname{MC}_{M}(A) \times\left(C^{0} \otimes \mathfrak{m}_{A}\right) \simeq \operatorname{MC}_{L}(A) .
$$

Proof. The data $M, C$ and $D=d(C)$ satisfy the condition of Proposition 7.2.

Corollary 7.4. Let $M$ be a differential graded Lie algebra. Two elements $x_{0}, x_{1} \in$ $\mathrm{MC}_{M}(A)$ are gauge equivalent if and only if they are homotopy equivalent.

Proof. If $x_{0}$ and $x_{1}$ are gauge equivalent, then there exists $g \in M^{0} \otimes \mathfrak{m}_{A}$ such that $e^{g} * x_{0}=x_{1}$. Then, by Corollary 7.3. $x(t)=e^{t g} * x_{0}$ is an element of $\operatorname{MC}_{M[t, d t]}(A)$ with $x(0)=x_{0}$ and $x(1)=x_{1}$, that is, $x_{0}$ and $x_{1}$ are homotopy equivalent.

Vice versa, if $x_{0}$ and $x_{1}$ are homotopy equivalent, there exists

$$
x(t) \in \mathrm{MC}_{M[t, d t]}(A)
$$

such that $x(0)=x_{0}$ and $x(1)=x_{1}$. By Corollary 7.3., there exists $g(t) \in M^{0}[t] \otimes \mathfrak{m}_{A}$ with $g(0)=0$ such that $x(t)=e^{g(t)} * x_{0}$. Then $x_{1}=e^{g(1)} * x_{0}$, that is, $x_{0}$ and $x_{1}$ are gauge equivalent.

Theorem 7.5. Let $\chi: L \rightarrow M$ be a morphism of differential graded Lie algebras and let $\left(l_{0}, m_{0}\right)$ and $\left(l_{1}, m_{1}\right)$ be elements of $\mathrm{MC}_{C_{\chi}}(A)$. Then $\left(l_{0}, m_{0}\right)$ is homotopically equivalent to $\left(l_{1}, m_{1}\right)$ if and only if there exists $(a, b) \in C_{\chi}^{0} \otimes \mathfrak{m}_{A}$ such that

$$
l_{1}=e^{a} * l_{0}, \quad e^{m_{1}}=e^{d b} e^{m_{0}} e^{-\chi(a)} .
$$

Remark 7.6. The condition $e^{m_{1}}=e^{d b} e^{m_{0}} e^{-\chi(a)}$ can be also written as $m_{1} \bullet \chi(a)=$ $d b \bullet m_{0}$, where $\bullet$ is the Baker-Campbell-Hausdorff product in the nilpotent Lie algebra $M^{0} \otimes \mathfrak{m}_{A}$.

As a consequence, we get that in this case the homotopy equivalence is induced by a group action, which is false for general $L_{\infty}$-algebras.

Proof. We shall say that two elements $\left(l_{0}, m_{0}\right),\left(l_{1}, m_{1}\right)$ are gauge equivalent if and only if there exists $(a, b) \in C_{\chi}^{0} \otimes \mathfrak{m}_{A}$ such that

$$
l_{1}=e^{a} * l_{0}, \quad e^{m_{1}}=e^{d b} e^{m_{0}} e^{-\chi(a)} .
$$

We first show that homotopy implies gauge. Let $\left(l_{0}, m_{0}\right)$ and $\left(l_{1}, m_{1}\right)$ be homotopy equivalent elements of $\operatorname{MC}_{C_{\chi}}(A)$. Then there exists an element $(\tilde{l}, \tilde{m})$ of 
$\operatorname{MC}_{C_{\chi}[s, d s]}(A)$ with $(\tilde{l}(0), \tilde{m}(0))=\left(l_{0}, m_{0}\right)$ and $(\tilde{l}(1), \tilde{m}(1))=\left(l_{1}, m_{1}\right)$. According to Remark 3.3, the Maurer-Cartan equation for $(\tilde{l}, \tilde{m})$ is

$$
\left\{\begin{array}{l}
d \tilde{l}+\frac{1}{2}[\tilde{l}, \tilde{l}]=0 \\
e^{\tilde{m}} * \chi(\tilde{l})=0
\end{array}\right.
$$

The first of the two equations above tells us that $\tilde{l}$ is a solution of the Maurer-Cartan equation for $L[s, d s]$. So, by Corollary 7.3, there exists a degree zero element $\lambda(s)$ in $L[s] \otimes \mathfrak{m}_{A}$ with $\lambda(0)=0$ such that $\tilde{l}=e^{\lambda} * l_{0}$. Evaluating at $s=1$ we find $l_{1}=e^{\lambda_{1}} * l_{0}$. As a consequence of $\tilde{l}=e^{\lambda} * l_{0}$, we also have $\chi(\tilde{l})=e^{\chi(\lambda)} * \chi\left(l_{0}\right)$. Set $\tilde{\mu}=\tilde{m} \bullet \chi(\lambda) \bullet\left(-m_{0}\right)$, so that $\tilde{m}=\tilde{\mu} \bullet m_{0} \bullet(-\chi(\lambda))$ and the second Maurer-Cartan equation is reduced to $e^{\tilde{\mu}} *\left(e^{m_{0}} * \chi\left(l_{0}\right)\right)=0$, that is, to $e^{\tilde{\mu}} * 0=0$, where we have used the fact that $\left(l_{0}, m_{0}\right)$ is a solution of the Maurer-Cartan equation in $C_{\chi}$. This last equation is equivalent to the equation $d \tilde{\mu}=0$ in $C_{\chi}[s, d s] \otimes \mathfrak{m}_{A}$. If we write $\tilde{\mu}(s, d s)=\mu^{0}(s)+d s \mu^{-1}(s)$, then the equation $d \tilde{\mu}=0$ becomes

$$
\left\{\begin{array}{l}
\dot{\mu}^{0}-d_{M} \mu^{-1}=0 \\
d_{M} \mu^{0}=0
\end{array}\right.
$$

where $d_{M}$ is the differential in the DGLA $M$. The solution is, for any fixed $\mu^{-1}$,

$$
\mu^{0}(s)=\int_{0}^{s} d \sigma d_{M} \mu^{-1}(\sigma)=-d_{M} \int_{0}^{s} d \sigma \mu^{-1}(\sigma) .
$$

Set $v=-\int_{0}^{1} d s \mu^{-1}(s)$. Then $m_{1}=\tilde{m}(1)=\left(d_{M} v\right) \bullet m_{0} \bullet\left(-\chi\left(\lambda_{1}\right)\right)$. In summary, if $\left(l_{0}, m_{0}\right)$ and $\left(m_{1}, l_{1}\right)$ are homotopy equivalent, then there exists

$$
\left(d v, \lambda_{1}\right) \in\left(d M^{-1} \otimes \mathfrak{m}_{A}\right) \times\left(L^{0} \otimes \mathfrak{m}_{A}\right)
$$

such that

$$
\left\{\begin{array}{l}
l_{1}=e^{\lambda_{1}} * l_{0}, \\
m_{1}=d \nu \bullet m_{0} \bullet\left(-\chi\left(\lambda_{1}\right)\right),
\end{array}\right.
$$

that is, $\left(l_{0}, m_{0}\right)$ and $\left(m_{1}, l_{1}\right)$ are gauge equivalent.

We now show that gauge implies homotopy. Assume $\left(l_{0}, m_{0}\right)$ and $\left(m_{1}, l_{1}\right)$ are gauge equivalent. Then there exists

$$
\left(d \nu, \lambda_{1}\right) \in\left(d M^{-1} \otimes \mathfrak{m}\right) \times\left(L^{0} \otimes \mathfrak{m}\right)
$$

such that

$$
\left\{\begin{array}{l}
l_{1}=e^{\lambda_{1}} * l_{0} \\
m_{1}=d \nu \bullet m_{0} \bullet\left(-\chi\left(\lambda_{1}\right)\right) .
\end{array}\right.
$$

Set $\tilde{l}(s, d s)=e^{s \lambda_{1}} * l_{0}$. By Corollary 7.3, $\tilde{l}$ satisfies the equation $d \tilde{l}+\frac{1}{2}[\tilde{l}, \tilde{l}]=0$. Set $\tilde{m}=(d(s v)) \bullet m_{0} \bullet\left(-\chi\left(s \lambda_{1}\right)\right)$. Reasoning as above, we find

$$
e^{\tilde{m}} * \chi(\tilde{l})=e^{d(s v)} * 0=0 .
$$


Therefore, $(\tilde{l}, \tilde{m})$ is a solution of the Maurer-Cartan equation in $C_{\chi}[s, d s]$. Moreover $\tilde{l}(0)=l_{0}, \tilde{l}(1)=l_{1}, \tilde{m}(0)=m_{0}$ and $\tilde{m}(1)=d \nu \bullet m_{0} \bullet\left(-\chi\left(\lambda_{1}\right)\right)=m_{1}$, that is, $\left(l_{0}, m_{0}\right)$ and $\left(m_{1}, l_{1}\right)$ are homotopy equivalent.

\section{Examples and applications}

Let $\chi: L \rightarrow M$ be a morphism of differential graded Lie algebras over a field $\mathbb{K}$ of characteristic 0. In the paper [Manetti 2005] one of the authors has introduced, having in mind the example of embedded deformations, the notion of MaurerCartan equation and gauge action for the triple $(L, M, \chi)$; these notions reduce to the standard Maurer-Cartan equation and gauge action of $L$ when $M=0$. More precisely, there are two functors of Artin rings $\mathrm{MC}_{\chi}, \operatorname{Def}_{\chi}:$ Art $\rightarrow$ Set, defined by

$M C_{\chi}(A)=$

$\left\{\left(x, e^{a}\right) \in\left(L^{1} \otimes \mathfrak{m}_{A}\right) \times \exp \left(M^{0} \otimes \mathfrak{m}_{A}\right): d x+\frac{1}{2}[x, x]=0, e^{a} * \chi(x)=0\right\}$,

$\operatorname{Def}_{\chi}(A)=\frac{\operatorname{MC}_{\chi}(A)}{\text { gauge equivalence }}$

where two solutions of the Maurer-Cartan equation are gauge equivalent if they belong to the same orbit of the gauge action

$$
\left(\exp \left(L^{0} \otimes \mathfrak{m}_{A}\right) \times \exp \left(d M^{-1} \otimes \mathfrak{m}_{A}\right)\right) \times \mathrm{MC}_{\chi}(A) \stackrel{*}{\longrightarrow} \mathrm{MC}_{\chi}(A)
$$

given by the formula

$$
\left(e^{l}, e^{d m}\right) *\left(x, e^{a}\right)=\left(e^{l} * x, e^{d m} e^{a} e^{-\chi(l)}\right)=\left(e^{l} * x, e^{d m \bullet a \bullet(-\chi(l))}\right) .
$$

The computations of Sections 6 and 7 show that $\mathrm{MC}_{\chi}$ and $\mathrm{Def}_{\chi}$ are canonically isomorphic to the functors $\mathrm{MC}_{\widetilde{C}(\chi)}$ and $\operatorname{Def}_{\widetilde{C}(\chi)}$ associated with the $L_{\infty}$ structure on $C_{\chi}$.

Example 8.1. Let $X$ be a compact complex manifold and let $Z \subset X$ be a smooth subvariety. Denote by $\Theta_{X}$ the holomorphic tangent sheaf of $X$ and by $N_{Z \mid X}$ the normal sheaf of $Z$ in $X$.

Consider the short exact sequence of complexes

$$
0 \rightarrow \operatorname{ker} \pi \stackrel{\chi}{\longrightarrow} A_{X}^{0, *}\left(\Theta_{X}\right) \stackrel{\pi}{\longrightarrow} A_{Z}^{0, *}\left(N_{Z \mid X}\right) \rightarrow 0 .
$$

It is proved in [Manetti 2005] that there exists a natural isomorphism between $\operatorname{Def}_{\chi}$ and the functor of embedded deformations of $Z$ in $X$. Therefore, the $L_{\infty}$ algebra $\widetilde{C}(\chi)$ governs the embedded deformations in this case. 
The DGLA $A_{Z}^{0, *}\left(\Theta_{Z}\right)$ governs the deformations of $Z$; the natural transformation

$$
\operatorname{Def}_{\widetilde{C}(\chi)}=\operatorname{Def}_{\chi} \rightarrow \operatorname{Def}_{A_{Z}^{0, *}\left(\Theta_{Z}\right)},
$$

$\{$ Embedded deformations of $Z\} \rightarrow\{$ Deformations of $Z$ \},

is induced by the morphism in DGLA ${ }^{2}$ given by the diagram

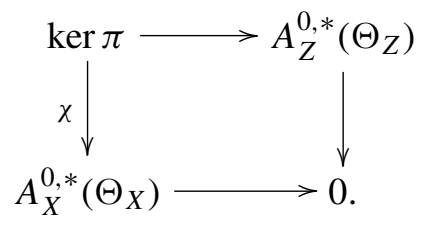

The next result was proved in [Manetti 2005] using the theory of extended deformation functors. Here we can prove it in a more standard way.

Theorem 8.2. Consider a commutative diagram

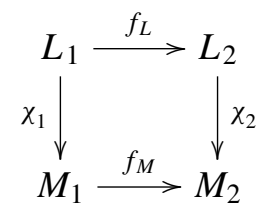

of morphisms of differential graded Lie algebras and assume that

$$
\left(f_{L}, f_{M}\right): C_{\chi_{1}} \rightarrow C_{\chi_{2}}
$$

is a quasiisomorphism of complexes (for example, if both $f_{L}$ and $f_{M}$ are quasiisomorphisms). Then the natural transformation $\operatorname{Def}_{\chi_{1}} \rightarrow \operatorname{Def}_{\chi_{2}}$ is an isomorphism. Proof. The map $\left(f_{L}, f_{M}\right): \widetilde{C}\left(\chi_{1}\right) \rightarrow \widetilde{C}\left(\chi_{2}\right)$ is a linear quasiisomorphism of $L_{\infty^{-}}$ algebras and then induces an isomorphism of the associated deformation functors [Kontsevich 2003].

Example 8.3. It is shown in [Fiorenza and Manetti 2006] how the $L_{\infty}$ structures $\widetilde{C}(\chi)$ are related to the period maps of a compact Kähler manifold $X$. Denote by $A_{X}=F^{0} \supseteq F^{1} \supseteq \cdots$, the Hodge filtration of differential forms on $X$, that is, for every $p \geq 0$,

$$
F^{p}=\bigoplus_{i \geq p} \bigoplus_{j} A_{X}^{i, j}
$$

For a fixed nonnegative integer $p$ one considers the inclusion of differential graded Lie algebras

$$
\left\{f \in \operatorname{Hom}^{*}\left(A_{X}, A_{X}\right): f\left(F^{p}\right) \subseteq F^{p}\right\} \stackrel{x}{\longrightarrow} \operatorname{Hom}^{*}\left(A_{X}, A_{X}\right) .
$$

The contraction of differential forms with vector fields

$$
i: A_{X}^{0, *}\left(\Theta_{X}\right) \rightarrow \operatorname{Hom}^{*}\left(A_{X}, A_{X}\right)[-1],
$$


and the holomorphic Lie derivative

$$
\boldsymbol{l}: A_{X}^{0, *}\left(\Theta_{X}\right) \rightarrow\left\{f \in \operatorname{Hom}^{*}\left(A_{X}, A_{X}\right): f\left(F^{p}\right) \subseteq F^{p}\right\}
$$

define a linear map $\mathfrak{p}^{p}=(\boldsymbol{l}, \boldsymbol{i}): A_{X}^{0, *}\left(\Theta_{X}\right) \rightarrow C_{\chi}$, which is actually a linear $L_{\infty^{-}}$ morphism

$$
\mathfrak{p}^{p}: A_{X}^{0, *}\left(\Theta_{X}\right) \rightarrow \widetilde{C}(\chi) .
$$

The induced morphism of deformation functors

$$
\mathscr{P}^{p}: \operatorname{Def}_{X} \rightarrow \operatorname{Def}_{\chi} \simeq \operatorname{Grass}_{H^{*}\left(F^{p}\right), H^{*}\left(A_{X}\right)}
$$

is the infinitesimal $p$-th period map of the Kähler manifold $X$. As immediate corollaries of this $L_{\infty}$-algebra interpretation of period maps, one recovers Griffiths' description of the differential of the period map, namely

$$
d \mathscr{P}^{p}=\boldsymbol{i}: H^{1}\left(X, T_{X}\right) \rightarrow \bigoplus_{i} \operatorname{Hom}\left(F^{p} H^{i}(X, \mathbb{C}), \frac{H^{i}(X, \mathbb{C})}{F^{p} H^{i}(X, \mathbb{C})}\right),
$$

and a proof of the so-called Kodaira's Principle [Clemens 2005; Manetti 2004a; Ran 1999] that obstructions to deformations of $X$ are contained in the kernel of

$$
i: H^{2}\left(X, T_{X}\right) \rightarrow \bigoplus_{i} \operatorname{Hom}\left(F^{p} H^{i}(X, \mathbb{C}), \frac{H^{i+1}(X, \mathbb{C})}{F^{p} H^{i+1}(X, \mathbb{C})}\right),
$$

for every $p \geq 0$.

Example 8.4. Let $\pi: A \rightarrow B$ be a surjective morphism of associative $\mathbb{K}$-algebras and denote by $I$ its kernel. The algebra $B$ is an $A$-module via $\pi$; this makes $B$ a trivial $I$-module. Let $K$ be the suspended Hochschild complex

$$
K=\operatorname{Hoch}^{\bullet}(I, B)[-1] .
$$

The differential $d$ of $K$ is identically zero if and only if $I \cdot I=0$.

The natural map

$$
\alpha: \operatorname{Hoch}^{\bullet}(A, A) \rightarrow K[1]=\operatorname{Hoch}^{\bullet}(I, B)
$$

is a surjective morphism of complexes, and its kernel

$$
\operatorname{ker} \alpha=\left\{f: f\left(I^{\otimes}\right) \subseteq I\right\}
$$

is a Lie subalgebra of $\operatorname{Hoch}^{\bullet}(A, A)$ endowed with the Hochschild bracket. Denote by $\chi: \operatorname{ker} \alpha \hookrightarrow \operatorname{Hoch}^{\bullet}(A, A)$ the inclusion. Since $\chi$ is injective, the projection on the second factor induces a quasiisomorphism of differential complexes

$$
\operatorname{pr}_{2}: C_{\chi} \rightarrow \operatorname{Coker}(\chi)[-1] \simeq K
$$


where the isomorphism on the right is induced by the map $\alpha$. Therefore we have a canonical $L_{\infty}$ structure (defined up to homotopy) on $K$. This gives a Lie structure on the cohomology of $K$, which is not trivial in general. Consider for instance the exact sequence

$$
0 \rightarrow \mathbb{K} \varepsilon \rightarrow \mathbb{K}[\varepsilon] /\left(\varepsilon^{2}\right) \stackrel{\pi}{\longrightarrow} \mathbb{K} \rightarrow 0
$$

and take $f \in K^{1}=H^{1}(K)$ with $f(\varepsilon)=1$. Choose as a lifting the linear map $g: \mathbb{K}[\varepsilon] /\left(\varepsilon^{2}\right) \rightarrow \mathbb{K}[\varepsilon] /\left(\varepsilon^{2}\right)$ such that $g(1)=0$ and $g(\varepsilon)=1$. Then

$$
d g(\varepsilon \otimes \varepsilon)=2 \varepsilon
$$

and so $d g \in \operatorname{ker} \alpha$. Therefore, $(d g, g)$ is a closed element of $C_{\chi}^{1}$ representing the cohomology class $f \in H^{1}(K)$ and so

$$
[f, f]=\alpha\left(\operatorname{pr}_{2}\left([(d g, g),(d g, g)]_{2}\right)\right)=\alpha([g, d g]) .
$$

One computes

$$
\begin{aligned}
{[f, f](\varepsilon \otimes \varepsilon) } & =\pi([g, d g](\varepsilon \otimes \varepsilon)) \\
& =\pi(g(d g(\varepsilon \otimes \varepsilon))-d g(g(\varepsilon) \otimes \varepsilon)+d g(\varepsilon \otimes g(\varepsilon))) \\
& =\pi(g(2 \varepsilon)-d g(1 \otimes \varepsilon)+d g(\varepsilon \otimes 1))=2 .
\end{aligned}
$$

Hence $[f, f] \neq 0$.

On the other hand, if $A=B \bigoplus I$ as an associative $\mathbb{K}$-algebra, then the $L_{\infty}$ structure on $K$ is trivial. Indeed, as $K[1]$ is considered to be a DGLA with trivial bracket, the obvious map

$$
K[1]=\operatorname{Hoch}^{\bullet}(I, B) \rightarrow \operatorname{Hoch}^{\bullet}(A, A)
$$

gives a commutative diagram of morphisms of DGLAs

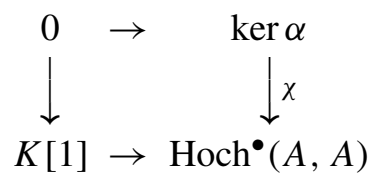

such that the composition $K \rightarrow C_{\chi} \rightarrow K$ is the identity. Therefore the $L_{\infty}$-algebra structure induced on $K$ is isomorphic to $\widetilde{C}(0 \hookrightarrow K[1])$, which is a trivial $L_{\infty^{-}}$ algebra.

\section{Acknowledgment}

We give our thanks to Jim Stasheff for precious comments on the first version of this paper and to the referee for very useful comments and suggestions, which greatly helped us in improving the exposition. 
The revised version of this paper was written while the second author was at Mittag-Leffler Institute in Stockholm, during a special year on Moduli Spaces; the author is grateful for the support received and for the warm hospitality.

\section{References}

[Bering 2006] K. Bering, "On non-commutative Batalin-Vilkovisky algebras, homotopy Lie algebras and the Courant bracket", preprint, 2006. hep-th/0603116

[Chen 1957] K.-T. Chen, "Integration of paths, geometric invariants and a generalized Baker-Hausdorff formula", Ann. of Math. (2) 65 (1957), 163-178. MR 19,12a Zbl 0077.25301

[Cheng and Getzler 2006] X. Z. Cheng and E. Getzler, "Homotopy commutative algebraic structures", preprint, 2006. math.AT/0610912

[Clemens 2005] H. Clemens, "Geometry of formal Kuranishi theory", Adv. Math. 198:1 (2005), 311-365. MR 2006h:32013 Zbl 1092.14004

[Dupont 1976] J. L. Dupont, "Simplicial de Rham cohomology and characteristic classes of flat bundles", Topology 15:3 (1976), 233-245. MR 54 \#1243 Zbl 0331.55012

[Dupont 1978] J. L. Dupont, Curvature and characteristic classes, Lecture Notes in Mathematics 640, Springer, Berlin, 1978. MR 58 \#18477 Zbl 0373.57009

[Fantechi and Manetti 1998] B. Fantechi and M. Manetti, "Obstruction calculus for functors of Artin rings. I”, J. Algebra 202:2 (1998), 541-576. MR 99f:14004 Zbl 0981.13009

[Fiorenza and Manetti 2006] D. Fiorenza and M. Manetti, " $L_{\infty}$ algebras, Cartan homotopies and period maps", preprint, 2006. math/0605297

[Fukaya 2003] K. Fukaya, "Deformation theory, homological algebra and mirror symmetry", pp. 121-209 in Geometry and physics of branes (Como, Italy, 2001), edited by U. Bruzzo et al., Ser. High Energy Phys. Cosmol. Gravit., IOP, Bristol, 2003. MR 2004c:14015

[Getzler 2004] E. Getzler, "Lie theory for nilpotent $L_{\infty}$-algebras", Ann. of Math. (2004). To appear. math/0404003v4

[Goldman and Millson 1990] W. M. Goldman and J. J. Millson, "The homotopy invariance of the Kuranishi space”, Illinois J. Math. 34:2 (1990), 337-367. MR 91d:32025 Zbl 0707.32004

[Grassi 1999] M. Grassi, "DG (co)algebras, DG Lie algebras and $L_{\infty}$ algebras", pp. 49-66 in Seminari di Geometria Algebrica (Pisa, 1998-99), Scuola Normale Superiore, Pisa, 1999. MR 1754794 [Huebschmann and Kadeishvili 1991] J. Huebschmann and T. Kadeishvili, "Small models for chain algebras", Math. Z. 207:2 (1991), 245-280. MR 92f:55029 Zbl 0723.57030

[Kadeishvili 1982] T. V. Kadeishvili, "The algebraic structure in the homology of an $A(\infty)$-algebra", Soobshch. Akad. Nauk Gruzin. SSR 108:2 (1982), 249-252 (1983). MR 84k:55009 Zbl 0535.55005 [Kontsevich 2003] M. Kontsevich, "Deformation quantization of Poisson manifolds", Lett. Math. Phys. 66:3 (2003), 157-216. MR 2005i:53122 Zbl 1058.53065 q-alg/9709040

[Kontsevich and Soibelman 2000] M. Kontsevich and Y. Soibelman, "Deformations of algebras over operads and the Deligne conjecture", pp. 255-307 in Conférence Moshé Flato (Dijon, 1999), vol. I, edited by G. Dito and D. Sternheimer, Math. Phys. Stud. 21, Kluwer, Dordrecht, 2000. MR 2002e:18012 Zbl 0972.18005 math.QA/0001151

[Kontsevich and Soibelman 2001] M. Kontsevich and Y. Soibelman, "Homological mirror symmetry and torus fibrations", pp. 203-263 in Symplectic geometry and mirror symmetry (Seoul, Korea, 2000), edited by K. Fukaya et al., World Sci., River Edge, NJ, 2001. MR 2003c:32025 Zbl 1072.14046 math.SG/0011041

[Lada and Markl 1995] T. Lada and M. Markl, "Strongly homotopy Lie algebras", Comm. Algebra 23:6 (1995), 2147-2161. MR 96d:16039 Zbl 0999.17019 hep-th/9406095

[Lada and Stasheff 1993] T. Lada and J. Stasheff, "Introduction to SH Lie algebras for physicists", Internat. J. Theoret. Phys. 32:7 (1993), 1087-1103. MR 94g:17059 Zbl 0824.17024 
[Manetti 1999] M. Manetti, "Deformation theory via differential graded Lie algebras", pp. 2148 in Seminari di Geometria Algebrica (Pisa, 1998-99), Scuola Normale Superiore, Pisa, 1999. MR 1754793

[Manetti 2004a] M. Manetti, "Cohomological constraint on deformations of compact Kähler manifolds”, Adv. Math. 186:1 (2004), 125-142. MR 2005e:32023 Zbl 1075.53074 math.AG/0105175

[Manetti 2004b] M. Manetti, "Lectures on deformations of complex manifolds (deformations from differential graded viewpoint)", Rend. Mat. Appl. (7) 24:1 (2004), 1-183. MR 2006a:32013 Zbl 1066.58010 math.AG/0507286

[Manetti 2005] M. Manetti, "Lie description of higher obstructions to deforming submanifolds", preprint, 2005. math.AG/0507287

[Merkulov 1999] S. A. Merkulov, "Strong homotopy algebras of a Kähler manifold", Internat. Math. Res. Notices 3 (1999), 153-164. MR 2000h:32026 Zbl 0995.32013 math.AG/9809172

[Merkulov 2005] S. A. Merkulov, "Operad of formal homogeneous spaces and Bernoulli numbers", preprint, 2005. 0708.0891v1

[Navarro Aznar 1987] V. Navarro Aznar, "Sur la théorie de Hodge-Deligne", Invent. Math. 90:1 (1987), 11-76. MR 88j:32037 Zbl 0639.14002

[Quillen 1969] D. Quillen, "Rational homotopy theory", Ann. of Math. (2) 90 (1969), 205-295. MR 41 \#2678 Zbl 0191.53702

[Ran 1999] Z. Ran, "Universal variations of Hodge structure and Calabi-Yau-Schottky relations", Invent. Math. 138:2 (1999), 425-449. MR 2000j:14021 Zbl 0956.14006

[Ran 2004] Z. Ran, "Lie atoms and their deformations", preprint, 2004. math.AG/0412204

[Remmert 1991] R. Remmert, Theory of complex functions, Graduate Texts in Mathematics 122, Springer, New York, 1991. MR 91m:30001 Zbl 0780.30001

[Rim 1972] D. S. Rim, "Formal deformation theory", pp. 32-132 (exposé VI) in Groupes de monodromie en géométrie algébrique, I (SGA 7 I) (Bois-Marie, 1967-1969), edited by A. Grothendieck, Lecture Notes in Math. 288, Springer, Berlin, 1972.

[Schlessinger 1968] M. Schlessinger, "Functors of Artin rings", Trans. Amer. Math. Soc. 130 (1968), 208-222. MR 36 \#184 Zbl 0167.49503

[Schlessinger and Stasheff 1979] M. Schlessinger and J. Stasheff, "Deformation theory and rational homotopy type", preprint, 1979.

[Schuhmacher 2004] F. Schuhmacher, "Deformation of $L_{\infty}$-algebras", preprint, 2004. math.QA/ 0405485

[Sullivan 2007] D. Sullivan, appendix to [Tradler and Zeinalian 2007], 2007.

[Tradler and Zeinalian 2007] T. Tradler and M. Zeinalian, "Infinity structure of Poincaré duality spaces”, Algebr. Geom. Topol. 7 (2007), 233-260. MR 2308943 math.AT/0309455

Communicated by Yuri Manin

Received 2007-04-03 Revised 2007-08-07 Accepted 2007-09-05

fiorenza@mat.uniroma1.it Dipartimento di Matematica "Guido Castelnuovo", Università di Roma "La Sapienza", Piazzale Aldo Moro 5, I-00185 Roma, Italy www.mat.uniroma1.it/ fiorenza/

manetti@mat.uniroma1.it Dipartimento di Matematica "Guido Castelnuovo", Università di Roma "La Sapienza", Piazzale Aldo Moro 5, I-00185 Roma, Italy www.mat.uniroma1.it/people/manetti/ 


\title{
Sur la définissabilité existentielle de la non-nullité dans les anneaux
}

\author{
Laurent Moret-Bailly
}

\begin{abstract}
On étudie les anneaux (notamment noethériens) dans lesquels l'ensemble des éléments non nuls est existentiel positif (réunion finie de projections d'ensembles « algébriques »). Dans le cas noethérien intègre, on montre notamment que cette condition est vérifiée pour tout anneau qui n'est pas local hensélien, et qu'elle ne l'est pas pour un anneau local hensélien excellent qui n'est pas un corps.

Ces résultats apportent au passage une réponse à une question de Popescu sur l'approximation forte pour les couples henséliens.
\end{abstract}

We investigate rings in which the set of nonzero elements is positive-existential (i.e., a finite union of projections of "algebraic" sets). In the case of Noetherian domains, we prove in particular that this condition is satisfied whenever the ring in question is not local Henselian, while it is not satisfied for any excellent local Henselian domain which is not a field.

As a byproduct, we obtain an answer to a question of Popescu on strong approximation for Henselian pairs.

\section{Introduction}

1.1. Définissabilité existentielle. Si $A$ est un anneau (commutatif unitaire) et $r$ un entier naturel, un sous-ensemble $Z$ de $A^{r}$ est dit [A-]existentiel (respectivement [A-]existentiel positif $)$ s'il existe une formule $\phi\left(t_{1}, \ldots, t_{r}\right)$ du langage des anneaux avec symboles de constantes pour les éléments de $A$, à $r$ variables libres, sans quantificateur universel $(\forall)$ (resp. et sans négation) telle que pour tout $\underline{t} \in A^{r}, \phi(\underline{t})$ soit vraie si et seulement si $\underline{t} \in Z$.

MSC2000: primary 13E05; secondary 03C99, 11U09, 13B40, 13J15.

Mots-clefs: Noetherian rings, positive-existential definability, Henselian rings, Artin approximation.

L'auteur est membre du réseau européen "Arithmetic Algebraic Geometry" (contrat HPRN-CT-200000120). 
Plus explicitement, un ensemble existentiel (resp. existentiel positif) est réunion d'une famille finie (éventuellement vide, voir remarque 1.1.1 ci-dessous) d'ensembles de la forme

$\left\{\underline{t} \in A^{r} \mid \exists \underline{x} \in A^{n}, F_{1}(\underline{t}, \underline{x})=\cdots=F_{s}(\underline{t}, \underline{x})=0 \wedge G_{1}(\underline{t}, \underline{x}) \neq 0 \wedge \cdots \wedge G_{u}(\underline{t}, \underline{x}) \neq 0\right\}$, resp.

$$
\left\{\underline{t} \in A^{r} \mid \exists \underline{x} \in A^{n}, F_{1}(\underline{t}, \underline{x})=\cdots=F_{s}(\underline{t}, \underline{x})=0\right\},
$$

où les $F_{j}$ et les $G_{j}$ sont des polynômes en $r+n$ indéterminées à coefficients dans $A$.

Si $f: A^{r} \rightarrow A^{s}$ est une application polynomiale (par exemple $A$-linéaire), l'image (resp. l'image réciproque) par $f$ de tout sous-ensemble existentiel de $A^{r}$ (resp. de $A^{s}$ ) est existentielle, et de même pour les sous-ensembles existentiels positifs. On voit en particulier que la notion de sous-ensemble existentiel (resp. existentiel positif) a un sens dans tout $A$-module libre de rang fini, indépendamment du choix d'une base.

1.1.1. Remarque. Dans la définition d'un ensemble existentiel positif, la réunion de la famille vide d'ensembles (*) donne naissance au sous-ensemble vide de $A^{r}$, qui est donc existentiel positif. Au niveau des formules, cette opération correspond à la disjonction de la famille vide de formules, qui est la constante logique FAUX. Il faut donc adjoindre celle-ci au langage des anneaux usuel, ce qui ne semble pas être de pratique courante en théorie des modèles.

Lorsque $A$ n'est pas nul, la constante FAUX est équivalente à la formule $1=0$, de sorte que l'on peut s'en dispenser. En revanche, si $A$ est l'anneau nul, la partie vide de $A^{r}$ n'est pas réunion d'une famille non vide d'ensembles $(*)$, ceux-ci n'étant jamais vides.

Le lecteur pourra, s'il y tient, revenir à la définition traditionnelle $\{+, ., 0,1\} \mathrm{du}$ langage des anneaux, au prix de modifications mineures (dans certains énoncés il faut se limiter aux anneaux non nuls).

1.2. La condition $(\boldsymbol{C})$. Pour $A$ donné, on peut se demander si tout ensemble existentiel dans $A^{r}$ est existentiel positif. Cette propriété équivaut manifestement à la condition suivante :

« l'ensemble $A \backslash\{0\}$ est existentiel positif »

1.2.1. Exemples. Il est clair que tout anneau fini vérifie (C) (y compris l'anneau nul, vu nos conventions).

Tout corps $K$ vérifie (C) $(t \in K$ est non nul si et seulement si il existe $x \in K$ tel que $t x=1$ ).

Il est aussi bien connu que $\mathbb{Z}$ vérifie (C) : par exemple, si $t \in \mathbb{Z}$, alors $t \neq 0$ si et seulement si il existe $x, y, w$ dans $\mathbb{Z}$ tels que $t w=(1+2 x)(1+3 y)$. La même astuce montre d'ailleurs que tout anneau d'entiers algébriques vérifie (C). 
L'anneau $A$ des entiers d'un corps local ne vérifie pas $(\mathrm{C})$ : en effet, tout sousensemble existentiel positif de $A$ est compact, alors que $A \backslash\{0\}$ ne l'est pas. Plus généralement, un anneau topologique compact infini ne vérifie pas (C).

Il est facile de voir qu'un produit $A_{1} \times A_{2}$ vérifie (C) si et seulement si $A_{1}$ et $A_{2}$ vérifient $(\mathrm{C})$. En revanche, un produit infini d'anneaux non nuls ne vérifie jamais (C) (tout ensemble existentiel positif est fermé pour le produit des topologies discrètes).

1.3. Résultats. Les principaux résultats de ce travail sont les suivants.

1.3.1. Anneaux noethériens. Soit $A$ un anneau noethérien. Alors :

- si $A$ est intègre et n'est pas local hensélien, il vérifie (C) (3.1);

- si $A$ est un localisé d'un anneau de Jacobson noethérien, il vérifie (C) (5.4);

- si $A$ est local hensélien excellent de dimension > 0, il ne vérifie pas (C) (4.1).

1.3.2. Propriétés d'approximation. Ces propriétés — et notamment les résultats de [Pfister et Popescu 1975; Denef et Lipshitz 1980; Popescu 1986; Swan 1998; Spivakovsky 1999] - jouent un rôle essentiel dans la preuve de 4.1 ; a contrario, on déduit de 3.1 que si un couple hensélien $(A, \mathfrak{r})$ vérifie la «propriété d'approximation forte », alors $A$ est nécessairement semi-local hensélien (sauf cas triviaux, comme $\mathfrak{r}=0$ ). Ce résultat (corollaire 4.2.1) précise la réponse négative donnée par Spivakovsky [1994] à une question de D. Popescu; voir la remarque 4.2.2.

1.3.3. Anneaux de fonctions holomorphes. Soit $X$ un espace analytique de Stein (par exemple un fermé analytique de $\mathbb{C}^{n}$ ou d'un polydisque ouvert), irréductible et réduit. Alors l'anneau des fonctions holomorphes sur $X$ vérifie $(\mathrm{C})$ (théorème $6.2)$.

1.4. Remarques sur les méthodes. Les deux éléments essentiels dans la démonstration du théorème 3.1 sont le lemme 3.2, qui suppose l'existence de deux idéaux premiers ayant certaines propriétés, et le lemme 3.3 qui permet de remplacer l'anneau étudié (muni d'un idéal premier p) par un autre ayant deux idéaux premiers au-dessus de $\mathfrak{p}$.

De ces deux lemmes, le premier généralise à peu de chose près l'argument utilisé plus haut pour $\mathbb{Z}(1.2 .1$, où les idéaux en question sont $2 \mathbb{Z}$ et $3 \mathbb{Z})$, dont des variantes ont été maintes fois utilisées dans la littérature (voir par exemple [Davis et al. 1976] ou, pour une généralisation récente, [Demeyer 2007], Proposition 2.3); l'idée du second remonte au moins à [Shlapentokh 1994], Theorem 4.2.

Quant au théorème 4.1, il repose comme on l'a dit sur les propriétés d'approximation, le lien étant la proposition 4.1.1. Le lien entre la condition (C) et l'approximation était déjà connu de T. Pheidas.

On voit donc que ce travail ne contient pas d'idée essentiellement nouvelle, l'auteur s'étant seulement efforcé de systématiser les méthodes existantes. 


\section{Généralités et rappels.}

2.1. Définissabilité. On laisse au lecteur la démonstration du lemme suivant :

2.1.1. Lemme. Soient $A$ un anneau, I un idéal de $A, \pi: A \rightarrow A / I$ l'homomorphisme canonique.

(i) Si I est de type fini, c'est un sous-ensemble existentiel positif de A.

(ii) On suppose que I est existentiel positif dans A. Si D est un sous-ensemble existentiel positif de $A / I$, alors $\pi^{-1}(D)$ est existentiel positif dans $A$.

En particulier, si A/I vérifie (C), alors $A \backslash I$ est existentiel positif dans $A$.

2.1.2. Lemme. Soient A un anneau, et B une A-algèbre qui est un A-module libre de rang fini $d$.

(i) Soit Z un sous-ensemble B-existentiel positif de B. Alors Z est A-existentiel positif (dans B vu comme A-module).

(ii) Si $d>0$ et si $B$ vérifie (C), alors $A$ vérifie $(\mathrm{C})$.

Démonstration. On déduit (i) de la remarque suivante : si $F: B^{r} \rightarrow B$ est définie par un polynôme à coefficients dans $B$, alors $F$ est définie par $d$ polynômes (en $r d$ indéterminées) à coefficients dans $A$, une fois $B$ identifié à $A^{d}$ par le choix d'une base. On en déduit (ii) en prenant $Z=B \backslash\{0\}$ et en remarquant que $A \backslash\{0\}$ est image réciproque de $Z$ par l'application $A$-linéaire canonique de $A$ dans $B$, qui est injective si $d>0$.

2.2. Anneaux locaux henséliens. On rappelle [Grothendieck 1967; Raynaud 1970] qu'un anneau local $A$, de corps résiduel $k$, est dit hensélien si toute $A$-algèbre finie (i.e. de type fini comme $A$-module) est un produit d'anneaux locaux. Une définition équivalente est la suivante : pour tout polynôme unitaire $P \in A[X]$ et tout élément $\bar{x}$ de $k$ qui est racine simple de (l'image de) $P$, il existe un unique $x \in A$ relevant $\bar{x}$ et annulant $P$.

Pour utiliser efficacement la première définition, nous aurons besoin du lemme suivant :

2.2.1. Lemme. Soit A un anneau semi-local noethérien. Les conditions suivantes sont équivalentes :

(i) A est isomorphe à un produit d'anneaux;

(ii) tout idéal premier de A est contenu dans un unique idéal maximal (autrement dit, tout quotient intègre de A est local);

(iii) tout idéal premier minimal de A est contenu dans un unique idéal maximal.

Démonstration. Les implications (i) $\Rightarrow$ (ii) $\Leftrightarrow$ (iii) sont faciles et laissées au lecteur. Supposons (iii), et montrons (i). Posons $X=\operatorname{Spec} A$, et soient $x_{1}, \ldots, x_{r}$ les points 
fermés de $X$ (correspondant aux idéaux maximaux $\mathfrak{m}_{1}, \ldots, \mathfrak{m}_{r}$ de $A$ ). Pour chaque $i \in\{1, \ldots, r\}$, notons $X_{i}$ le fermé de $X$ réunion des composantes irréductibles contenant $x_{i}$. L'hypothèse (iii) signifie que $X$ est réunion disjointe des $X_{i}$, qui sont donc ouverts dans $X$. Si l'on munit chaque $X_{i}$ de sa structure de sous-schéma ouvert de $X$, alors $X_{i}$ est local de point fermé $x_{i}$ et s'identifie donc à Spec $A_{\mathfrak{m}_{i}}$. Le fait que $X$ soit réunion disjointe des $X_{i}$ entraîne donc que $A \cong \prod_{i=1}^{r} A_{\mathfrak{m}_{i}}$, d'où la conclusion.

2.2.2. Corollaire. Soit A un anneau local noethérien. Les conditions suivantes sont équivalentes:

(i) A est hensélien;

(ii) toute A-algèbre finie intègre est un anneau local.

2.3. Anneaux excellents. On renvoie à [Grothendieck 1965] ou [Matsumura 1989] pour la définition des anneaux excellents. On en rappelle seulement quelques propriétés :

(i) tout anneau excellent est noethérien;

(ii) tout anneau local noethérien complet est excellent;

(iii) si $A$ est excellent, il en est de même de toute $A$-algèbre de type fini et de tout anneau de fractions de $A$;

(iv) si $A$ est local excellent, il en est de même de son hensélisé ;

(v) un anneau de valuation discrète $A$ est excellent si et seulement si le corps des fractions de son complété est une extension séparable du corps des fractions de $A$.

2.4. Propriétés d'approximation. Soient $A$ un anneau noethérien et $\mathfrak{r}$ un idéal de $A$. Pour tout $q \in \mathbb{N}$, on pose $A(q):=A / \mathfrak{r}^{q}$, et l'on note $\hat{A}=\lim _{q \in \mathbb{N}} A(q)$ le séparé complété r-adique de $A$.

Pour toute famille finie $S=\left(F_{j}\right)_{j=1, \ldots, s}$ de polynômes en $r$ indéterminées $X_{1}$, $\ldots, X_{r}$ à coefficients dans $A$, et pour toute $A$-algèbre $B$, on posera

$$
\operatorname{sol}(S, B):=\left\{\underline{x} \in B^{r} \mid F_{j}(\underline{x})=0 \text { pour tout } j\right\} .
$$

Il est clair que $\operatorname{sol}(S, B)$ est fonctoriel en $B$; il s'identifie à l'ensemble des morphismes de $A$-algèbres de $A[\underline{X}] /\left(F_{1}, \ldots, F_{S}\right)$ dans $B$.

On considérera la condition suivante («principe de Hasse infinitésimal ») :

$\operatorname{PHI}(A, \mathfrak{r}):$ " pour toute famille $S$ comme ci-dessus, $\operatorname{si} \operatorname{sol}(S, A(q)) \neq \varnothing$ pour tout $q \in \mathbb{N}$ alors $\operatorname{sol}(S, A) \neq \varnothing »$.

2.4.1. Remarques. (1) Il est facile de voir que $\operatorname{PHI}(A, \mathfrak{r})$ implique les propriétés suivantes : 
(i) $\mathfrak{r}$ est contenu dans le radical de $A$;

(ii) (conséquence de (i)) $A$ est r -adiquement séparé, i.e. $\bigcap_{q \in \mathbb{N}} \mathfrak{r}^{q}=\{0\}$;

(iii) pour tout idéal $J$ de $A, \operatorname{PHI}(A / J,(\mathfrak{r}+J) / J)$ est vérifiée ;

(iv) $(A, \mathfrak{r})$ vérifie la «propriété d'approximation »: pour $S$ comme ci-dessus, $\operatorname{sol}(S, A)$ est dense dans $\operatorname{sol}(S, \hat{A})$ pour la topologie $\mathfrak{r}$-adique;

(v) (conséquence de (iv)) ( $A, \mathfrak{r}$ ) est un couple hensélien : si $B$ est une $A$-algèbre étale telle que l'application $A / \mathfrak{r} \rightarrow B / \mathfrak{r} B$ soit un isomorphisme, alors il existe un $A$-homomorphisme de $B$ dans $A$.

(2) D'autre part, $\operatorname{PHI}(A, \mathfrak{r})$ est vraie dès que $(A, \mathfrak{r})$ vérifie la « propriété d'approximation forte $»$, que l'on peut formuler ainsi : pour $S$ comme ci-dessus et tout $q \in \mathbb{N}$, il existe $q^{\prime} \geq q$ tel que $\operatorname{sol}(S, A)$ et $\operatorname{sol}\left(S, A\left(q^{\prime}\right)\right)$ aient même image dans $\operatorname{sol}(S, A(q))$.

(3) Lorsque $A$ est local d'idéal maximal $\mathfrak{m}$, la propriété d'approximation pour $(A, \mathfrak{m})$ est en fait équivalente à la propriété d'approximation forte (et donc aussi à $\operatorname{PHI}(A, \mathfrak{m}))$, d'après [Pfister et Popescu 1975] (redémontré dans [Denef et Lipshitz 1980] par des méthodes de théorie des modèles).

(4) Enfin, lorsque $A$ est local hensélien excellent d'idéal maximal m, la propriété d'approximation est vérifiée, d'après [Popescu 1986] (autres références : [Swan 1998; Spivakovsky 1999]). Il en est donc de même de $\operatorname{PHI}(A, \mathfrak{m})$, et a fortiori de $\operatorname{PHI}(A, \mathfrak{r})$ pour tout idéal strict $\mathfrak{r}$. Retenons donc pour la suite :

2.5. Théorème. Soient $A$ un anneau local hensélien excellent, et $\mathfrak{r}$ un idéal strict de A. Alors la condition $\operatorname{PHI}(A, \mathfrak{r})$ est vérifiée.

\section{Anneaux noethériens intègres}

Dans ce paragraphe, nous allons établir le théorème suivant :

3.1. Théorème. Soit A un anneau intègre noethérien. Si A n'est pas local hensélien, alors A vérifie (C).

3.2. Lemme. Soient A un anneau intègre, $\mathfrak{p}_{1}$ et $\mathfrak{p}_{2}$ deux idéaux premiers de $A$. On suppose que:

(i) $A_{\mathfrak{p}_{1}}$ est noethérien;

(ii) $\mathfrak{p}_{1} \cap \mathfrak{p}_{2}$ ne contient aucun idéal premier non nul de A.

Alors, pour tout $t \in A$, on a l'équivalence:

$$
t \neq 0 \Longleftrightarrow \exists\left(w, x_{1}, x_{2}\right) \in A^{3},\left(t w=x_{1} x_{2} \wedge x_{1} \notin \mathfrak{p}_{1} \wedge x_{2} \notin \mathfrak{p}_{2}\right) .
$$

En outre, si $\mathfrak{p}_{1}$ et $\mathfrak{p}_{2}$ sont de type fini et si $A / \mathfrak{p}_{1}$ et $A / \mathfrak{p}_{2}$ vérifient $(\mathrm{C})$, alors $A$ vérifie (C). 
Démonstration. La dernière assertion résulte de la première en vertu du lemme 2.1.1 appliqué aux idéaux $\mathfrak{p}_{1}$ et $\mathfrak{p}_{2}$. Montrons la première assertion. L'implication $\Leftarrow$ est triviale puisque $A$ est intègre. Réciproquement, soit $t \neq 0$ dans $A$. Comme $A_{\mathfrak{p}_{1}}$ est noethérien, les idéaux premiers minimaux de $A_{\mathfrak{p}_{1}} /(t)$ sont en nombre fini; ils correspondent à un nombre fini d'idéaux premiers de $A$ contenant $t$ et contenus dans $\mathfrak{p}_{1}$, que nous noterons $\mathfrak{q}_{1}, \ldots, \mathfrak{q}_{r}$. L'hypothèse (ii) implique qu'aucun des $\mathfrak{q}_{j}$ n'est contenu dans $\mathfrak{p}_{2}$, ce qui implique que $\bigcap_{j} \mathfrak{q}_{j} \not \subset \mathfrak{p}_{2}$. Soit donc $y \in\left(\bigcap_{j} \mathfrak{q}_{j}\right) \backslash \mathfrak{p}_{2}$. L'image de $y$ dans $A_{\mathfrak{p}_{1}} /(t)$ appartient à tous les idéaux premiers minimaux donc est nilpotente : il existe $n \in \mathbb{N}$ et $v \in A_{\mathfrak{p}_{1}}$ tels que $y^{n}=t v$. Vu la définition du localisé $A_{\mathfrak{p}_{1}}$, il existe donc $s \in A \backslash \mathfrak{p}_{1}$ et $w \in A$ tels que $s y^{n}=t w$, de sorte que $t$ vérifie la propriété voulue (avec $x_{1}=s$ et $x_{2}=y^{n}$ : noter que $y^{n} \notin \mathfrak{p}_{2}$ puisque $\left.y \notin \mathfrak{p}_{2}\right)$.

\subsubsection{Remarques.}

(1) La condition (i) de l'énoncé du lemme peut être affaiblie en « l'espace topologique $\operatorname{Spec}\left(A_{\mathfrak{p}_{1}}\right)$ est noethérien $»$; plus précisément on n'utilise que le fait que pour tout $t \in A_{\mathfrak{p}_{1}}$, l'anneau $A_{\mathfrak{p}_{1}} /(t)$ n'a qu'un nombre fini d'idéaux premiers minimaux.

(2) La condition (ii) est notamment vérifiée si l'on a $\operatorname{dim} A_{\mathfrak{p}_{1}}=1$ et $\mathfrak{p}_{1} \not \subset \mathfrak{p}_{2}$ (ou l'inverse).

Voici un corollaire immédiat de 3.2 :

3.2.2. Corollaire. Soit $R$ un anneau intègre vérifiant $(\mathrm{C})$. Alors $R[X]$ vérifie $(\mathrm{C})$.

Démonstration. Il suffit d'appliquer la dernière assertion de 3.2 avec $A=R[X]$, $\mathfrak{p}_{1}=(X)$ et $\mathfrak{p}_{2}=(X-1)$.

3.3. Lemme. Soit A un anneau intègre noethérien, de corps des fractions $K$. Soit $\mathfrak{p}$ un idéal premier non nul de A; on suppose que $\mathfrak{p} n$ 'est pas le plus grand idéal premier de A (autrement dit, A n'est pas un anneau local d'idéal maximal $\mathfrak{p}$ ).

Alors il existe un polynôme $F=X^{2}+a X+b \in A[X]$ ayant les propriétés suivantes:

(i) $a \notin \mathfrak{p}$;

(ii) $b \in \mathfrak{p}$;

(iii) $F$ est irréductible dans $K[X]$.

Démonstration. L'hypothèse sur $\mathfrak{p}$ entraîne qu'il existe un élément non inversible $\alpha$ de $A \backslash\{0\}$ qui n'appartient pas à $\mathfrak{p}$. Soit $\mathfrak{q}$ un idéal premier minimal parmi ceux contenant $\alpha$ : alors $\mathfrak{q} \not \subset \mathfrak{p}$ (puisque $\alpha \in \mathfrak{q}$ ), et l'anneau $A_{\mathfrak{q}}$ est local noethérien de dimension 1 (vu la minimalité de q). Comme $\mathfrak{p}$ n'est pas nul cela entraîne que $\mathfrak{p} \not \subset \mathfrak{q}$. Fixons $\beta \in \mathfrak{p} \backslash \mathfrak{q}$. Nous avons ainsi trouvé un idéal premier $\mathfrak{q}$ et deux éléments $\alpha$ et $\beta$ de $A$ vérifiant :

$$
\alpha \in \mathfrak{q} \backslash \mathfrak{p} ; \quad \beta \in \mathfrak{p} \backslash \mathfrak{q} ; \quad \operatorname{dim} A_{\mathfrak{q}}=1 .
$$


Il résulte du théorème de Krull-Akizuki [Bourbaki 1965, §2, nº 5, prop. 5 et cor. 2] que la clôture intégrale de $A_{\mathfrak{q}}$ est un anneau de Dedekind; si on le localise en l'un de ses idéaux maximaux, on obtient un anneau de valuation discrète de corps des fractions $K$ qui domine $A_{\mathfrak{q}}$. Notons $v: K \rightarrow \mathbb{Z} \cup\{+\infty\}$ la valuation normalisée correspondante. Comme le monoïde $v(A \backslash\{0\})$ engendre le groupe $\mathbb{Z}$, il existe $\gamma \in A$ tel que $v(\gamma)$ soit impair et positif.

Posons $b:=\beta \gamma$. Alors $b \in \mathfrak{p}$ et $v(b)$ est impair. Pour $n \in \mathbb{N}$ convenable, l'élément $a:=\alpha^{n}$ vérifie $v(a)>v(b) / 2$ (et évidemment $a \notin \mathfrak{p}$ ).

Il reste à montrer que $F=X^{2}+a X+b$ n'a pas de racine dans $K$. Si $z$ était une telle racine, on aurait $z(z+a)=-b$. Si $v(z)<v(a)$ ceci entraîne $v(z(z+a))=$ $2 v(z)=v(b)$, impossible puisque $v(b)$ est impair; sinon, on a $v(z) \geq v(a)$ donc $v(z(z+a)) \geq 2 v(a)>v(b)$ vu le choix de $a$ : nouvelle contradiction. (Les amateurs de polygones de Newton se contenteront de remarquer que celui de $F$ a pour seule pente $-v(b) / 2$, qui n'est pas un entier).

3.3.1. Remarque. On voit notamment que, sous les hypothèses de 3.3, l'anneau local $A_{\mathfrak{p}}$ n'est pas hensélien, puisque $F$ est irréductible mais a deux racines simples dans le corps résiduel de $A_{\mathfrak{p}}$.

3.4. Proposition. Soit A un anneau intègre noethérien. On suppose qu'il existe un idéal premier $\mathfrak{p}$ de A tel que:

(i) $\mathfrak{p}$ n'est pas le plus grand idéal premier de A;

(ii) $A / \mathfrak{p}$ vérifie (C).

Alors A vérifie (C).

Démonstration. On procède par récurrence sur $h:=\operatorname{dim} A_{\mathfrak{p}}$. Si $h=0$, alors $\mathfrak{p}$ est nul et l'assertion est triviale.

Supposons $h>0$. Il existe alors un polynôme $F=X^{2}+a X+b$ comme dans le lemme 3.3. Soit $B$ la $A$-algèbre $A[X] /(F)$. Alors $B$ est intègre, et est un $A$-module libre de rang 2 ; il suffit donc (lemme 2.1.2) de montrer que $B$ vérifie (C). L'anneau $B / \mathfrak{p} B$ est isomorphe à $(A / \mathfrak{p})[X] /(X(X+\bar{a}))$ où $\bar{a} \neq 0$ est la classe de $a$ modulo $\mathfrak{p}$. Donc $B$ a deux idéaux premiers distincts au-dessus de $\mathfrak{p}$, à savoir $\mathfrak{p}_{1}=\mathfrak{p} B+x B$ et $\mathfrak{p}_{2}=\mathfrak{p} B+(x+a) B$ où $x$ est la classe de $X$. Comme $B$ est fini libre sur $A$, on a $\operatorname{dim} B_{\mathfrak{p}_{1}}=\operatorname{dim} B_{\mathfrak{p}_{2}}=h$. De plus $B / \mathfrak{p}_{1} \cong A / \mathfrak{p}$ (l'isomorphisme envoie la classe de $x$ sur 0 ) et de même $B / \mathfrak{p}_{2} \cong A / \mathfrak{p}$ (l'isomorphisme envoie la classe de $x$ sur $-\bar{a}$ ). En particulier les anneaux $B / \mathfrak{p}_{1}$ et $B / \mathfrak{p}_{2}$ vérifient $(C)$. Distinguons deux cas :

(1) $\mathfrak{p}_{1} \cap \mathfrak{p}_{2}$ ne contient aucun idéal premier non nul de $B$ (condition vérifiée en particulier si $h=1)$ : alors on déduit du lemme 3.2 que $B$ vérifie $(C)$.

(2) il existe un idéal premier $\mathfrak{q} \subset \mathfrak{p}_{1} \cap \mathfrak{p}_{2}$ non nul : en choisissant $\mathfrak{q}$ minimal on peut supposer que $\operatorname{dim} B_{\mathfrak{q}}=1$. Pour $i \in\{1,2\}$ posons $\overline{\mathfrak{p}}_{i}:=\mathfrak{p}_{i} / \mathfrak{q} \subset \bar{B}:=B / \mathfrak{q}$. Alors les localisés $\bar{B}_{\overline{\mathfrak{p}}_{i}}$ sont de dimension $\leq h-1$; en outre $\overline{\mathfrak{p}}_{1} \not \subset \overline{\mathfrak{p}}_{2}$, donc $\overline{\mathfrak{p}}_{2}$ n'est pas le 
plus grand idéal premier de $\bar{B}$ et l'hypothèse de récurrence s'applique à $\bar{B}$. Ainsi $\bar{B}$ vérifie $(\mathrm{C})$, et donc $B$ aussi d'après le cas $h=1$ déjà établi.

3.5. Démonstration du théorème 3.1. Soit $A$ comme dans l'énoncé. Si $A$ n'est pas local, il suffit d'appliquer 3.4 en prenant pour $\mathfrak{p}$ n'importe quel idéal maximal de $A$ : il est clair que la condition (ii) de la proposition est vérifiée puisque $A / \mathfrak{p}$ est un corps.

Supposons $A$ local mais non hensélien. Il existe alors une $A$-algèbre finie intègre $B_{0}$ qui n'est pas locale, d'après 2.2.2. Soit $\left(\xi_{1}, \ldots, \xi_{s}\right)$ une famille génératrice finie du $A$-module $B_{0}$, et pour chaque $i \in\{1, \ldots, s\}$ soit $F_{i} \in A\left[X_{i}\right]$ un polynôme unitaire, en une indéterminée $X_{i}$, annulant $\xi_{i}$. Alors $B_{0}$ est quotient de $B:=A\left[X_{1}, \ldots, X_{s}\right] /\left(F_{1}, \ldots, F_{s}\right)$ qui est libre de rang fini comme $A$-module.

Nous avons donc trouvé une $A$-algèbre finie libre $B$ et un idéal premier $\mathfrak{p}$ de $B$ tel que $B_{0}=B / \mathfrak{p}$ ne soit pas local. Soit $\mathfrak{q}$ un idéal premier minimal de $B$ contenu dans $\mathfrak{p}$ : alors $B / \mathfrak{q}$ n'est pas local donc vérifie $(C)$. Par suite $B \backslash \mathfrak{q}$ est existentiel positif dans $B$ (2.1.1). Soit $j: A \rightarrow B$ le morphisme structural. Comme les éléments de $\mathfrak{q}$ sont diviseurs de zéro dans $B$, que $A$ est intègre et que $B$ est $A$-libre on a $j^{-1}(\mathfrak{q})=\{0\}$. Donc $A \backslash\{0\}=j^{-1}(B \backslash \mathfrak{q})$ est existentiel positif dans $A$, ce qui achève la démonstration.

3.6. Anneaux de fractions. Une conséquence du théorème 3.1 est que pour les anneaux noethériens intègres, la propriété $(\mathrm{C})$ est « stable par anneaux de fractions ». Plus précisément :

3.6.1. Corollaire. Soit A un anneau intègre noethérien, et soit $S$ une partie multiplicative de A. On suppose vérifiée l'une des conditions suivantes:

(i) $S \not \subset A^{\times}$(de sorte que $S^{-1} A \neq A$ );

(ii) A vérifie (C).

Alors $S^{-1} A$ vérifie (C).

Démonstration. Si $0 \in S$, alors $S^{-1} A$ est nul et tout est trivial. On suppose donc que $0 \notin S$, de sorte que $S^{-1} A$ est noethérien et intègre. S'il n'est pas local, on conclut par 3.1. Supposons-le local : il est donc de la forme $A_{\mathfrak{p}}$ où $\mathfrak{p}$ est un idéal premier de $A$. Si p est nul, alors $S^{-1} A$ est un corps ; si $A$ est local d'idéal maximal $\mathfrak{p}$, alors $S^{-1} A=A$, ce qui est exclu dans le cas (i) et implique le résultat dans le cas (ii). Sinon, nous sommes dans la situation du lemme 3.3, de sorte que $A_{\mathfrak{p}}$ n'est pas hensélien d'après 3.3.1, donc vérifie $(\mathrm{C})$.

\section{Le cas hensélien}

4.1. Théorème. Soient $A$ un anneau noethérien, $\mathfrak{r}$ un idéal non contenu dans le nilradical de A. Si la propriété $\operatorname{PHI}(A, \mathfrak{r})$ de 2.4 est vérifiée (et notamment si A est local hensélien excellent, d'après 2.5), alors A ne vérifie pas (C). 
Démonstration. L'hypothèse sur $\mathfrak{r}$ équivaut à dire que la topologie $\mathfrak{r}$-adique de $A$ n'est pas discrète. Donc $A \backslash\{0\}$ n'est pas fermé dans $A$ pour cette topologie, de sorte que le théorème résulte de la proposition 4.1.1 qui suit.

4.1.1. Proposition. Soit A un anneau noethérien, $\mathfrak{r}$ un idéal de A. Les conditions suivantes sont équivalentes:

(i) $\operatorname{PHI}(A, \mathfrak{r})$ est vérifiée;

(ii) pour tout $n \in \mathbb{N}$, toute partie existentielle positive de $A^{n}$ est fermée pour la topologie $\mathfrak{r}$-adique.

Démonstration. (i) $\Rightarrow$ (ii) : supposons $\operatorname{PHI}(A, \mathfrak{r})$ vérifiée, et soit $Z \subset A^{n}$ un sousensemble existentiel positif. Alors $Z$ est réunion finie d'ensembles de la forme

$$
\left\{\underline{t} \in A^{n} \mid(\exists \underline{x}) F_{1}(\underline{t}, \underline{x})=\ldots=F_{S}(\underline{t}, \underline{x})=0\right\}
$$

où chaque $F_{j}$ est un polynôme à coefficients dans $A$ en $n+r$ variables (où $\underline{x}=$ $\left.\left(x_{1}, \ldots, x_{r}\right)\right)$. Pour voir que $Z$ est fermé, on peut donc supposer qu'il est de la forme ci-dessus.

Soit $\underline{t} \in A^{n}$ adhérent à $Z$. Pour tout $q \in \mathbb{N}$, il existe donc $(\underline{t}(q), \underline{x}(q)) \in A^{n+r}$ tels que $\underline{t}-\underline{t}(q) \in \mathfrak{r}^{q} A^{n+r}$ et que $F_{j}(\underline{t}(q), \underline{x}(q))=0$ pour tout $j$. Comme les $F_{j}$ sont à coefficients dans $A$, on a donc $F_{j}(\underline{t}, \underline{x}(q)) \in \mathfrak{r}^{q}$. Autrement dit, le système d'équations

$$
F_{j}\left(\underline{t}, X_{1}, \ldots, X_{r}\right)=0 \quad(j=1, \ldots, s)
$$

en les inconnues $X_{i}$ admet une solution modulo $\mathfrak{r}^{q}$ pour tout $q$. L'hypothèse implique donc qu'il a une solution dans $A^{r}$, donc que $\underline{t} \in Z$, cqfd.

(ii) $\Rightarrow$ (i) : supposons (ii), et reprenons les notations de 2.4. On a donc une famille finie $S=\left(F_{j}\right)_{j=1, \ldots, s}$ de polynômes en $r$ indéterminées à coefficients dans $A$. Supposons que $\operatorname{sol}(S, A(q)) \neq \varnothing$ pour tout $q \in \mathbb{N}$, et considérons l'ensemble $Z \subset A^{s}$ image de l'application $\left(F_{1}, \ldots, F_{s}\right): A^{r} \rightarrow A^{s}$. Alors $Z$ est existentiel positif, et l'hypothèse sur $S$ signifie que $0 \in A^{s}$ est adhérent à $Z$. Comme $Z$ est fermé d'après (ii), on conclut que $0 \in Z$, donc que $\operatorname{sol}(S, A) \neq \varnothing$.

4.2. Application à une question de D. Popescu. En combinant 4.1 et 3.1 , on obtient :

4.2.1. Corollaire. Soient $A$ un anneau intègre noethérien, $\mathfrak{r}$ un idéal non nul de A. $\operatorname{Si} \operatorname{PHI}(A, \mathfrak{r})$ est vérifiée, alors A est local hensélien.

4.2.2. Remarque. En utilisant la remarque 2.4.1, on en déduit plus généralement que si $A$ est un anneau noethérien, $\mathfrak{r}$ un idéal de $A$ non contenu dans un idéal premier minimal, et si $\operatorname{PHI}(A, \mathfrak{r})$ est vérifiée, alors $A$ est semi-local hensélien (donc produit d'anneaux locaux henséliens). 
Popescu demandait dans [Popescu 1986] si tout couple hensélien $(A, \mathfrak{r})(c f$. 2.4.1(1)(v)) tel que l'homomorphisme de complétion $A \rightarrow \hat{A}$ soit régulier vérifie l'approximation forte, et Spivakovsky [1994] a montré par un exemple que la réponse est en général négative. Le résultat ci-dessus montre que la réponse est toujours négative, sauf dans le cas semi-local (où elle est affirmative, au moins lorsque $\mathfrak{r}$ est le radical ; j'ignore ce qui se passe par exemple si $A$ est local hensélien excellent et si $\mathfrak{r}$ est différent de l'idéal maximal).

On a une réciproque partielle à 4.1 :

4.3. Théorème. Soit A un anneau local noethérien hensélien, intègre et de dimension 1, d'idéal maximal $\mathfrak{m}$. Si $\mathrm{PHI}(A, \mathfrak{m})$ n'est pas vérifiée, alors A vérifie $(\mathrm{C})$.

Démonstration. Par hypothèse il existe un système $S=\left(F_{j}\right)_{j=1, \ldots, s}$ comme dans 2.4 qui a un zéro modulo $\mathfrak{m}^{q}$ pour tout $q \in \mathbb{N}$ mais n'a pas de zéro dans $A^{r}$.

Montrons qu'il existe un tel système réduit à un seul polynôme : en effet, comme $\operatorname{dim} A>0$, le corps des fractions $K$ de $A$ n'est pas algébriquement clos (il admet une valuation discrète non triviale, cf. la preuve de 3.3) et il existe donc un polynôme $P \in K\left[X_{1}, \ldots, X_{s}\right]$ ayant $(0, \ldots, 0)$ pour seul zéro dans $K^{s}$. On peut naturellement prendre $P$ dans $A[\underline{X}]$; le polynôme composé $F:=P\left(F_{1}, \ldots, F_{s}\right)$ a alors un zéro modulo $\mathfrak{m}^{q}$ pour tout $q \in \mathbb{N}$ mais n'a pas de zéro dans $A^{r}$.

Montrons alors que pour $x \in A$ quelconque on a l'équivalence :

$$
x \neq 0 \quad \Longleftrightarrow \quad(\exists t)(\exists w) x w=F(t) .
$$

L'implication $\Leftarrow$ résulte du fait que $F$ n'a pas de zéro dans $A^{r}$; réciproquement, si $x \neq 0$, l'anneau $A / x A$ est artinien donc il existe $q \in \mathbb{N}$ tel que $\mathfrak{m}^{q} \subset x A$; vu le choix de $F$, il existe $t \in A$ tel que $F(t) \in \mathfrak{m}^{q}$, donc $F(t) \in x A$, cqfd.

4.4. Un exemple. Nous donnons ici un exemple, tiré de [Bosch et al. 1990], d'anneau de valuation discrète hensélien qui ne vérifie pas (PHI). Soit $k$ un corps de caractéristique $p>0$. Choisissons un élément $\xi$ de $k \llbracket T]$ qui est transcendant sur $k(T)$; posons

$$
U=\xi^{p} \quad \text { et } \quad A_{0}:=k[[T]] \cap k(T, U) .
$$

Enfin soit $A$ le hensélisé de $A_{0}$. On vérifie facilement que $A_{0}$ et $A$ sont des anneaux de valuation discrète, de complété $k \llbracket[T]$. De plus, par construction, $\xi^{p} \in A_{0}$ mais $\xi \notin A_{0}$ et donc $\xi \notin A$ puisque le corps des fractions de $A$ est séparable sur celui de $A_{0}$. L'équation $X^{p}=U$ n'a donc pas de solution dans $A$, mais en a une dans $A /\left(T^{q}\right)$ pour tout $q>0$ (à savoir la classe d'un polynôme en $T$ congru à $\xi$ modulo $\left.T^{q}\right)$.

En particulier $A$ vérifie (C) : explicitement, pour tout $f \in A$, on a $f \neq 0$ si et seulement si il existe $g$ et $h$ dans $A$ tels que $f g=h^{p}-U$. 


\section{Anneaux noethériens non intègres}

5.1. Notations. La condition (C), à la connaissance de l'auteur, n'a été utilisée dans la littérature que dans le cas intègre; pour les anneaux plus généraux, nous nous contenterons donc de quelques remarques élémentaires. Notons d'abord que pour un anneau non intègre il est naturel de considérer, outre $A \backslash\{0\}$, l'ensemble $A^{\text {reg }}$ des éléments réguliers (c'est-à-dire « non diviseurs de zéro ») de $A$, qui est contenu dans $A \backslash\{0\}$ si $A$ n'est pas nul et lui est égal si $A$ est intègre. Ceci conduit à envisager, pour un anneau $A$ donné, la condition

$\left(\mathrm{C}^{\text {reg }}\right) \quad$ «l'ensemble $A^{\text {reg }}$ est existentiel positif dans $A$ »

qui est équivalente à $(C)$ si $A$ est intègre. Nous utiliserons en outre la condition « tout quotient intègre de $A$ vérifie $(\mathrm{C})$ ».

5.2. Proposition. Soit A un anneau noethérien. On fait l'une des hypothèses suivantes:

(i) A vérifie $(\mathrm{C})$;

(ii) pour tout idéal premier $\mathfrak{p}$ associé à $A$, le quotient $A / \mathfrak{p}$ vérifie $(\mathrm{C})$.

Alors A vérifie $\left(\mathrm{C}^{\mathrm{reg}}\right)$, et vérifie $(\mathrm{C})$ s'il est réduit.

Démonstration. Soient $\mathfrak{p}_{1}, \ldots, \mathfrak{p}_{n}$ les idéaux premiers associés à $A$. Par définition, chaque $\mathfrak{p}_{i}$ est l'annulateur d'un élément $\alpha_{i}$ de $A$; on sait en outre [Bourbaki 1961, chapitre IV, $\S 1, \mathrm{n}^{\circ} 1$, cor. 3 de la prop. 2] que $A \backslash A^{\text {reg }}$ est la réunion des $\mathfrak{p}_{i}$, de sorte que

$$
A^{\mathrm{reg}}=\bigcap_{i=1}^{n}\left(A \backslash \mathfrak{p}_{i}\right) .
$$

Dans le cas (ii), chaque $A \backslash \mathfrak{p}_{i}$ est existentiel positif par 2.1.1, et il en est de même dans le cas (i) puisque $A \backslash \mathfrak{p}_{i}=\left\{t \in A \mid t \alpha_{i} \neq 0\right\}$. Donc $A$ vérifie $\left(\mathrm{C}^{\text {reg }}\right)$. Si $A$ est réduit, il suffit de remarquer que les $\mathfrak{p}_{i}$ sont les idéaux premiers minimaux de $A$ et que $A \backslash\{0\}$ est la réunion des $A \backslash \mathfrak{p}_{i}$.

5.3. Théorème. Soit $A$ un anneau noethérien vérifiant $(\mathrm{Q})$, et soit $S$ une partie multiplicative de A. Alors:

(i) A vérifie (C);

(ii) $S^{-1} A$ vérifie (Q) (et donc (C), d'après (i)).

Démonstration. (i) d'après [Bourbaki 1961, chap. IV, §1, nº 4, théorème 1] il existe une suite d'idéaux

$$
A=I_{0} \supset I_{1} \supset \cdots \supset I_{n}=\{0\}
$$

et des idéaux premiers $\mathfrak{p}_{0}, \ldots, \mathfrak{p}_{n-1}$ tels que, pour tout $j$, le $A$-module $I_{j} / I_{j+1}$ soit isomorphe à $A / \mathfrak{p}_{j}$; fixons pour chaque $j$ un élément $\alpha_{j}$ de $I_{j}$ engendrant $I_{j} / I_{j+1}$. 
Pour tout $x \in A$, on a alors les équivalences

$$
\begin{aligned}
x \neq 0 & \Longleftrightarrow \bigvee_{j=0}^{n-1}\left(x \in I_{j} \backslash I_{j+1}\right) \\
& \Longleftrightarrow \bigvee_{j=0}^{n-1}(\exists t)(\exists u)\left(x=t \alpha_{j}+u \wedge t \in A \backslash \mathfrak{p}_{j} \wedge u \in I_{j+1}\right)
\end{aligned}
$$

d'où la conclusion puisque $A \backslash \mathfrak{p}_{j}$ et $I_{j+1}$ sont existentiels positifs, d'après l'hypothèse sur $A$ et le lemme 2.1.1.

(ii) Les quotients intègres de $S^{-1} A$ sont des anneaux de fractions de quotients intègres de $A$, donc le cas (ii) de 3.6.1 entraîne que $S^{-1} A$ vérifie (Q).

Rappelons qu'un anneau $A$ est un anneau de Jacobson si tout idéal premier de $A$ est intersection d'idéaux maximaux.

5.4. Corollaire. Tout anneau de Jacobson noethérien vérifie (Q).

Par suite, d'après 5.3, tout anneau de fractions d'un anneau de Jacobson noethérien vérifie $(\mathrm{C})$.

En particulier:

- tout anneau artinien vérifie $(\mathrm{C})$;

- soit $k$ un corps ou un anneau de Dedekind ayant une infinité d'idéaux maximaux; alors toute $k$-algèbre essentiellement de type fini vérifie (C).

Démonstration. Il est clair qu'un anneau de Jacobson intègre et local est un corps, de sorte que, d'après 3.1, tout anneau de Jacobson intègre et noethérien vérifie (C). Comme il est non moins clair qu'un quotient d'un anneau de Jacobson est de Jacobson, on en déduit la première assertion, qui entraîne les autres.

\section{Fonctions analytiques.}

Pour les notions de base sur les espaces analytiques et les espaces de Stein, le lecteur pourra consulter [Grauert et Remmert 1979]. Un espace analytique sera toujours supposé séparable (en particulier l'ensemble de ses composantes irréductibles est dénombrable) et de dimension finie.

Parmi les espaces de Stein on compte notamment les espaces $\mathbb{C}^{n}$, les polydisques, et les sous-espaces analytiques fermés de ceux-ci.

$\mathrm{Si}\left(X, \mathcal{O}_{X}\right)$ est un espace analytique, nous noterons $\mathscr{H}(X)$ l'anneau $H^{0}\left(X, \mathcal{O}_{X}\right)$ des fonctions holomorphes globales sur $X$.

6.1. Proposition. Soit $\left(X, \mathscr{O}_{X}\right)$ un espace analytique de Stein, irréductible et réduit, de dimension $>0$, et soit $P$ un point de $X$. Il existe un sous-espace fermé $Y$ de $X$ ayant les propriétés suivantes:

(i) $P \notin Y$, et $Y$ est disjoint du lieu singulier de $X$;

(ii) $Y$ est irréductible et réduit, et son idéal $\oiint_{Y} \subset \mathcal{O}_{X}$ est localement principal; 
(iii) l'idéal $\mathfrak{p}=H^{0}\left(X, \Phi_{Y}\right)$ de l'anneau $A:=\mathscr{H}(X)$ formé des fonctions holomorphes sur $X$ nulles sur $Y$ est de type fini;

(iv) avec les notations de (iii), l'anneau $A_{\mathfrak{p}}$ est un anneau de valuation discrète.

Démonstration. Soit $Z \subset X$ le sous-espace fermé réunion du lieu singulier de $X$ et du point $P$. Vu les hypothèses sur $X$ il existe un point $Q \in X \backslash Z$. Comme $X$ est de Stein il existe une fonction holomorphe $f$ sur $X$ qui vaut 1 sur $Z, 0$ en $Q$ et dont la différentielle en $Q$ n'est pas nulle. Le sous-espace fermé $Y^{\prime}$ de $X$ défini par $f=0$ est un diviseur contenu dans $X \backslash Z$, et lisse au voisinage de $Q$. Soit $Y$ l'unique composante irréductible de $Y^{\prime}$ contenant $Q$. Il est clair que $Y$ vérifie les conditions (i), (ii) et (iv), et la propriété (iii) résulte du lemme 6.1.2 ci-dessous (appliqué à $\left.\mathscr{F}=\Phi_{Y}\right)$.

6.1.1. Notations. Soit $\left(X, \mathrm{O}_{X}\right)$ un espace analytique, et soit $\mathscr{F}$ un $\mathrm{O}_{X}$-module cohérent. Pour tout $x \in X$ notons $\mathscr{F}_{x}$ le $\mathcal{O}_{X, x}$-module de type fini fibre de $\mathscr{F}_{\mathrm{F}}$ en $x$, et $\mathscr{F}(x)$ le $\mathbb{C}$-espace vectoriel de dimension finie $\mathscr{F}_{x} / \mathfrak{m}_{x} \mathscr{F}_{x}$ où $\mathfrak{m}_{x}$ est l'idéal maximal de $\mathscr{O}_{X, x}$. Le lemme de Nakayama implique que $\operatorname{dim}_{\mathbb{C}} \mathscr{F}(x)$ est le plus petit entier $r$ tel qu'il existe un voisinage $U$ de $x$ et une surjection $\mathbb{O}_{U}^{r} \rightarrow \mathscr{F}_{\mid U}$.

6.1.2. Lemme. Soit $\left(X, \mathrm{O}_{X}\right)$ un espace analytique de Stein, et soit $\mathscr{F}_{F}$ un $\mathrm{O}_{X}$-module cohérent. Pour que le $\mathscr{H}(X)$-module $H^{0}(X, \mathscr{F})$ soit de type fini, il faut et il suffit que la fonction $x \mapsto \operatorname{dim}_{\mathbb{C}} \mathscr{F}(x)$ soit bornée sur $X$.

Démonstration. La nécessité est évidente : si $H^{0}(X, \mathscr{F})$ est engendré par $r$ éléments, alors la fonction en question est majorée par $r$.

Pour montrer la suffisance, on procède par récurrence sur la dimension $d$ du support de $\mathscr{F}$, avec la convention $\operatorname{dim}(\varnothing)=-1$. Si $d=-1$ alors $\mathscr{F}$ est nul et il n'y a rien à démontrer.

Soit $r$ un majorant de la fonction $x \mapsto \operatorname{dim}_{\mathbb{C}} \mathscr{F}(x)$. Comme l'ensemble des composantes irréductibles de $\operatorname{Supp}(\mathscr{F})$ est localement fini, il existe un sous-espace fermé discret $Z \subset X$ qui rencontre toutes ces composantes. Pour chaque $z \in Z$, soit $\left(s_{1, z}, \ldots, s_{r, z}\right)$ une famille génératrice à $r$ éléments de $\mathscr{F}(z)$. On obtient donc $r$ sections globales de la restriction de $\mathscr{F}$ à $Z$; comme $X$ est de Stein, elles se relèvent en $r$ sections $s_{1}, \ldots, s_{r}$ de $\mathscr{F}$ sur $X$, définissant un morphisme $\varphi: O_{X}^{r} \rightarrow \mathscr{F}$. Notons $\mathscr{F}_{1}$ le conoyau de $\varphi$, qui est un $O_{X}$-module cohérent. Par construction, $\varphi$ est surjectif au voisinage de $Z$, de sorte que Supp ( $\left.\mathscr{F}_{1}\right)$ ne contient aucune composante de $\operatorname{Supp}(\mathscr{F})$. Donc $\operatorname{dim} \operatorname{Supp}\left(\mathscr{F}_{1}\right)<d$, et par hypothèse de récurrence $H^{0}\left(X, \mathscr{F}_{1}\right)$ est un $\mathscr{H}(X)$-module de type fini (noter que comme $\mathscr{F}_{1}$ est un quotient de $\mathscr{F}$ on a $\operatorname{dim} \mathscr{F}_{1}(x) \leq \operatorname{dim} \mathscr{F}(x)$ pour tout $\left.x \in X\right)$. Comme $X$ est de Stein, la suite exacte $\mathrm{O}_{X}^{r} \rightarrow \mathscr{F}_{F} \rightarrow \mathscr{F}_{1} \rightarrow 0$ donne une suite exacte $\mathscr{H}(X)^{r} \rightarrow H^{0}\left(X, \mathscr{F}_{F}\right) \rightarrow H^{0}\left(X, \mathscr{F}_{1}\right) \rightarrow 0$ de $\mathscr{H}(X)$-modules, qui montre que $H^{0}(X, \mathscr{F})$ est de type fini. (L'argument montre plus précisément qu'il est engendré par $r(d+1)$ éléments). 
6.2. Théorème. Soit $\left(X, O_{X}\right)$ un espace analytique de Stein, irréductible et réduit. Alors l'anneau $\mathscr{H}(X)$ vérifie $(\mathrm{C})$.

Démonstration. Posons $A=\mathscr{H}(X)$, et procédons par récurrence sur $d:=\operatorname{dim} X$. Si $d=0$, alors $A=\mathbb{C}$. Si $d>0$, soient $P \in X$ et $Y \subset X$ comme dans la proposition 6.1. On note $\mathfrak{p}$ l'idéal de $Y$ dans $A$, et $\mathfrak{m}$ l'idéal maximal du point $P$. Appliquons le lemme 3.2 avec $\mathfrak{p}_{1}=\mathfrak{p}$ et $\mathfrak{p}_{2}=\mathfrak{m}$. La proposition 6.1 assure que les conditions (i) et (ii) de 3.2 sont satisfaites (cf. la remarque 3.2.1(2)). De plus $\mathfrak{p}_{1}$ est de type fini d'après 6.1 (iii), et il en est de même de $\mathfrak{p}_{2}$ d'après le lemme 6.1.2. L'anneau $A / \mathfrak{p}_{1}$ s'identifie à $\mathscr{H}(Y)$ donc vérifie $(\mathrm{C})$ par hypothèse de récurrence, et $A / \mathfrak{p}_{2} \cong \mathbb{C}$ vérifie trivialement $(\mathrm{C})$, donc le lemme 3.2 donne bien le résultat voulu.

\section{Références}

[Bosch et al. 1990] S. Bosch, W. Lütkebohmert et M. Raynaud, Néron models, Ergebnisse der Math.

(3) 21, Springer, Berlin, 1990. MR 91i :14034 Zbl 0705.14001

[Bourbaki 1961] N. Bourbaki, Algèbre commutative, Chapitres 3 et 4, Actualités Scientifiques et Industrielles 1293, Hermann, Paris, 1961. MR 30 \#2027

[Bourbaki 1965] N. Bourbaki, Algèbre commutative, Chapitre 7 : Diviseurs, Actualités Scientifiques et Industrielles 1314, Hermann, Paris, 1965. MR 41 \#5339

[Davis et al. 1976] M. Davis, Y. Matijasevič et J. Robinson, "Hilbert's tenth problem : Diophantine equations : positive aspects of a negative solution", pp. 323-378 dans Mathematical developments arising from Hilbert problems (De Kalb, IL, 1974), édité par F. E. Browder, Proc. Sympos. Pure Math. 28, Amer. Math. Soc., Providence, R. I., 1976. MR 55 \#5522 Zbl 0346.02026

[Demeyer 2007] J. Demeyer, Diophantine sets over polynomial rings and Hilbert's Tenth Problem for function fields, Ph.D. thesis, 2007.

[Denef et Lipshitz 1980] J. Denef et L. Lipshitz, "Ultraproducts and approximation in local rings. II”, Math. Ann. 253 :1 (1980), 1-28. MR 82g :13021 Zbl 0426.13010

[Grauert et Remmert 1979] H. Grauert et R. Remmert, Theory of Stein spaces, Grundlehren der math. Wiss. 236, Springer, Berlin, 1979. Reimprimé 2004. MR 82d :32001 Zbl 0433.32007

[Grothendieck 1965] A. Grothendieck, "Éléments de géométrie algébrique, IV : Étude locale des schémas et des morphismes de schémas, II”, Inst. Hautes Études Sci. Publ. Math. 24 (1965), 1231. MR 33 \#7330 Zbl 0135.39701

[Grothendieck 1967] A. Grothendieck, "Éléments de géométrie algébrique, IV : Étude locale des schémas et des morphismes de schémas, IV”, Inst. Hautes Études Sci. Publ. Math. 32 (1967), 1361. MR 39 \#220 Zbl 0153.22301

[Matsumura 1989] H. Matsumura, Commutative ring theory, $2^{\mathrm{e}}$ éd., Cambridge Studies in Advanced Mathematics 8, Cambridge Univ. Press, Cambridge, 1989. MR 90i :13001 Zbl 0666.13002

[Pfister et Popescu 1975] G. Pfister et D. Popescu, "Die strenge Approximationseigenschaft lokaler Ringe”, Invent. Math. 30 :2 (1975), 145-174. MR 52 \#395 Zbl 0293.13011

[Popescu 1986] D. Popescu, "General Néron desingularization and approximation", Nagoya Math. J. 104 (1986), 85-115. MR 88a :14007 Zbl 0592.14014

[Raynaud 1970] M. Raynaud, Anneaux locaux henséliens, Lecture Notes in Mathematics 169, Springer, Berlin, 1970. MR 43 \#3252 Zbl 0203.05102 
[Shlapentokh 1994] A. Shlapentokh, "Diophantine classes of holomorphy rings of global fields", $J$. Algebra 169 :1 (1994), 139-175. MR 95h :12007 Zbl 0810.11073

[Spivakovsky 1994] M. Spivakovsky, "Non-existence of the Artin function for Henselian pairs", Math. Ann. 299 :4 (1994), 727-729. MR 95g :13008 Zbl 0803.13005

[Spivakovsky 1999] M. Spivakovsky, "A new proof of D. Popescu's theorem on smoothing of ring homomorphisms", J. Amer. Math. Soc. 12 :2 (1999), 381-444. MR 99j:13008 Zbl 0919.13009

[Swan 1998] R. G. Swan, "Néron-Popescu desingularization", pp. 135-192 dans Algebra and geometry (Taipei, 1995), Lect. Algebra Geom. 2, Int. Press, Cambridge, MA, 1998. MR 2000h :13006 Zbl 0954.13003

Communicated by Bjorn Poonen

Received 2007-07-30 Revised 2007-09-18 Accepted 2007-10-18

laurent.moret-bailly@univ-rennes1.fr

IRMAR (Institut de Recherche Mathématique de Rennes, UMR 6625 du CNRS), Université de Rennes 1, Campus de Beaulieu, F-35042 Rennes Cedex, France http://perso.univ-rennes1.fr/laurent.moret-bailly/ 


\section{Guidelines for Authors}

Authors may submit manuscripts in PDF format on-line at the Submission page at the ANT website.

Originality. Submission of a manuscript acknowledges that the manuscript is original and and is not, in whole or in part, published or under consideration for publication elsewhere. It is understood also that the manuscript will not be submitted elsewhere while under consideration for publication in this journal.

Language. Articles in ANT are usually in English, but articles written in other languages are welcome.

Required items. A brief abstract of about 150 words or less must be included. It should be self-contained and not make any reference to the bibliography. If the article is not in English, two versions of the abstract must be included, one in the language of the article and one in English. Also required are keywords and subject classifications for the article, and, for each author, postal address, affiliation (if appropriate), and email address.

Format. Authors are encouraged to use $\mathrm{LT}_{\mathrm{E}} \mathrm{X}$ but submissions in other varieties of $\mathrm{T}_{\mathrm{E}} \mathrm{X}$, and exceptionally in other formats, are acceptable. Initial uploads should be in PDF format; after the refereeing process we will ask you to submit all source material.

References. Bibliographical references should be complete, including article titles and page ranges. All references in the bibliography should be cited in the text. The use of $\mathrm{BibT}_{\mathrm{E}} \mathrm{X}$ is preferred but not required. Tags will be converted to the house format, however, for submission you may use the format of your choice. Links will be provided to all literature with known web locations and authors are encouraged to provide their own links in addition to those supplied in the editorial process.

Figures. Figures must be of publication quality. After acceptance, you will need to submit the original source files in vector graphics format for all diagrams in your manuscript: vector EPS or vector PDF files are the most useful.

Most drawing and graphing packages (Mathematica, Adobe Illustrator, Corel Draw, MAT$\mathrm{LAB}$, etc.) allow the user to save files in one of these formats. Make sure that what you are saving is vector graphics and not a bitmap. If you need help, please write to graphics@mathscipub.org with details about how your graphics were generated.

White Space. Forced line breaks or page breaks should not be inserted in the document. There is no point in your trying to optimize line and page breaks in the original manuscript. The manuscript will be reformatted to use the journal's preferred fonts and layout.

Proofs. Page proofs will be made available to authors (or to the designated corresponding author) at a Web site in PDF format. Failure to acknowledge the receipt of proofs or to return corrections within the requested deadline may cause publication to be postponed. 


\section{Algebra \& Number Theory}

Volume $1 \quad$ No. $3 \quad 2007$

Contracted ideals and the Gröbner fan of the rational normal curve

Aldo Conca, Emanuela De Negri and Maria Evelina Rossi

Swan conductors for $p$-adic differential modules, I: A local construction

269 KIRAN S. KEDLAYA

$L_{\infty}$ structures on mapping cones

301

DOMENiCO FIORENZA AND MARCo MANETti

Sur la définissabilité existentielle de la non-nullité dans les anneaux

LAURENT MORET-BAILLY 